\title{
Electrical Characterization of Silicon Strip Sensors for the ATLAS ITk at the HL-LHC with Extended Investigations of Sensor Properties
}

by

\section{Ezekiel Staats}

A thesis submitted to the Faculty of Graduate and Postdoctoral Affairs in partial fulfillment of the requirements for the degree of

\author{
Master of Science \\ in \\ Physics \\ Department of Physics \\ Carleton University \\ Ottawa, Ontario \\ December 2020 \\ (c) 2020 \\ Ezekiel Staats
}




\begin{abstract}
Silicon $n^{+}-i n-p$ microstrip sensors have been developed for the ATLAS Inner Tracker (ITk). This is part of the phase-II upgrade programme for the High Luminosity Large Hadron Collider which will see a sevenfold increase in peak instantaneous luminosity. Prototype and preproduction generations of strip sensors are designed by the ITk Strip Sensor Collaboration and produced by Hamamatsu Photonics.

The sensors undergo a variety of electrical and physical tests for Quality Control (QC) which are essential to verify compliance with specifications, monitor processing consistency, and predict the overall performance of the ITk. QC procedures are detailed and results from prototype sensors are given. A selection of results beyond the scope of QC are also presented. Topics include the effects of proton radiation, anomalous loss of strip isolation, and bias dependent changes in surface oxide charges. These studies are aimed at informing predictions of long term sensor performance.
\end{abstract}


To my dear, Tiffany 


\section{Acknowledgements}

There are a great many people who have both directly and indirectly contributed to my advancement in physics, and ultimately, the completion of this thesis. The foremost of these is my project supervisor, Dr. Thomas Koffas, who has tirelessly guided me and continually provoked me to hard work.

A debt of thanks is also owed to the senior members of the Carleton ITk group, Dr. Dag Gillberg, Dr. John Keller, and Dr. Christoph Klein for their ongoing support and expertise. I also would like to express gratitude to my fellow students in Carleton's ATLAS group whose camaraderie has continually lifted my spirits throughout this degree.

I wish to thank Dr. Bart Hommels at the University of Cambridge, England, for devoting a week of time toward training me in sensor QC procedures and for the gracious tour of his lab.

Lastly, I must express my deepest thanks to my wife, mother, and siblings who have offered me unbounded support and counsel throughout my life's endeavours. 


\section{Contents}

$\begin{array}{lll}\text { Abstract } & \text { ii }\end{array}$

Acknowledgements $\quad$ iv

Contents $\quad$ v

List of Tables

$\begin{array}{ll}\text { List of Figures } & \text { ix }\end{array}$

List of Acronyms

Statement of Author's Contributions xiii

1 Introduction $\quad 1$

1.1 The LHC and High Luminosity Upgrade . . . . . . . . . . . . . . . . . . . . . . . . . . . . . . . . .

1.1.1 The HL-LHC Upgrade . . . . . . . . . . . . . . . . . . . . . . . . 3

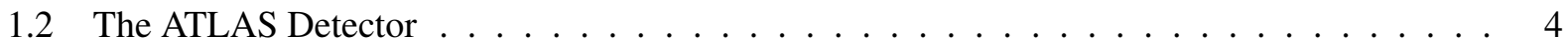

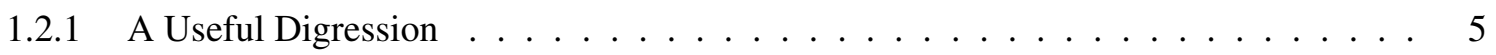

1.2 .2 The Inner Detector $\ldots \ldots \ldots \ldots \ldots$

1.3 The ITk Upgrade $\ldots \ldots \ldots \ldots$

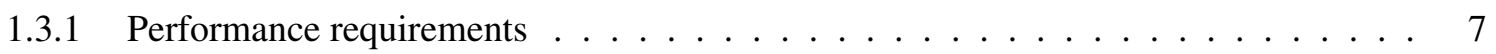

1.4 ITk Physical Layout $\ldots \ldots \ldots \ldots \ldots$

1.4 .1 Strip Detector Layout . . . . . . . . . . . . . . . . . . . . . . 9

1.4 .2 Pixel Detector Layout $\ldots \ldots \ldots \ldots \ldots$

1.4.3 Radiation Environment . . . . . . . . . . . . . . . . . . . . . 11

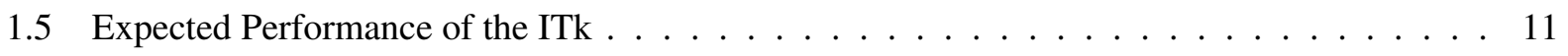

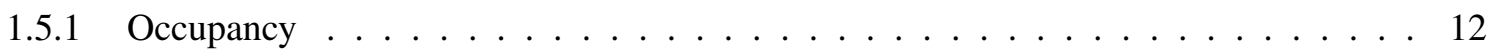

1.5.2 Track Reconstruction . . . . . . . . . . . . . . . . . . . . . 12

1.5.3 Tracking Efficiencies and Fake Rates . . . . . . . . . . . . . . 13 
1.5.4 Track Parameter Resolution ． . . . . . . . . . . . . . . . . . . . . . 14

1.5.5 Primary Vertex Reconstruction . . . . . . . . . . . . . . . . . . . . . 16

2 Semiconductor Theory $\quad 18$

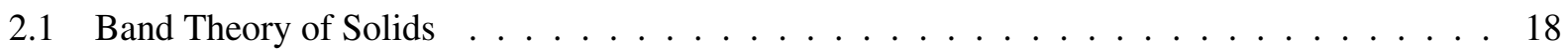

2.2 N-type and P-type Semiconductors . . . . . . . . . . . . . . . . . . . . . . . . 19

2.3 The P-N Junction and Biasing . . . . . . . . . . . . . . . . . . . 21

2.3.1 General Characteristics . . . . . . . . . . . . . . . . . . . . 21

2.3.2 The Step Junction and Depletion Width . . . . . . . . . . . . . . . . . 22

2.3.3 Step Junction Capacitance Characteristics . . . . . . . . . . . . . . . . . . . 25

2.4 Principle of Operation for Silicon Strip Sensors ～. . . . . . . . . . . . . . . . . 26

2.5 Effects of Radiation . . . . . . . . . . . . . . . . . . . . . . . . 27

2.5 .1 Bulk Damage . . . . . . . . . . . . . . . . . . . 27

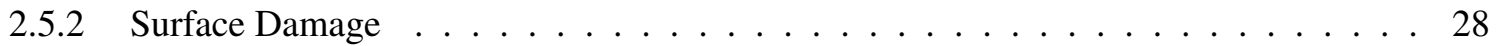

3 Sensor Testing $\quad 29$

3.1 The ITk Sensor . . . . . . . . . . . . . . . . . . . . . . . . . . . . . . . . . 29

$3.1 .1 \quad$ Barrel Sensor Layout $\ldots \ldots \ldots \ldots$

3.1 .2 End-cap Sensor Layout . . . . . . . . . . . . . . . . . . . . 31

3.1 .3 Test Devices . . . . . . . . . . . . . . . . . . . . . 33

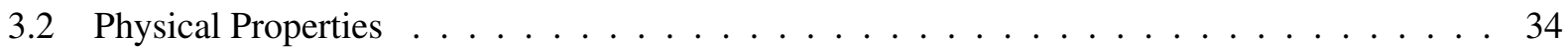

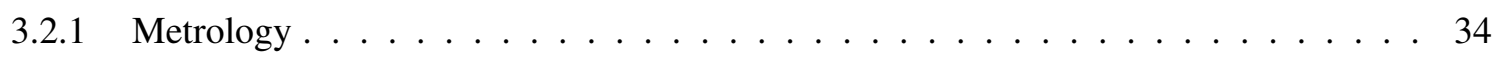

3.2 .2 Image Capture . . . . . . . . . . . . . . . . . . 35

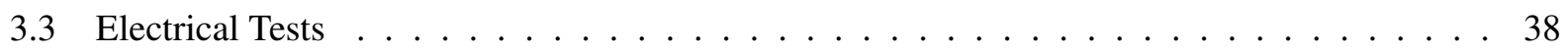

3.3.1 IV/CV Characteristics . . . . . . . . . . . . . . . . . . 39

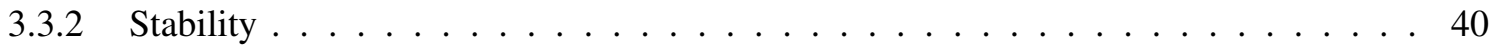

3.3 .3 Full Strip Test . . . . . . . . . . . . . . . . . . . . . 40

3.3 .4 Interstrip Resistance . . . . . . . . . . . . . . . . . . . . . . . 41

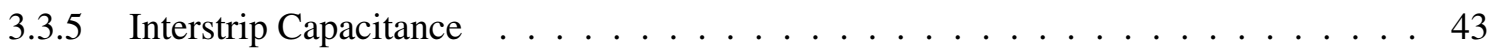

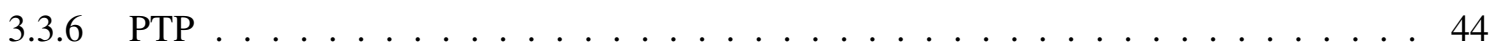

4 Results for Prototype Sensors $\quad 45$

4.1 Physical Properties . . . . . . . . . . . . . . . . . . . 45

4.1 .1 Metrology Results . . . . . . . . . . . . . . . . . . . . . 45

4.2 Electrical Tests . . . . . . . . . . . . . . . . . . 47

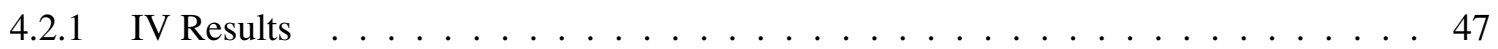

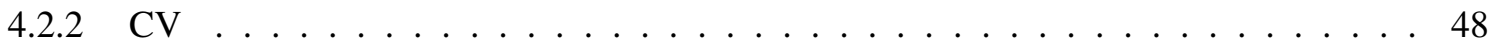

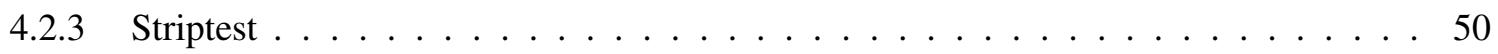


4.2.4 Interstrip Resistance and Capacitance $\ldots \ldots \ldots \ldots \ldots \ldots$

5 Further Investigations of Sensor Properties 56

5.1 Performance after Irradiation $\ldots \ldots \ldots \ldots$

5.1 .1 Test Setup for Irradiated Minis . . . . . . . . . . . . . . . . 56

5.1 .2 Current-Voltage Response of Irradiated Minis . . . . . . . . . . . . . . . . . 57

5.2 Case Study: Anomalous Loss of Interstrip Isolation $\ldots \ldots \ldots$

5.2 .1 Initial Sensor Performance . . . . . . . . . . . . . . . . . . . 59

5.2 .2 Sensor Degradation . . . . . . . . . . . . . . . . . . . . 60

5.2 .3 Partial Sensor Recovery . . . . . . . . . . . . . . . . . . 60

5.2 .4 Examining Strip Isolation $\ldots \ldots \ldots \ldots$. . . . . . . . . . . 61

5.3 Properties of the Surface Oxide . . . . . . . . . . . . . . . . . . . 62

5.3 .1 Characteristics of MOS Capacitors . . . . . . . . . . . . . . 62

5.3.2 MOS Capacitors of ATLAS18R0 Sensors . . . . . . . . . . . . . . 64

5.3 .3 Field Enhanced Charge Injection . . . . . . . . . . . . . . . 65

6 Summary and Outlook $\quad 68$

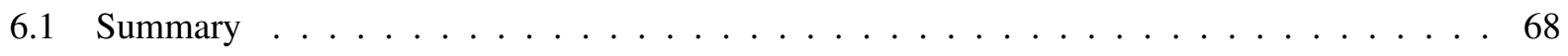

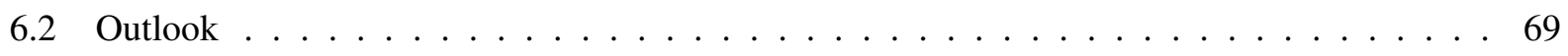

$\begin{array}{ll}\text { Bibliography } & \mathbf{7 0}\end{array}$ 


\section{List of Tables}

1.1 Target track finding efficiencies. $[6] \ldots \ldots \ldots \ldots \ldots \ldots$

1.2 Layout parameters for barrel section of strip detector. $[6] \ldots \ldots$. . . . . . . . . . 9

1.3 Layout parameters for encap section of strip detector. [6] . . . . . . . . . . . . . . 10

1.4 Expected fluence and dose distributions in ITk. [9],[10],[11] . . . . . . . . . . . 11

3.1 Sensor specifications common to all sensor layouts. Unlisted irradiation specifications are the same as the pre-irradiation specifications. . . . . . . . . . . . . . . . 30

3.2 Outline of QA test devices. The test devices are all laid out on one of four halfmoons accompanying each main sensor. The ATLAS test chip includes all structures following this entry. Corresponding labels as given in Figure 3.3 (left) are also given in brackets under the "Test Device" column. . . . . . . . . . . . . . . . . . . . . . . . 33

3.3 Outline of specifications for the metrology setup at the Carleton site . . . . . . . . . . . . 34

3.4 Outline of specifications for the image capturing setup at the Carleton site. Descriptions of working parameters for the robotic arm, camera, and lens are given. The four joints of the robotic arm are referred to as $\mathrm{J} 1-\mathrm{J} 4$. $\mathrm{J} 1$ and $\mathrm{J} 2$ translate in $x y, \mathrm{~J} 3$ translates in $z$, and $\mathrm{J} 4$

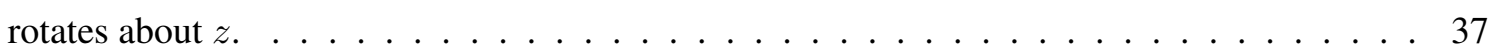

3.5 Parameters for Carleton site image capture setup as shown in Figure 3.6. . . . . . . . . . 37 


\section{List of Figures}

1.1 A schematic of the LHC complex. The four main detectors are shown along the main ring: CMS, LHCb, ALICE, and ATLAS. Various beam lines (coloured arrows denote types of particles), accelerators, and experiments are also shown. Protons are generated in Linac 2 and pass through the booster, Proton Synchrotron (PS), and the Super Proton Synchrotron (SPS) before reaching the main ring. $[1] \ldots \ldots \ldots \ldots \ldots \ldots$

1.2 HL-LHC upgrade schedule. $[2] \ldots \ldots \ldots \ldots$

1.3 Schematic of ATLAS revealing "onion-geometry" of the sub-detector systems. [3] _ . . . 4

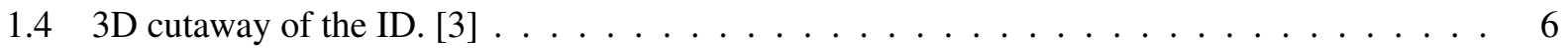

1.5 2D schematic layout of ITk. The layout is mirrored across the $R$-axis. The strip and pixel sub-detector systems are shown in blue and red, respectively. In both cases, the darker shaded colour identifies the endcap region. [6] . . . . . . . . . . . . . . . . . 8

$1.63-\mathrm{D}$ visualization of the ITk detector. The pixel detector is shown in red and dark blue (barrel and endcap) while the strip detector elements are given a light blue green colour scheme. [6] 9

1.7 Channel occupancy from minimum bias events with $\langle\mu\rangle=200$ for the strip detector. Barrel (left) and endcap (right) regions are shown.[6] . . . . . . . . . . . . . . . . 12

1.8 Track reconstruction efficiency as a function of $\eta$ for three scenarios (top left, top right, bottom left). The final plot (bottom right) indicates that efficiency is nearly independent of pileup in all $\eta$ regions.[6] . . . . . . . . . . . . . . . . . . . . . . . 13

1.9 Ratio of reconstructed tracks to number of charged particles plotted against $\mu$. [6] . . . . 14

1.10 Resolutions shown for each of the five track parameters as a function of $\eta$. Comparisons to Run 2 data are also shown. [6] . . . . . . . . . . . . . . . . . . . 15

1.11 Distribution of primary reconstructed vertices per event in $t \bar{t}$ sample with $\mu=200$. [6] . . . 17

2.1 Schematic representation of energy band structure of solids. A and B demonstrate two configurations leading to conducting materials. $\mathrm{C}$ demonstrates a configuration leading to insulators or semiconductors depending on the size of the energy gap $\left(\mathrm{E}_{g}\right)$. [15] . . . . . . 19

2.2 Examples of $\mathrm{n}$ and $\mathrm{p}$-type semiconductors (top and bottom, respectively). The band structure for the n-type material is also shown with the donor level just below the conduction band

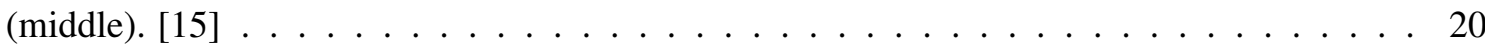


2.3 A p-n junction where electron and hole concentrations are shown schematically. Left behind dopant ions are also depicted in the depletion region. [17] . . . . . . . . . . . . . . . 22

2.4 Schematic charge density and electric field strength vs distance in a p-n step junction. . . . . 23

2.5 Schematic cross section of a $\mathrm{p}^{+}$-in-n silicon strip sensor. ITk sensors operate using the same basic principles but the types are reversed.[18] . . . . . . . . . . . . . . 27

3.1 Example of a cross section through the interstrip region of a sensor. The strip pitch given is that of barrel sensors. The diagram is to scale in the horizontal direction only. All units are

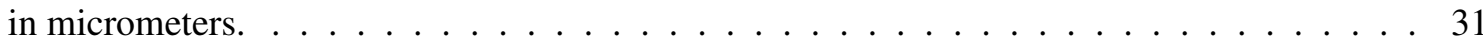

3.2 (Left) Demonstration of the stereo annulus geometry. (Right) The hashed lines define the centre of the sensor, $\mathrm{O}_{\mathrm{w}}$. The solid blue lines indicate the sensor's radial position in the global coordinates and on a petal. [6] . . . . . . . . . . . . . . . 32

3.3 (Left) Example wafer layout of a barrel sensor demonstrating the halfmoons at the sensor periphery. Dicing lanes are denoted by yellow lines. The four ATLAS test chips are highlighted in red. (Right) Schematic of the ATLAS test chip. . . . . . . . . . . . . . 32

3.4 Schematic representation demonstrating the basic principle of the light-section method. In this example, $\Delta \mathrm{h}$ is determined through basic trigonometry with the known angle of transmittance and the measured displacement $\Delta \mathrm{d} \ldots \ldots \ldots \ldots \ldots \ldots$

3.5 Rendering of the image capture setup used at the Carleton site. The robotic arm moves the sensor below the fixed camera. Micrometer adjustable stages are used to mount the camera to the support bar. This ensures the camera can be properly aligned and parallel to the sensor. 36

3.6 Layout of all image capture maps. Also detailed in Table 3.5 . . . . . . . . . . . . . . . 38

3.7 Circuit diagrams for the IV (top left) and CV (top right) test [23]. (Bottom left) Example IVs of three sensors demonstrating three different behaviours: early and immediate breakdown, late and gradual breakdown, no breakdown. (Bottom right) Example CVs of the same three sensors. One sensor reaches full depletion much later than the other two. QC limits are given by hashed lines. . . . . . . . . . . . . . . . . . . . . . . 40

3.8 Circuit diagram for the full strip test including the optional switch matrix. [23] $\ldots \ldots$. . 41

3.9 Circuit diagram for the interstrip resistance. Adapted from [23] . . . . . . . . . . . . . 42

3.10 (Left) The first loop corresponding to equation 3.2. (Right) The loop corresponding to equation 3.3. Adapted from $[23] \ldots \ldots \ldots \ldots \ldots \ldots$

3.11 Circuit diagram for interstrip capacitance $[23] \ldots \ldots \ldots \ldots$

3.12 Circuit diagram for PTP $[23] \ldots \ldots \ldots \ldots \ldots \ldots$

4.1 Measured sensor bow and thickness for eight batch 3 ATLAS12 R0 sensors. . . . . . . . . 46

4.2 IV curves from three groupings of sensors: ATLAS12 R0 (top left), ATLAS17 LS (top right), ATLAS18 R0 (bottom left). . . . . . . . . . . . . . . . . 47 
4.3 CV curves from three groupings of sensors: Batch 2 ATLAS12 R0 (top left), ATLAS17 LS (top right), Batch 3 ATLAS12 R0 (center). (Bottom left) A histogram of the effective doping concentration obtained for all sensors. (Bottom right) A histogram of the depletion depth at full depletion for all sensors. . . . . . . . . . . . . . . . . . . . . . . . 49

4.4 (Top) Flowchart of strip defect classification. (Bottom) Images of specific defects which may arise in the ITk strip sensor manufacturing process. [28] . . . . . . . . . . . 50

4.5 Striptest results from an ATLAS17 LS barrel sensor, W020 . . . . . . . . . . . . . . 52

4.6 Striptest results from an ATLAS12 R0 endcap sensor, W194 . . . . . . . . . . . . . . 53

4.7 Striptest results from an ATLAS12 R0 endcap sensor, W214 . . . . . . . . . . . . . . 54

4.8 Interstrip resistance and capacitance measured against bias voltage in ATLAS17 barrel sensors. 55

5.1 Schematic side view of the "cooling jig" setup used to test the irradiated minis. Coolant is refrigerated and pumped through the copper chuck while thermoelectric peltiers draw heat out of the aluminum enclosure and deposit that heat into the copper. Stable environments of $-20^{\circ} \mathrm{C}$ and $<35 \%$ relative humidity are achievable with this setup. . . . . . . . . . 57

5.2 Current voltage response of 14 proton irradiated minis. The minis are irradiated to fluences $10^{14}$ (circle), $5 \times 10^{14}$ (triangle), $10^{15}$ (diamond), $2 \times 10^{15}$ (square), and $10^{16} n_{e q} / \mathrm{cm}^{2}$ (cross). 58

5.3 Initial sensor bulk characteristics on intake. . . . . . . . . . . . . . . . . . . 59

5.4 Degraded sensor performance in the IV and bias resistance strip-level test. . . . . . . . . 60

5.5 Partial recovery of sensor performance in the IV and bias resistance strip-level test. . . . . . 61

5.6 Measurements of the interstrip resistance at two different locations in segment 1 of sensor W020. (Left) A well behaved region and (right) a region with loss of isolation. Note the discrepancy in the vertical axis between the two plots. Error bars in the right graph are too small to display. The error is small in the case of low isolation because larger voltages are induced in the central strip. . . . . . . . . . . . . . . . . . . . 62

5.7 Energy band representation of the flatband condition. (A) The materials on their own with different fermi levels and associated work functions. (B) The materials in a MOS capacitor with no voltage applied to the gate. The energy bands of the semiconductor bend down. (C) The band diagram of the materials when $V_{F B}$ is applied to the gate and the flatband is restored in the semi-conductor. $[16] \ldots \ldots \ldots \ldots$

5.8 Circuit diagram of the test setup used to obtain the CV of the ATLAS18 MOS capacitor. . . 64

5.9 MOS Capacitors. . . . . . . . . . . . . . . . . . . . 66

5.10 Field enhanced charge injection of a MOS capacitor for three field strengths. . . . . . . . 67 


\section{List of Acronyms}

AC - Alternating Current

ASIC - Application Specific Integrated Circuit

ATLAS - A Toroidal LHC ApparatuS

CERN - (French) Conseil Européen pour la Recherche Nucléaire, (English) European Council for Nuclear Research

CV - Capacitance vs. Voltage (for MOS device) or $1 / C^{2}$ vs. Voltage (for main sensor)

DC - Direct Current

DUT - Device Under Test

(HL)-LHC - (High Luminosity) Large Hadron Collider

ID - Inner Detector

ITk - Inner Tracker

IV - Current vs. Voltage

LCR (meter) - Impedance Capacitance Resistance (meter)

LS (main sensor) - Long Strip barrel (main sensor)

LSM - Least Square Method

MIP - Minimum Ionizing Particle

MOS - Metal Oxide Semiconductor

PTP - Punch Through Protection

QA - Quality Assurance

QC - Quality Control

R0-R5 (main sensor) - $0^{\text {th }}$ to $5^{\text {th }}$ Row endcap (main sensor)

RC (circuit) - Resistance Capacitance (circuit)

RH - Relative Humidity

RMS - Root Mean Square

RT - Room Temperature

SCT - SemiConductor Tracker

SMU - Source and Measure Unit

SS (main sensor) - Short Strip barrel (main sensor)

TRT - Transition Radiation Tracker 


\section{Statement of Author's Contributions}

The first chapter of this manuscript contains no original content but provides relevant background of the High Luminosity upgrade to the Large Hadron Collider and the associated Inner Tracker upgrade for ATLAS. Similarly, the second chapter is not original as semiconductor theory is well established, however, it is essential to provide the framework for the reader to understand the basic principle of operation of the silicon strip sensors.

The contents of chapter three, sensor design and QC test development, are a result of the broader ITk strip sensor community in which the author has had direct involvement. The analysis of results given in chapter four are all original work with the data acquisition largely performed by the author but, in some cases, carried out by other Carleton practitioners. The author was intimately involved in the development of the the image capture and metrology systems. These were completely established from non-existent to working setups throughout the duration of this Master's work. Similarly, this work also resulted in the crucial development of the full strip test as implemented with the semi-automatic probe station and switch matrix.

Finally, the discussions and evaluation of sensor properties in chapter five are completely original with the exception of MOS device theory presented in the final section of the chapter. The author has also contributed to several publications which present more results of QC testing of prototype sensors [26], a characterization of sensor surface properties after irradiation [21], a humidity sensitivity of sensors [31], and strip sensor module performance [30]. In these publications, the author's contributions may include QC and QA measurements directly made by the author (which are included in the publication). The contributions may also include confirmation of the sensor properties described in those publications through the measurement of other samples not presented. 


\section{Chapter 1}

\section{Introduction}

A constant pursuit of unanswered questions is the impetus of the natural sciences. Fundamental questions such as "How did the universe come to be?" and "What are its main constituents?" bolster the broad landscape of the unknown. It has been just over 120 years since the discovery of the first subatomic particle, the electron in 1897. The continued success of particle physics over this time period has resulted in the Standard Model (SM), a description of all known particles and their interactions. SM physics provides a framework wherein all fermionic and bosonic matter in the universe can be understood. Furthermore, SM predictions have been tested and confirmed with great precision, up to and including the discovery of the Higgs Boson in 2012. However, despite the great success of SM physics, more still remains waiting to be uncovered. What is the supposed "dark sector" which accounts for over $80 \%$ of matter in the universe? Are there any interactions, so-called portals, between the dark and SM sectors? If there are, can we detect them? Is there yet more to be discovered about SM processes?

The new ATLAS Inner Tracker (ITk) at the Large Hadron Collider (LHC) of CERN seeks to help provide answers to these questions. This upgraded central tracking system is required in order for the detector to continue providing meaningful physics data as the LHC enters a new phase of high luminosity operation. The LHC already produces the highest attained energies and is being upgraded to further its physics reach. During this phase of operation which is already an extension to the project lifetime, ten times more physics data will be collected than that which was initially planned for the collider. This will undoubtedly push the boundary of sensitivity to all interactions within the LHC keeping it at the forefront of high energy physics for years to come.

The ITk's silicon strip sensors are the topic of this Master's thesis. To be particular, these sensors belong to the outer sub-system, the strip detector, of the ITk. Along with their pixel sensor counterparts, they are the essential, signal producing, detecting elements of the ITk. This chapter begins with an overview of the LHC, its impending high luminosity upgrades and the ATLAS detector (Sections 1.1 and 1.2). The ITk upgrade and specific ITk layout are explored next along with associated physics benchmarks (Sections 1.3, 1.4 and 1.5). The following chapters provide more detail into semiconductor theory (Chapter 2), electrical testing of ITk sensors for quality control (Chapter 3), sensor test results of prototype sensors (Chapter 4), 
and an extended study of sensor properties beyond the scope of quality control (Chapter 5). The final chapter provides a summary with an outlook of the ITk project and future studies (Chapter 6).

\subsection{The LHC and High Luminosity Upgrade}

The Large Hadron Collider (LHC) sits at the Swiss-French border as the largest, most energetic particle collider in the world. At its current design luminosity of $\mathscr{L}=10^{34} \mathrm{~cm}^{-2} \mathrm{~s}^{-1}$, the LHC collides bunches of protons (p) in 25ns intervals (bunch-crossings) achieving centre of mass energies up to $14 \mathrm{TeV}$. In an alternate mode of operation, heavy ions, namely lead $(\mathrm{Pb})$ nuclei, can also be collided in the LHC with a design luminosity of $\mathscr{L}=10^{37} \mathrm{~cm}^{-2} \mathrm{~s}^{-1}$ and $5.5 \mathrm{TeV}$ per $\mathrm{Pb}-\mathrm{Pb}$ pair. The LHC accelerator complex is shown in Figure 1.1.

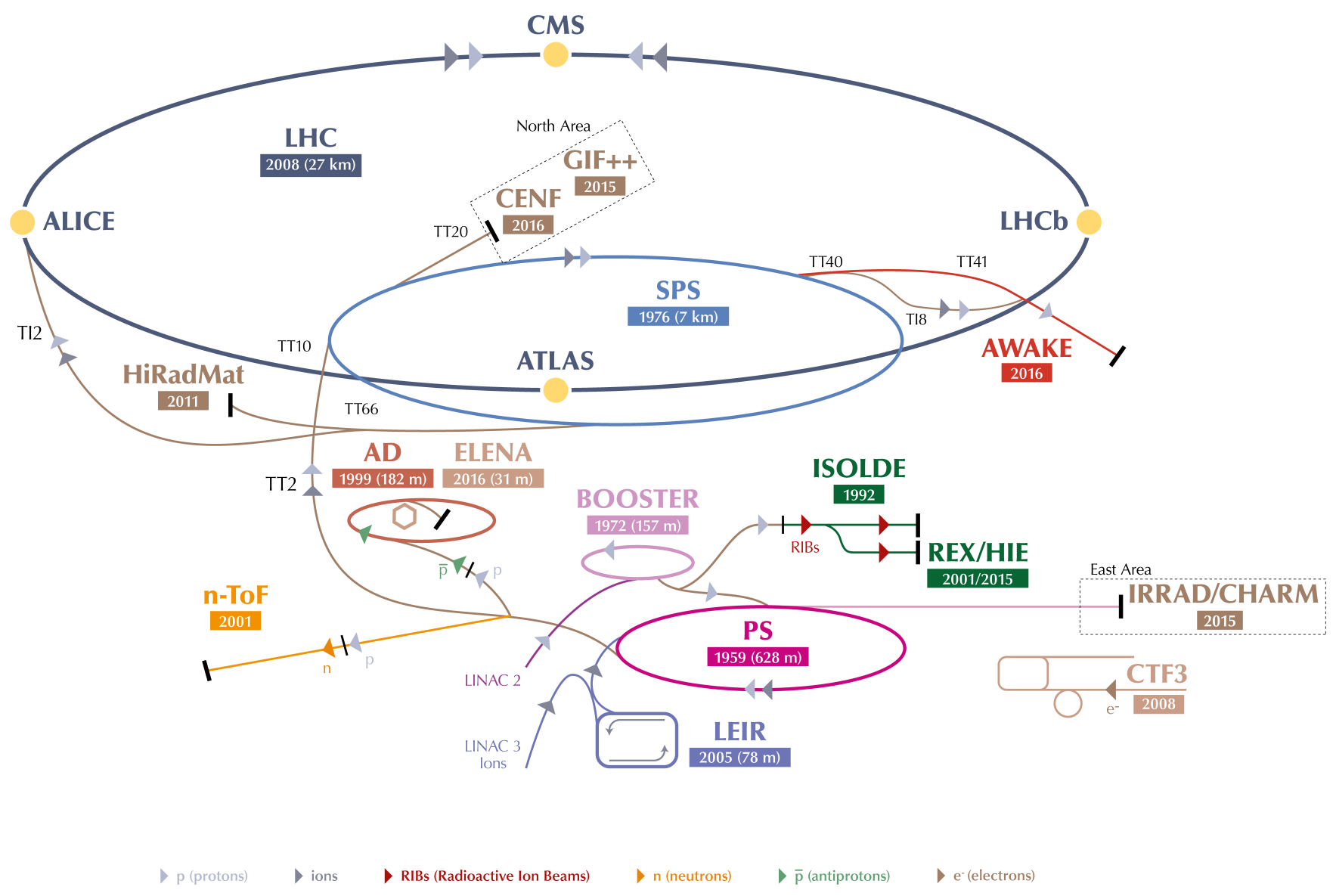

Figure 1.1: A schematic of the LHC complex. The four main detectors are shown along the main ring: CMS, LHCb, ALICE, and ATLAS. Various beam lines (coloured arrows denote types of particles), accelerators, and experiments are also shown. Protons are generated in Linac 2 and pass through the booster, Proton Synchrotron (PS), and the Super Proton Synchrotron (SPS) before reaching the main ring. [1] 
Four detectors are positioned on the main ring, each with unique physics goals. $\mathrm{Pb}-\mathrm{Pb}$ and $\mathrm{p}-\mathrm{p}$ interactions are probed using the two general purpose detectors, A Toroidal LHC ApparatuS (ATLAS) and Compact Muon Solenoid (CMS). A forward detector, the Large Hadron Collider beauty (LHCb), has its main focus on studying bottom-quark and charm-quark physics. Lastly, quark-gluon plasma through $\mathrm{Pb}$ collisions is the main emphasis of A Large Ion Collider Experiment (ALICE).

\subsubsection{The HL-LHC Upgrade}

Energy and statistics are the primary considerations for particle physics experiments, including those at the LHC. Energy defines the type of particle which can be created in a given interaction through the energymass equivalence relation. Heavier particles are rarer and shorter lived and will decay promptly into lighter, more stable daughter particles. Furthermore, the quantum mechanical nature of subatomic particles and their interactions is inherently stochastic. Thus, the potential to observe new physics is limited by both the energy of an interaction and the number of interactions produced at that energy. In collider physics, the number of interactions produced is dictated by the collision rate and period over which the experiment is carried out.

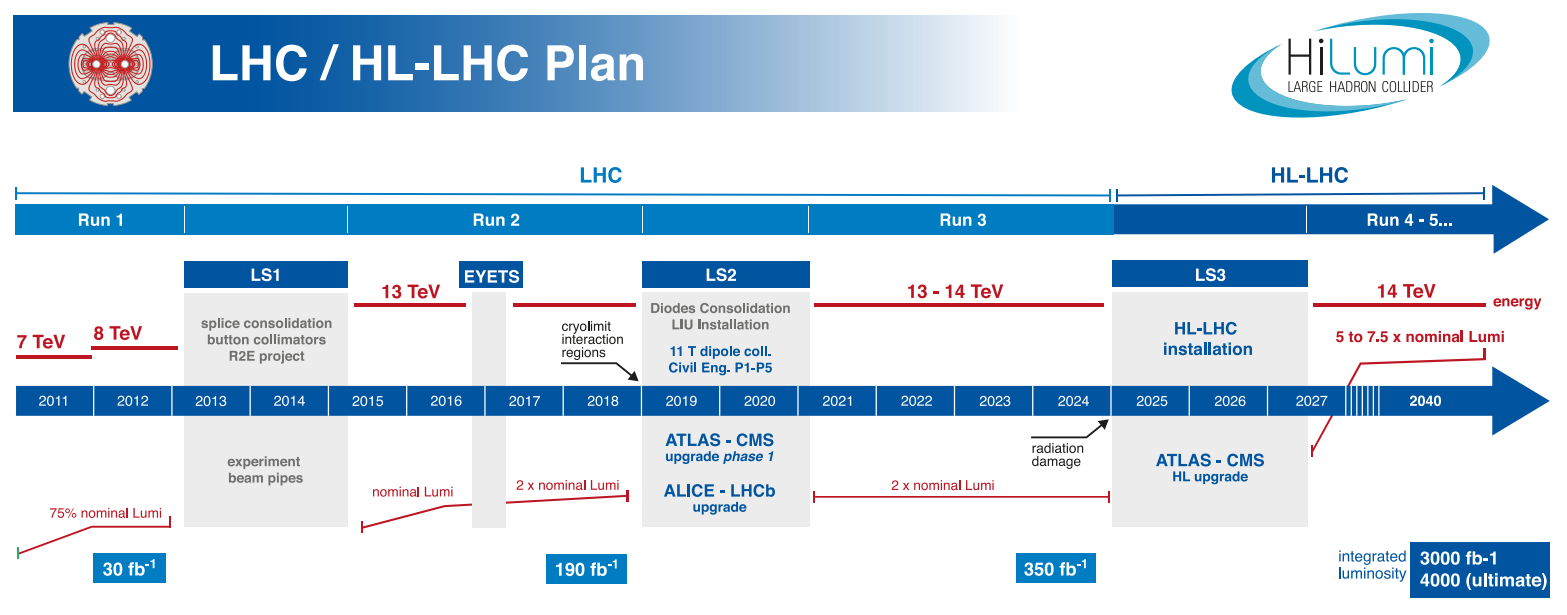

Figure 1.2: HL-LHC upgrade schedule. [2]

In the next 6-7 years, the LHC will be upgraded to the High Luminosity LHC (HL-LHC). While interaction energy was the LHC's main motivation, the HL-LHC is focussed on increasing statistics through increased instantaneous luminosity (hence the name). The operation and upgrade schedule, beginning in 2011, for the LHC is shown in Figure 1.2. Multiple runs are separated by Long Shutdowns ("LS" in the figure) allowing for accelerator and detector maintenance as well as upgrades. The first run had the main goal of discovering the Higgs Boson and was conducted at energies of $7 \mathrm{TeV}$ and $8 \mathrm{TeV}$. The first Long Shutdown (LS1) saw extensive maintenance to the entire LHC complex. The emphasis was on magnet issues 
which had already cost almost two years of operation in 2008-2010. After LS1, during the second run, the centre of mass energy was increased to $13 \mathrm{TeV}$ and the LHC's design luminosity was achieved. The LHC is currently in the middle of LS2 where, among other improvements, the ALICE and LHCb experiments are receiving major upgrades. Final preparations for HL-LHC operation will begin during LS3.

Besides a significant performance upgrade of the accelerator, several major detector components of ATLAS and CMS will be replaced. The following section focusses on ATLAS. As the larger detector system into which the ITk will be installed, it is a minor but necessary stop before converging on the ITk and its strip sensors.

\subsection{The ATLAS Detector}

ATLAS is a multipurpose detector which is essentially a large cylinder concentric to the LHC beam line. Weighing over $7000 \mathrm{t}$ and being $44 \mathrm{~m}$ long with a $25 \mathrm{~m}$ diameter, it stands as a scientific and engineering marvel. It is comprised of multiple sub-detector systems in a sort of onion-geometry where each system encases the next. The Inner Detector (ID), which will be further discussed in the following subsection, sits nearest to the beam line as the charged particle tracker. Surrounding the ID is the two-part calorimeter system, a liquid Argon (LAr) electromagnetic sampling calorimeter and a hadronic calorimeter. The muon tracking system makes up the outermost layer.

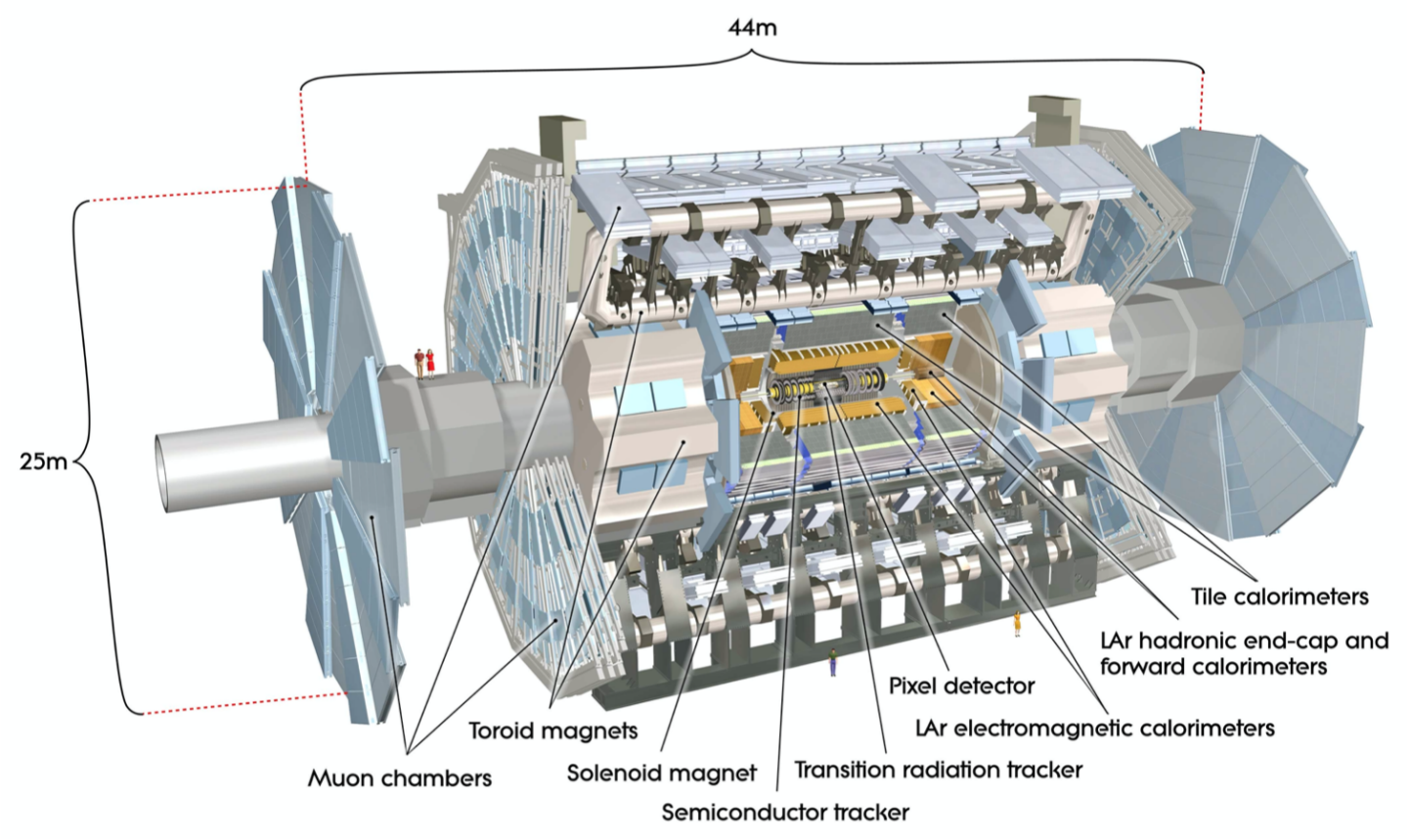

Figure 1.3: Schematic of ATLAS revealing "onion-geometry" of the sub-detector systems. [3] 
The barrel and endcap layout employed by ATLAS (see Figure 1.3) is natural for detectors in collider experiments. The barrel sections surround the beam line, centred at the interaction point and are designed to detect particles which have high transverse momentum. Forward (and back) scattered particles are detected in endcaps which are discs in the transverse plane perpendicular to the beam.

End-of-lifetime performance is a primary concern for ATLAS in the HL-LHC phase of operation. The higher intensity collisions will produce nearly 10 times as many interactions within the detectors. Prolonged operation while maintaining the necessary performance standards is not an easy task in this dense radiation environment. Being closest to the beam line, the ITk will be exposed to higher doses of destructive radiation than any of the other sub-detector systems.

\subsubsection{A Useful Digression}

At this point, before going into further detail on the ID, it is necessary to conduct a short discussion centred around useful parameters used to describe various aspects of a collider experiment.

The first topic introduces a convenient set of coordinates for describing both the physical layout of a detector and the particles travelling through it. The z-axis is defined to coincide with the beam axis. The plane perpendicular to this axis, that is the transverse plane, is chosen so that the $\mathrm{x}$-axis points toward the centre of the LHC ring. The $\mathrm{y}$-axis is defined as pointing upwards vertically. Rather then use these $\mathrm{x}, \mathrm{y}, \mathrm{z}$ coordinates directly, it is helpful to define the azimuthal angle $\phi$, distance from the beam line $R=\sqrt{x^{2}+y^{2}}$, and a polar angle $\theta$. The convenience of this coordinate system can be furthered by the introduction of pseudorapidity $\eta$. This quantity is defined as $\eta=-\ln [\tan (\theta / 2)]$ and approximates rapidity $\left(y=1 / 2 \cdot \ln \left[\left(E-p_{z}\right) /\left(E+p_{z}\right)\right]\right.$ where $E$ and $p_{z}$ are the energy and z-component of the particle's four-momentum, respectively) in the limit where the particle is travelling close to the speed of light, or equivalently, has negligible mass. Rapidity has the advantage of being additive under Lorentz transformations. [4]

At each bunch crossing a number of interesting, hard-scatter collisions occur. This is termed "pileup" and given the character $\mu$. As one might guess, pileup depends on instantaneous luminosity since a more tightly packed intersection of two bunches of protons will produce more scattering events. Pileup plays a significant role in detector channel occupancy (the number of "hits" registered). It is also related to the particle fluence through detector elements and total ionizing dose received therein.

\subsubsection{The Inner Detector}

The Inner Detector (Figure 1.4) can be divided into three subsystems implementing two detector technologies. It is responsible for the precise measurement of particle tracks and momentum in order to reconstruct primary and secondary vertices. Closest to the beam line are the pixel detector and the Semi-Conductor Tracker (SCT). These are both built around solid state detector technologies with the minor difference between the two being that the pixels of the SCT are elongated into strips providing a rougher 2D coordinate. The pixel detector and the SCT occupy the region $33 \mathrm{~cm}$ to $51 \mathrm{~cm}$ away from the beam line and cover a pseudorapidity range of $|\eta| \leq 2.5$. Radially farther away from the beam line is the Transition Radiation Tracker 
(TRT) whose detecting element is essentially gas-filled straw tubes. The TRT takes up the remaining region designated for the ID from $55 \mathrm{~cm}$ to $108 \mathrm{~cm}$ and covers a pseudorapidity range of $|\eta| \leq 2.0$. [5]

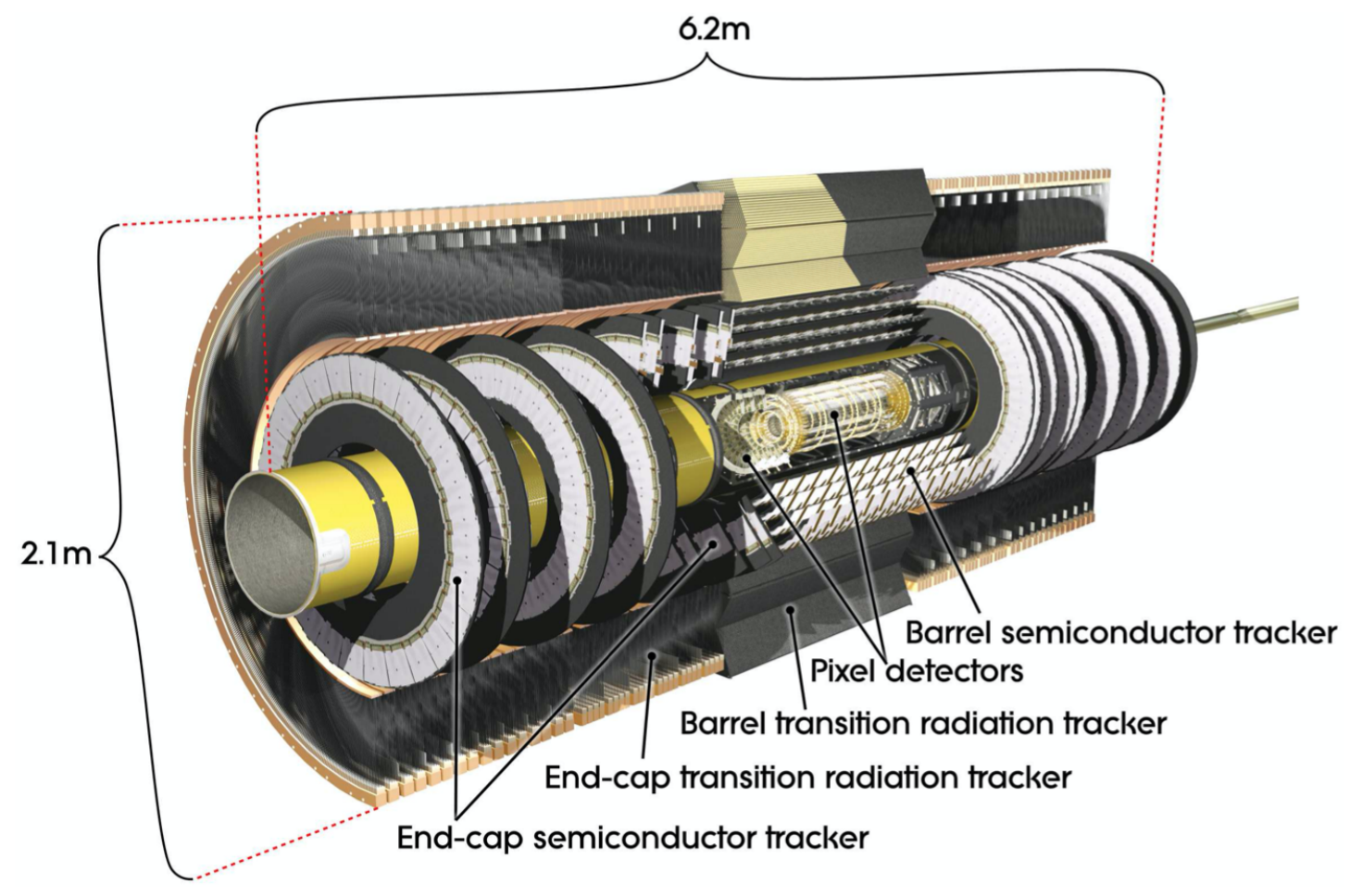

Figure 1.4: 3D cutaway of the ID. [3]

When compared to the target operational parameters of the HL-LHC the design limitations of the ID clearly reveal its deficiency. For example, the ID was designed to withstand a peak instantaneous luminosity of $1.0 \times 10^{34} \mathrm{~cm}^{-2} \mathrm{~s}^{-1}$ whereas the HL-LHC is expected to achieve $7.0 \times 10^{34} \mathrm{~cm}^{-2} \mathrm{~s}^{-1}$, a sevenfold disparity. The maximum pileup that can be handled by the ID is 40 , but the projected pileup during the HL-LHC operation is up to 200. Track and vertex finding efficiencies, as well as background rejection will all be fatally compromised by this increase in pileup.

Another area of high concern which necessitates the ID's replacement by the ITk is radiation hardness. The particle fluences expected over the operational period of the HL-LHC far outweigh the ID's design constraints. Deterioration in detector performance due to the harsh radiation environment of the HL-LHC would render the ID inoperable. The requirements for radiation hardness are outlined in section 1.4.3 and a more detailed description of the effects of ionizing radiation can be found in section 2.4. 


\subsection{The ITk Upgrade}

It is necessary for the ID's replacement to overcome the deficiencies outlined in the previous section. The performance requirements of the ITk are driven by the needs of the HL-LHC physics programme. In general, the ITk should have equal or better tracking performance compared to the ID despite an average pileup of 200. More specific performance requirements are further described in the discussion below. A more detailed description of the radiation environment expected within the ITk will be postponed until after the layout is explored in the following section (section 1.4.3).

\subsubsection{Performance requirements}

There are some general conditions which must be met by the ITk. It must be able to tolerate an instantaneous luminosity of up to $7.5 \cdot 10^{34} \mathrm{~cm}^{-2} \mathrm{~s}^{-1}$ with $25 \mathrm{~ns}$ bunch crossing for a total integrated luminosity of $3000 \mathrm{fb}^{-1}$. There must be full detector coverage (hermeticity) at each layer for all tracks with transverse momentum $\left(p_{T}\right)$ greater than $1 \mathrm{GeV}$ and originating from a $2 \mathrm{~mm}$ radius by $300 \mathrm{~mm}$ long cylinder around the centre of the ITk. Furthermore, there should be no abrupt reduction in tracking performance in the longitudinal direction. The layout must accommodate the currently used $2 \mathrm{~T}$ solenoid magnet. Tracks of primary vertices should be accurately reconstructed up to $\eta=4.0$, a significant improvement over the ID. Finally, the ITk should be robust in its tracking performance. Specifically, performance should not significantly deteriorate when $10 \%$ of the channels (randomly) die and under 10\% structural (correlated) failure of individual components.

Table 1.1 outlines the track reconstruction efficiencies for various particles. These efficiencies apply even under the maximum pileup of 200 and under the aforementioned maximum failure. Note that these efficiencies apply for $\eta<2.7$. A discussion of the large $\eta$ requirements will follow. The detector track reconstruction efficiencies should be uniform in $\phi$. Two track separation resolution is also required in order to measure tracks in cores of high energy jets. Lastly, average track reconstruction efficiency for primary hadrons should not decrease by more than $1 \%$ from low $p_{T}$ up to $p_{T}=1 \mathrm{TeV}$.

\begin{tabular}{lccc}
\multirow{2}{*}{ Particle } & \multirow{2}{*}{ Range } & \multicolumn{2}{c}{ Required Efficiency } \\
& & $|\eta| \leq 1.0$ & $1.0<|\eta|<2.7$ \\
\hline Muon & $>3 \mathrm{GeV}$ & $>99 \%$ & $>99 \%$ \\
Pion & $>1 \mathrm{GeV}$ & $>90 \%$ & $>85 \%$ \\
Electron & $>5 \mathrm{GeV}$ & $>90 \%$ & $>85 \%$ \\
\hline
\end{tabular}

Table 1.1: Target track finding efficiencies. [6]

One particular advantage the ITk will have over the ID is a significant improvement to coverage of large $\eta$. This coverage is useful to a large range of physics analyses, especially vector boson fusion (VBF). The extended range also significantly benefits forward jet reconstruction, missing transverse energy $\left(E_{T}^{m i s s}\right)$ resolution, and pileup jet rejection [6]. In this forward region and in the case of a typical physics process 
benchmark, the requirement for track reconstruction efficiency is $>80 \%$ for $t \bar{t}$ events at full pileup. When compared to tracking at $\eta<2$, the extended $\eta$ coverage increases acceptance for VBF channel $H \rightarrow$ $W W \rightarrow l \nu l \nu$ by a factor of 2 [7].

\subsection{ITk Physical Layout}

The ITk can be divided into two sub-detector systems, the strip and pixel detectors which are both comprised of barrel and endcap regions. The innermost sub-detector employs silicon pixel detectors while the outermost sub-detector relies on silicon strip detectors, hence the naming convention. The focus of this manuscript is the strip detector and its sensors, however, the basic principle of operation for both types of sensor (strip and pixel) is essentially the same (see section 2.3)

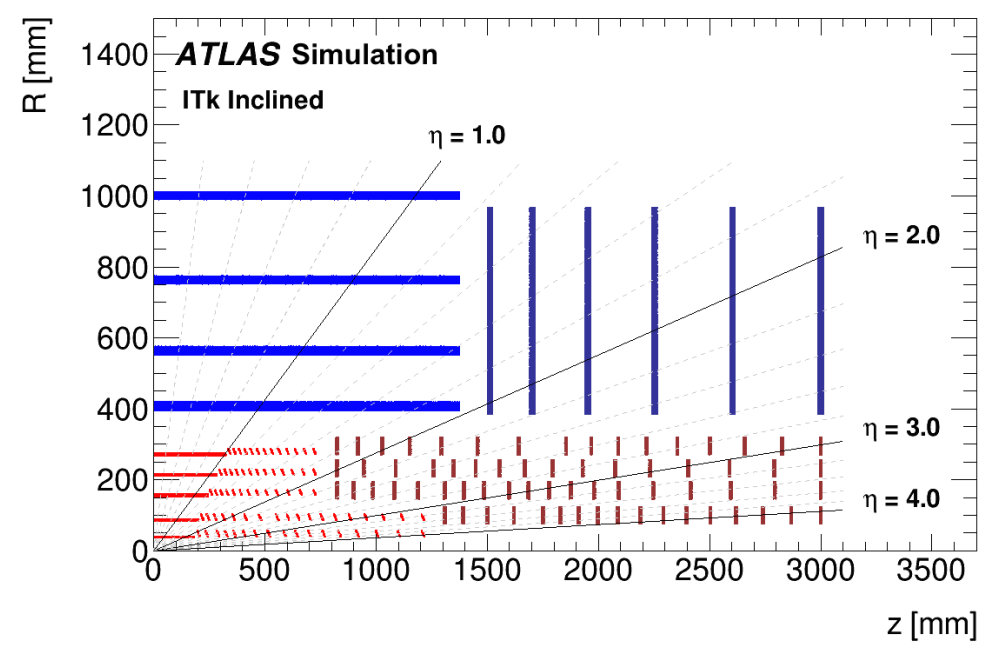

Figure 1.5: $2 \mathrm{D}$ schematic layout of ITk. The layout is mirrored across the $R$-axis. The strip and pixel subdetector systems are shown in blue and red, respectively. In both cases, the darker shaded colour identifies the endcap region. [6]

The aforementioned barrel region is the central region of the ITk and is made up of cylinders concentric to the beam line at various radii. Circular disks sitting in the transverse plane and perpendicular to the beam line make up the end-cap region. Figure 1.5 shows a quadrant of a schematic layout of the ITk with the strip and pixel detectors shown in blue and red, respectively. The layout is mirrored across the R-axis and rotated around the z-axis (see also Figure 1.6). The transition from barrel to end-cap is more complex in the pixel detector (see discussion below). 


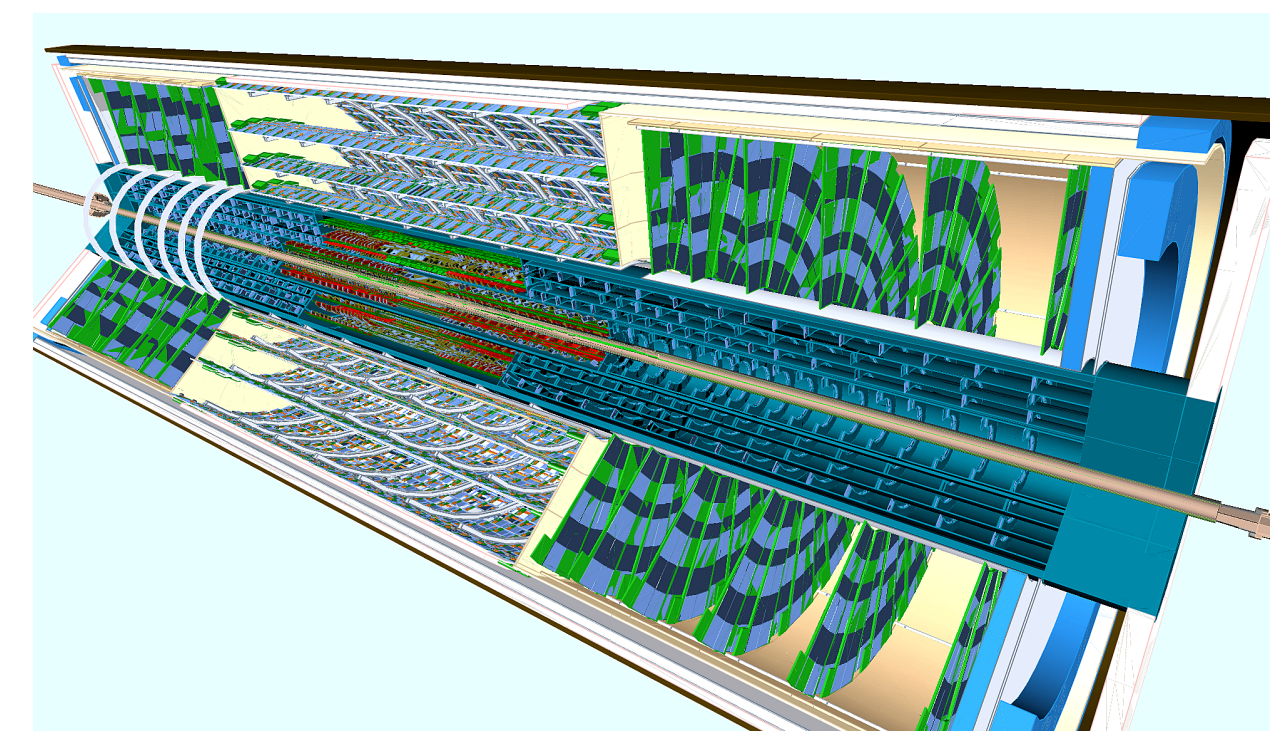

Figure 1.6: 3-D visualization of the ITk detector. The pixel detector is shown in red and dark blue (barrel and endcap) while the strip detector elements are given a light blue green colour scheme. [6]

\subsubsection{Strip Detector Layout}

Being set further away from the beam line, in the radial region extending from $405 \mathrm{~mm}$ to $1000 \mathrm{~mm}$, allows the strip detector to have the simpler of the two layouts while still meeting the previously discussed performance requirements. It has four barrel layers, each extending from $-1400 \mathrm{~mm}$ to $+1400 \mathrm{~mm}$ in the $z$-direction (along the beam line). The two innermost barrel layers, layers 0 and 1, are equipped with short strip sensors while the two outermost layers, layers 2 and 3, are equipped with long strip sensors. The barrel layers are made up of long, rectangular "staves" of single-file square strip sensors. These staves have sensors on either side rotated with respect to each other by $52 \mathrm{mrad}$ ( $26 \mathrm{mrad}$ on each side of a stave). This "stereo" angle allows for the second coordinate measurement. Finally, to achieve hermetic coverage for tracks down to $1 \mathrm{GeV}$, the staves are titled in the $R-\phi$ plane which allows the overlapping of neighbouring staves. The particular layout parameters for the barrel section are tabulated below (Table 1.2).

\begin{tabular}{cccccc} 
Layer & Radius $[\mathbf{m m}]$ & Channels in $\phi$ & Strip Pitch $[\mu \mathbf{m}]$ & Strip Length & Tilt Angle $\left[{ }^{\circ}\right]$ \\
\hline 0 & 405 & $28 \times 1280$ & 75.5 & 24.1 & 11.5 \\
1 & 562 & $40 \times 1280$ & 75.5 & 24.1 & 11.0 \\
2 & 762 & $56 \times 1280$ & 75.5 & 48.2 & 10.0 \\
3 & 1000 & $72 \times 1280$ & 75.5 & 48.2 & 10.0 \\
\hline
\end{tabular}

Table 1.2: Layout parameters for barrel section of strip detector. [6]

Six identical end-cap disks are arranged on either side of the barrel section between $1500 \mathrm{~mm}$ and 3000mm. Each end-cap is made up of wedge-shaped "petals". For the end-cap system, 6 novel sensor geometries have 
been developed (section 3.1) which maximize sensor coverage on each petal. By design, the wedge-shaped geometry also lends a stereo angle of $40 \mathrm{mrad}$ without the need to physically rotate the sensors. Optimizing strip lengths to keep strip occupancy below $1 \%$ was also a primary consideration for the sensor design. The strip lengths vary with a general trend of increasing the further the sensor is from the beam line. More particular layout parameters for the end-cap section are given below (Table 1.3).

\begin{tabular}{ccccc} 
Ring & Row & Inner Radius [mm] & Strip Length [mm] & Strip Pitch $[\mu \mathbf{m}]$ \\
\hline \hline Ring 0 & Row 0 & 384.5 & 19 & 75.0 \\
& Row 1 & 403.5 & 24 & 79.2 \\
& Row 2 & 427.5 & 29 & 74.9 \\
& Row 3 & 456.4 & 32 & 80.2 \\
\hline Ring 1 & Row 0 & 489.8 & 18.1 & 69.9 \\
& Row 1 & 507.9 & 27.1 & 72.9 \\
& Row 2 & 535.0 & 24.1 & 75.6 \\
& Row 3 & 559.1 & 15.1 & 78.6 \\
\hline Ring 2 & Row 0 & 575.6 & 30.8 & 75.7 \\
& Row 1 & 606.4 & 30.8 & 79.8 \\
\hline Ring 3 & Row 0 & 638.6 & 32.2 & 71.1 \\
& Row 1 & 670.8 & 26.2 & 74.3 \\
& Row 2 & 697.1 & 26.2 & 77.5 \\
& Row 3 & 723.3 & 32.2 & 80.7 \\
\hline Ring 4 & Row 0 & 756.9 & 54.6 & 75.0 \\
& Row 1 & 811.5 & 54.6 & 80.3 \\
\hline Ring 5 & Row 0 & 867.5 & 40.2 & 76.2 \\
& Row 1 & 907.6 & 60.2 & 80.5 \\
\hline
\end{tabular}

Table 1.3: Layout parameters for encap section of strip detector. [6]

\subsubsection{Pixel Detector Layout}

The Pixel detector sits nearest to the beam line occupying the full $6000 \mathrm{~mm}$ in the z-direction and out to $300 \mathrm{~mm}$ radially (see Figure 1.5) providing full hermetic coverage up to $|\eta|=4$. Unlike the Strip detector, the Pixel layout relies on a smoother "inclined" transition between the barrel and endcap sections in order to optimize tracking efficiency and channel occupancy, especially at large $\eta$. Inclined sensors in the forward region of the barrel section have a $56^{\circ}$ tilt in the $r-z$ plane. The non-inclined barrel sensor are tilted in $R-\phi$ by $14^{\circ}$ similar to the barrel sensors of the Strip detector. For technical reasons, namely access for maintenance or potential replacement, the two innermost barrel layers and innermost endcap ring are placed in an Inner Support Tube (IST). The remaining barrel layers and endcap rings, designed to last the entire lifetime of the HL-LHC, are grouped between the IST and the Pixel Support Tube (PST). The pixel size is $50 \times 50 \mu \mathrm{m}^{2}$ with sensor thickness $100 \mu \mathrm{m}$ in the IST and $150 \mu \mathrm{m}$ between the PST and IST. 


\subsubsection{Radiation Environment}

The expected levels of radiation in the ITk due to the increased luminosity of the HL-LHC are nearly an order of magnitude higher compared to the current ATLAS ID. Table 1.4 outlines the maximum particle fluences and total ionizing dose assuming an integrated luminosity of $3000 \mathrm{fb}^{-1}$, and a p-p inelastic crosssection of $79.3 \mathrm{mb}$ at centre-of-mass energy $\sqrt{s}=14 \mathrm{TeV}$. Fluences in the strip detector are dominated by particles from interactions in the calorimeter, beam-line, and ITk service material. For this reason and to minimize background radiation, ITk services are routed radially away from the beam line as soon as possible.

A safety factor of 1.5 has been applied to all radiation simulations. The 1.5 factor aligns with comparisons between fluence predictions and measurements made during Run-1 at $\sqrt{s}=7 \mathrm{TeV}$ and $8 \mathrm{TeV}$ which showed typical agreement better than $30 \%$ [8]. Furthermore, irradiation tests at up to twice the expected fluence have been performed to ensure there is no sudden degradation near the specified dose limit.

\begin{tabular}{lccc} 
Layer & Radius $[\mathrm{mm}]$ & Max. Fluence $\left[n_{e q} / \mathrm{cm}^{2}\right]$ & Max. Dose [MRad] \\
\hline \hline Strips & & & \\
\hline Long Strips & 762 & $3.8 \times 10^{14}$ & 9.8 \\
Short Strips & 405 & $7.2 \times 10^{14}$ & 32.5 \\
Endcap & 385 & $1.2 \times 10^{15}$ & 50.4 \\
\hline Pixels & & & \\
\hline Layer 0 & 39 & $1.87 \times 10^{16}$ & 549 \\
Layer 1 & 75 & $0.59 \times 10^{16}$ & 129 \\
Layer 2 & 155 & $0.22 \times 10^{16}$ & 87 \\
Layer 3 & 213 & $0.15 \times 10^{16}$ & 53 \\
Layer 4 & 271 & $0.11 \times 10^{16}$ & 477 \\
Endcap & 80 & $0.62 \times 10^{16}$ & \\
\hline
\end{tabular}

Table 1.4: Expected fluence and dose distributions in ITk. [9],[10],[11]

\subsection{Expected Performance of the ITk}

This section outlines expected operational performance aspects of the ITk with the strip detector as the main focus. Occupancy, tracking performance, and primary vertex reconstruction are the foremost subjects of this section. It should be noted that although the reconstruction software is not yet optimal for the ITk, the results of Monte-Carlo events (based on Geant4 detector descriptions) suggest that performance adequate for the HL-LHC can still be confidently achieved. 


\subsubsection{Occupancy}

Occupancy is defined as the fraction of channels per module which register a hit and therefore need to be read out. The goal for the ITk is to keep the average occupancy at $1 \%$ and well below $90 \%$ per event. Occupancy values have been calculated for the ITk assuming 200 minimum-bias events and assuming the same detector read out mode as used for Run 2 (01X mode). In this mode, a hit is only counted if no hit in the channel was registered in the previous bunch crossing and regardless of a hit in the subsequent bunch crossing. The occupancy study focusses on the strip detector of the ITk since it is the occupancy "bottleneck" in the ITk. This is largely due to the much higher channel density in the pixel detector which results in a nearly tenfold smaller occupancy compared to the strip detector.
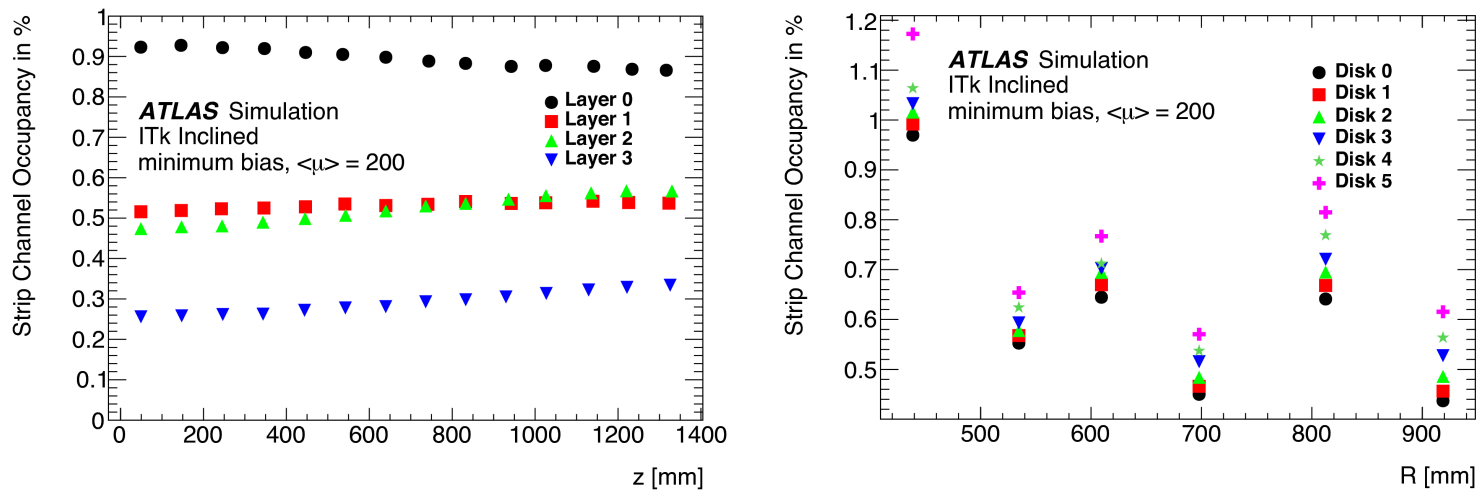

Figure 1.7: Channel occupancy from minimum bias events with $\langle\mu\rangle=200$ for the strip detector. Barrel (left) and endcap (right) regions are shown.[6]

Occupancy values are shown for the barrel and endcap in Figure 1.7 for $\langle\mu\rangle=200$. In the barrel, it is well below $1 \%$ in all layers and nearly independent of $\mathrm{z}$. Layer 0 obviously shows the highest occupancy values since it is nearest to the beam line. The hit density of layer 2 (further radially) is about a factor of two lower than layer 1 but the strip length is a factor of two larger. The result is that occupancy values in these two layers are nearly the same. In the endcap, occupancy is typically below $1 \%$ with the exception being at low radius, close to the beam line. There is generally higher occupancy in disk 5 caused by the backscattering of particles from the calorimeter endcap. Finally, the maximum channel occupancy per event is typically $4-10 \%$ (depending on the barrel layer or endcap ring) and well below the $90 \%$ constraint.

\subsubsection{Track Reconstruction}

The first step of track reconstruction is to group adjacent hits with collected charge above a set threshold into clusters (a description of how a "hit" is generated in the silicon pixel or strip detectors can be found in Chapter 2). A technique known as digital clustering is used in the ITk strip detector. Digital clustering determines a cluster position by taking the geometric mean of a collection of hits. It is clear that two (three) components of a cluster position are given simply by the strip (pixel) position. In order to achieve a three- 
dimensional readout in the strip detector, pairs of strip clusters on either side of a module are used. For example, in the barrel section strips run lengthwise roughly in the z-direction and the stereo angle between the pair of strip modules is used to more precisely measure the z-component of the cluster position.

Once their positions are determined, patterns of clusters (hits) can be used to construct track candidates. These candidates undergo further iterative processing to reduce incomplete and duplicate tracks, resolve situations where multiple tracks share common clusters, and remove fake tracks. Then, only after satisfying a set of quality and kinematic cuts can the candidates be considered as tracks. [12],[13]

\subsubsection{Tracking Efficiencies and Fake Rates}

Tracking efficiency and fake rate are two important considerations for a tracking detector. The definitions of each depend on the track reconstruction algorithms. This subsection will present how each are defined in the context of the ITk, operational expectations based on simulation, and comparison (in the case of efficiency) to Run 2 data.
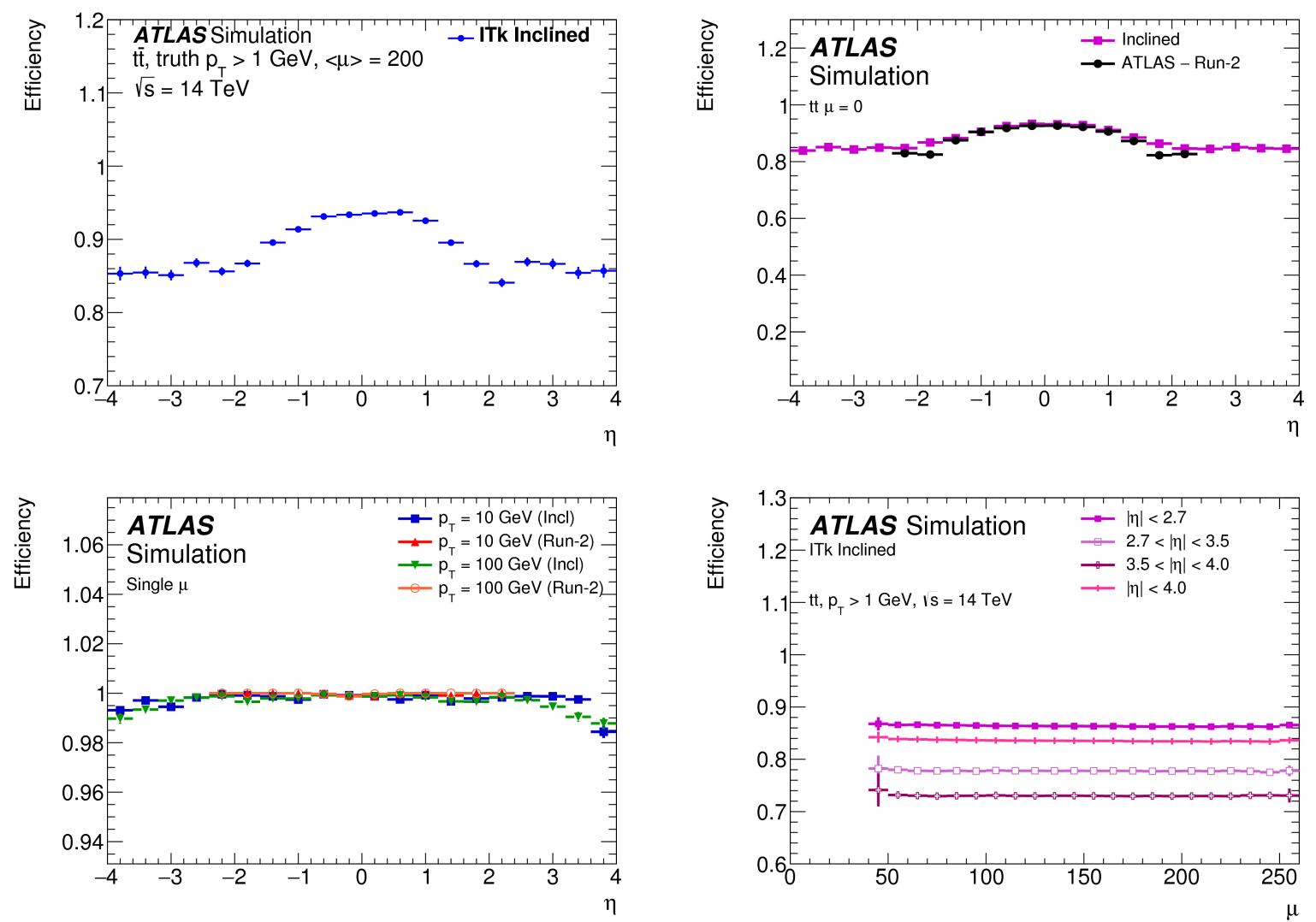

Figure 1.8: Track reconstruction efficiency as a function of $\eta$ for three scenarios (top left, top right, bottom left). The final plot (bottom right) indicates that efficiency is nearly independent of pileup in all $\eta$ regions.[6] 
Efficiency (equation (1.1)) is defined as the ratio between the number of selected reconstructed tracks which have been matched to a selected truth particle and the total number of selected truth particles.

$$
\epsilon_{\text {track }}=\frac{N_{\text {reco }}(\text { selected }, \text { matched })}{N_{\text {truth }}(\text { selected })}
$$

Selected truth particles are those which satisfy $\left|d_{o}^{\text {truth }}\right|<1.0 \mathrm{~mm},\left|z_{o}^{\text {truth }}\right|<150 \mathrm{~mm}, p_{T}>1 \mathrm{GeV}$, and $|\eta|<4$.0. They mush also be produced by primary interactions. Selected truth particles must also have a probability $>0.5$ of being matched to a track (this is called matching probability, $P_{\text {match }}$ ).

Figure 1.8 shows simulated efficiencies for various scenarios. The top left plot is efficiency as a function of $\eta$ for charged particles with $p_{T}>1 \mathrm{GeV}$ in $t \bar{t}$ events with $\langle\mu\rangle=200$. In the top right, the efficiency is shown for $\mu=0$ with a comparison to Run 2. Typical efficiencies of $99 \%$ for high $p_{T}$ muons is shown in the bottom left of the figure. Finally, the bottom right plot demonstrates efficiency being independent of pileup (in the range 40 to 260 ) for all $\eta$ ranges.

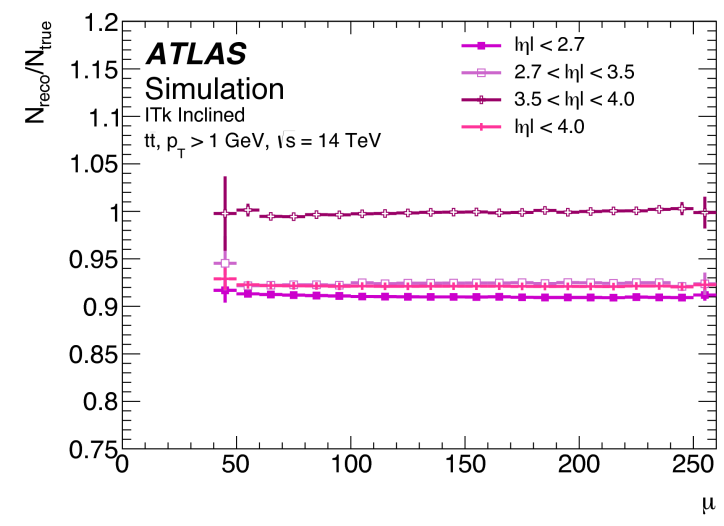

Figure 1.9: Ratio of reconstructed tracks to number of charged particles plotted against $\mu$. [6]

Fake tracks arise when clusters that belong to true charged particle tracks are randomly associated (noise hits also contribute to fake tracks, but to a far lesser extent). One way to define the fake rate is by considering the ratio of reconstructed tracks to the number of generated tracks (see Figure 1.9). The fake rate can also be assessed by considering the fraction of reconstructed tracks that do not match to truth particles. It should be noted that at higher pileup the potential for fake tracks increases as more clusters are produced increasing the vulnerability of the track reconstruction to find fake tracks. For the ITk this ratio is constant to within $1 \%$ in the range $40<\mu<260$.

\subsubsection{Track Parameter Resolution}

Five quantities are used by ATLAS to parameterize particle tracks. The first is particle charge divided by transverse momentum, $q / p_{T}$. Next, are the longitudinal and transverse impact parameters, $z_{o}$ and $d_{o}$ 

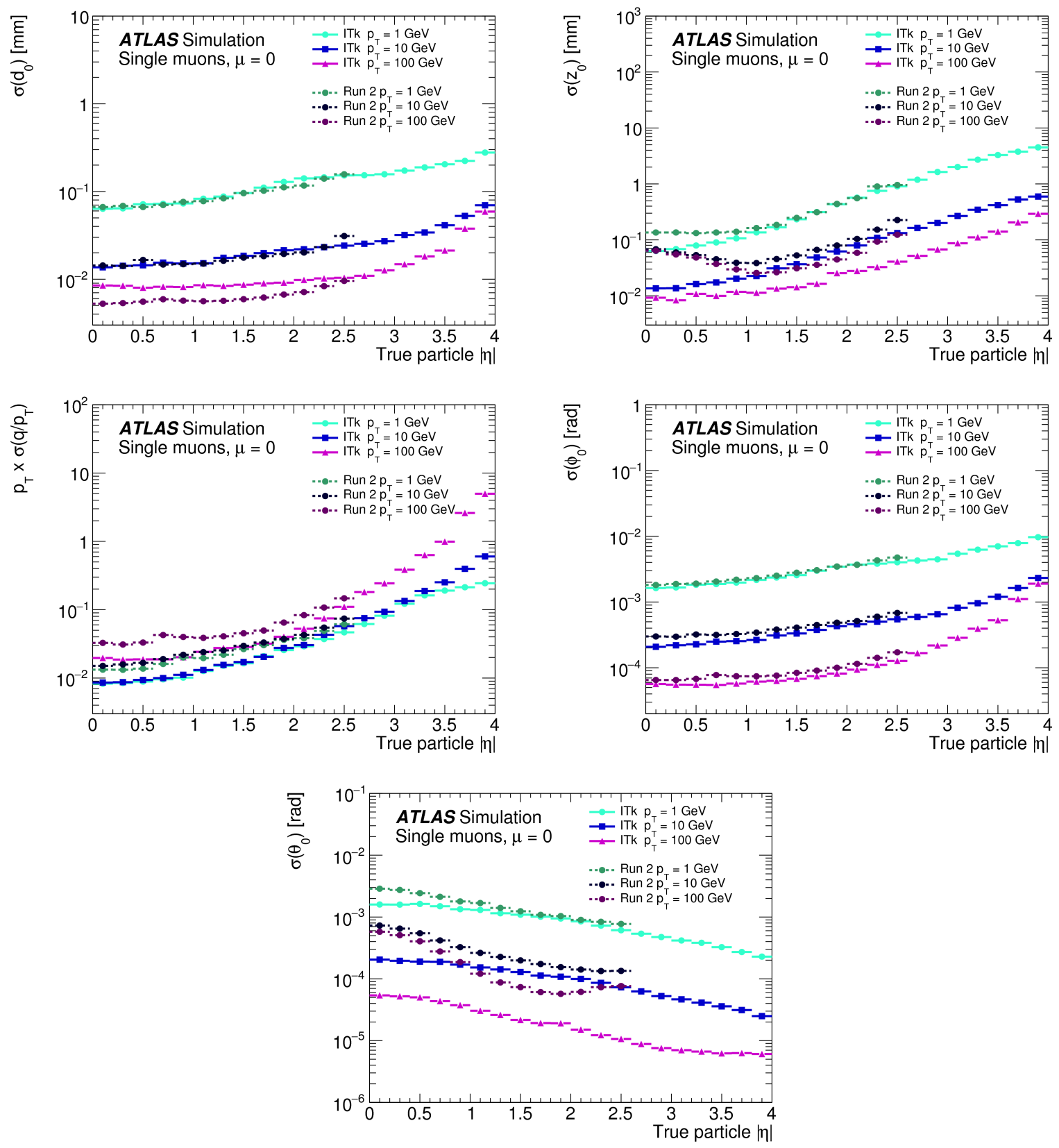

Figure 1.10: Resolutions shown for each of the five track parameters as a function of $\eta$. Comparisons to Run 2 data are also shown. [6] 
respectively, that provide an estimate of the track's origin. Lastly, are the polar and azimuthal angles, $\theta$ and $\phi$, which determine the track's direction. Through simulation and by applying the same selection criteria as for efficiency, the resolutions of these parameters are found by comparing reconstructed track parameters to their corresponding truth values.

The determination of the parameter resolutions is an iterative process which reduces any distortion of the RMS value arising from non-Gaussian tails. The process begins by calculating the RMS value of the parameter. Then, outliers beyond $3 \sigma$ are removed and the RMS value recalculated. This removal of outliers and recalculation of the RMS value is repeated until a stable RMS value is found.

Parameter resolutions for single muons against the true particle $\eta$ is shown in Figure 1.10 along with a comparison to results from Run 2 data. The resolutions of the ITk are typically better than that of Run 2 with the exception being the transverse impact parameter resolution at $p_{T}=100 \mathrm{GeV}$ where the resolutions are $20-30 \%$ worse. This is due largely to the use of digital clustering in the pixel detector ${ }^{1}$ and the larger inner radius of the first pixel layer. The longitudinal parameter resolution is notably better for the ITk in the central region and the polar angle resolution is also greatly improved, especially at higher $p_{T}$. These significant improvements are due largely to the smaller pixel pitch in the longitudinal direction which allows for charge sharing between pixels.

\subsubsection{Primary Vertex Reconstruction}

Once particle tracks are reconstructed they can be used to reconstruct primary vertices. This process, again, is iterative and consists of reducing the weight of outlying tracks and rejecting tracks which are incompatible with a vertex candidate. Rejected tracks are then used in the construction of new vertex candidates and the process is repeated until no additional vertex can be found or no tracks remain.

Figure 1.11 shows the simulated distribution of primary reconstructed vertices (left) and the number of reconstructed vertices versus $\mu$ for Run 2 (right). The average pileup in the sample is 200, implying that roughly $35 \%$ of the vertices are reconstructed. This is lower than the nearly $50 \%$ of reconstructed vertices during Run 2 (with average pileup 40). It can be seen in the right plot, however, that the number of reconstructed vertices does not increase linearly with pileup because as pileup increases more and more vertices are close together and begin to get merged. ${ }^{2}$

\footnotetext{
${ }^{1}$ During Run 2, the position determinations in the pixel detector were aided by a neural network which exploits the measured charge deposition. This yielded $10-20 \%$ better resolution than digital clustering alone. It is expected that this technique can also be applied to the ITk's pixel detector, however, the details of the pixel detector readout are not final, and so this study assumes a "worst case" scenario wherein the pixel detector only uses digital clustering.[6],[14]

${ }^{2}$ The importance of accurately determining the number of primary vertices is not entirely clear as the event-by-event pileup can be determined through other measures. For certain, the vertices of hard-scatter events must be determined with high efficiency and resolution since they are the input for various processes such as $b$-tagging, $t$ identification, and discrimination of prompt electrons and muons from those of $b$-hadron decays.[6]
} 

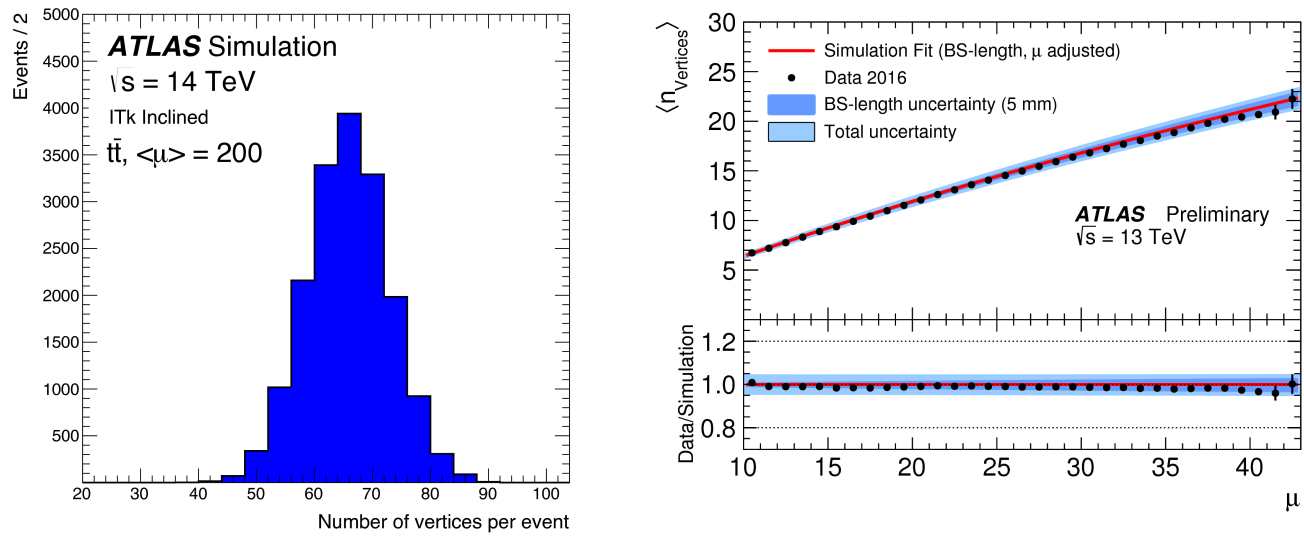

Figure 1.11: Distribution of primary reconstructed vertices per event in $t \bar{t}$ sample with $\mu=200$. [6] 


\section{Chapter 2}

\section{Semiconductor Theory}

At this point it is necessary to explore the the physics of semiconductor devices. The goal is not to provide an exhaustive description of semiconductor physics, but rather, to provide the background needed understand the fundamental and underlying principles behind sensor operation for the ITk. This chapter begins with a brief overview of the band theory of solids followed by a description of semiconductor types. These sections set the stage to explore the $\mathrm{p}-\mathrm{n}$ junction and its application in sensor operation.

\subsection{Band Theory of Solids}

Band theory arises when studying solids, particularly those with a periodic crystal structure. The underlying assumption is that the effective potential from the perspective of the material's valence electrons can be well approximated by an infinite chain of potential wells. A quantum mechanical study of this configuration results in electron energy levels which are grouped into energy bands. Each band is separated by energy ranges which the electrons cannot possess called forbidden gaps. A given solid's conductivity then depends on the existence and size of these forbidden gaps.

In metals, either the valence band is not fully occupied or the allowed energy bands overlap such that there are no forbidden gaps. In this configuration, the material's valence electrons are essentially free constituting a "sea of electrons". Shown in Figure 2.1 (A and B), the topmost electron can easily be moved by very small amounts of energy to higher energy states. To put it another way, valence electrons can gain enough kinematic energy to move throughout the crystal lattice structure. Once the topmost electron is promoted, the subsequent electron has an available energy level into which it can move and so on.

In insulators, the forbidden gaps are very large. This is caused by very strong bonds between valence electrons of neighbouring atoms in the lattice. As depicted in Figure 2.1 (C), all levels in the valence band are full while all levels in the conduction band are vacant. With a large energy gap, it is nearly impossible to promote even the topmost electron to the next available energy state. The result is that no valence electron can gain enough kinematic energy to move throughout the lattice, hence, conduction is impossible.

An intermediary case, designated as semiconductors, is one where the energy bands do not overlap but 


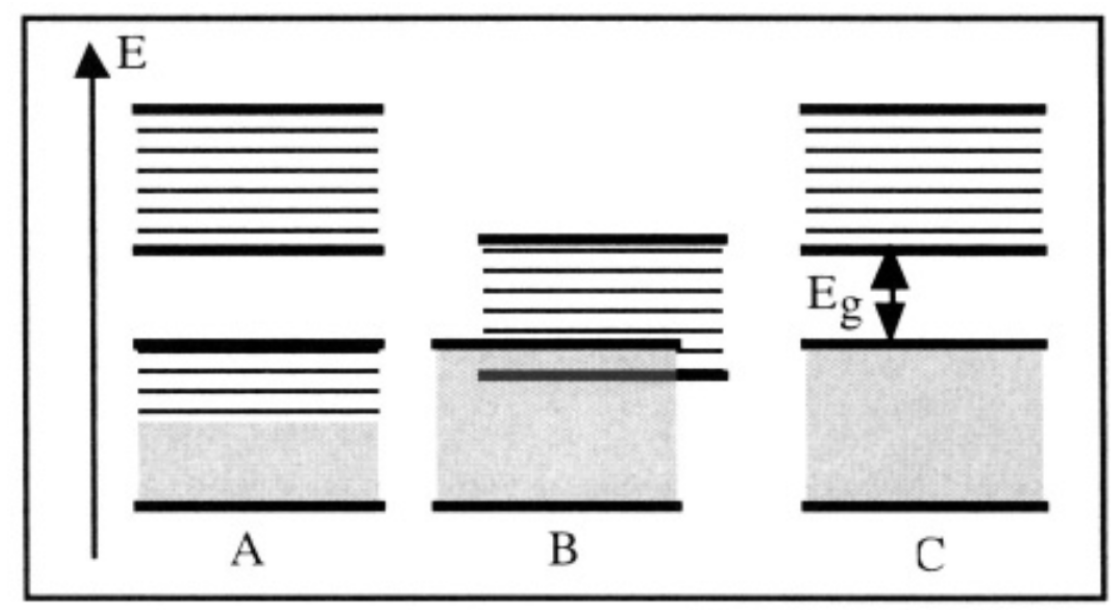

Figure 2.1: Schematic representation of energy band structure of solids. A and B demonstrate two configurations leading to conducting materials. $\mathrm{C}$ demonstrates a configuration leading to insulators or semiconductors depending on the size of the energy gap $\left(\mathrm{E}_{g}\right)$. [15]

the forbidden gap between the conduct band and the valence band is very small, on the order of thermal energies. This allows some (usually very few) electrons to be thermally excited into the conduction band. In this instance the thermally excited electrons, now promoted to the conduction band, can move freely through the material. There are also holes in the valence band left behind by the excited electrons. Additional conduction takes place when these holes are filled by other valence electrons "jumping" from a neighbouring bond in the crystal lattice to the position of the hole. [15],[16]

\subsection{N-type and P-type Semiconductors}

The picture is more or less complete for the two cases of insulators and conductors, but there are two more sub-cases to be explored in semiconductors. These cases arise when a new material is introduced with regularity into the semiconductor. This is known as doping, and the newly added material is referred to as the dopant.

A dopant material may be chosen such that its valence energy level is in the forbidden region of the bulk material just below the conduction band. Electrons in the valence level of the dopant can be thermally excited into the conduction band of the bulk material lending to the conductivity of the material. In this scenario the dopant is said to have donor energy levels and the material is referred to as an $n$-type ( $n^{+}$-type for heavy doping) semiconductor (Figure 2.2 top and middle).

Alternatively, a dopant material may have a vacant energy level within the forbidden region of the bulk material just above the valence band. Here, the electrons in the valence band of the bulk can be excited into the available energy level of the dopant leaving behind conducting holes in the valence band. The dopant is now said to have acceptor energy levels and the material is referred to as a $p$-type $\left(p^{+}\right.$-type, again for heavy 
doping) semiconductor.

Figure 2.2 shows one example each of an n-type and p-type semiconductor. The n-type semiconductor is silicon which has been doped with arsenic. The valence electron of arsenic, being not strongly bound, can very easily be excited into the conduction band of the silicon bulk. This leaves behind a positive arsenic ion and allows conduction to take place. In the second example, the dopant is boron which has a vacant energy level just above the valence band of the silicon bulk. Excitation of electrons into this vacant level produce negative boron ions leaving behind conducting holes in the valence band of the bulk.
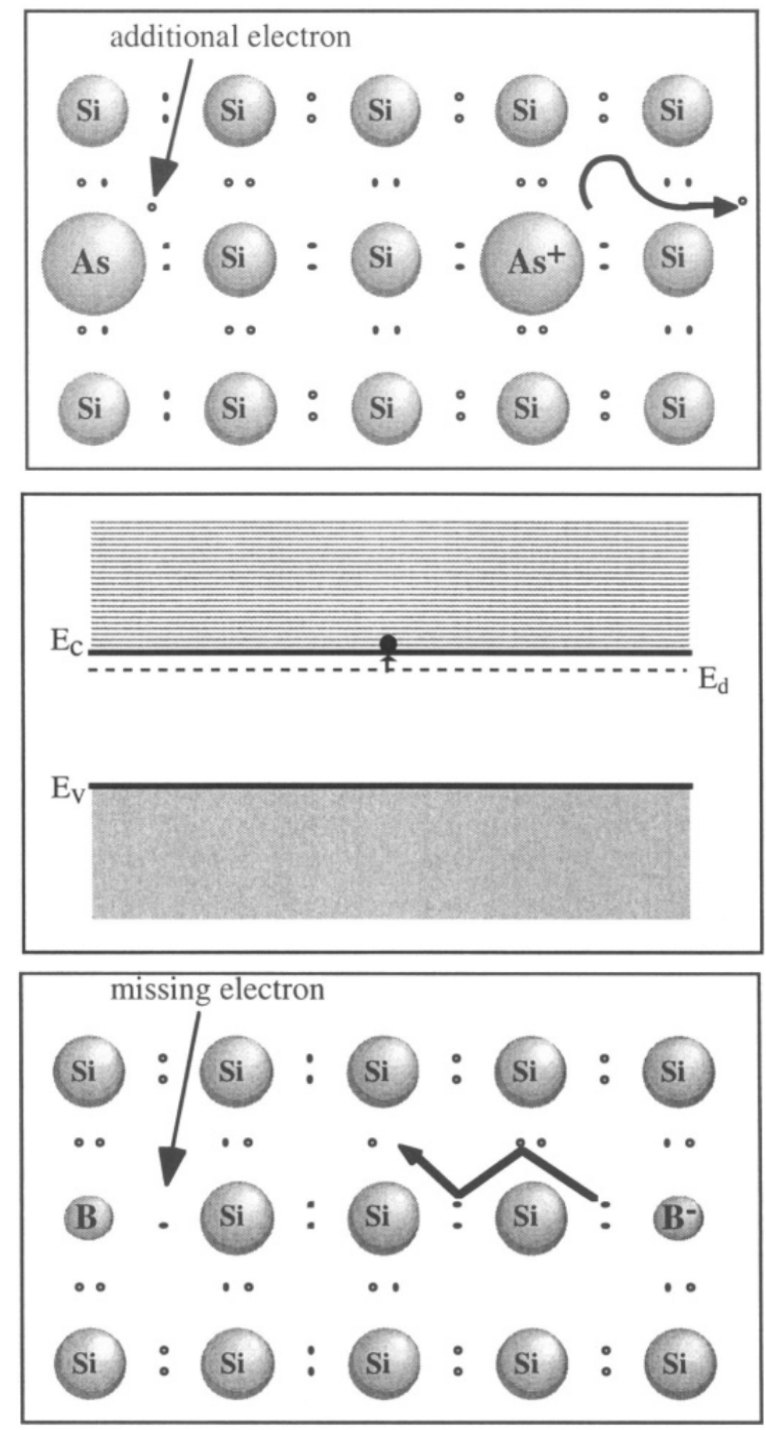

Figure 2.2: Examples of $\mathrm{n}$ and p-type semiconductors (top and bottom, respectively). The band structure for the n-type material is also shown with the donor level just below the conduction band (middle). [15] 


\subsection{The P-N Junction and Biasing}

The p-n junction is the fundamental technology at work in the ITk's micro-strip (and pixel) sensors. Following the brief discussion of semiconductor types it is now necessary to gain, at very least, a basic understanding of the p-n junction.

\subsubsection{General Characteristics}

As its name suggests, the p-n junction consists of two semiconductors of opposite types meeting at an interface. As electrons from the n-type material and holes from the p-type material attempt to diffuse across the interface, they will recombine with one another. Immobile donor and acceptor ions will then be left behind setting up a space charge. The space charge, being positive on one side of the interface and negative on the other side, will result in a net electric field which opposes the motion of the remaining holes and electrons (due to a Coulombic attraction to the ions). The electric field will continue to grow until equilibrium when it can completely oppose the attraction between electron-hole pairs preventing any more recombination.

The region devoid of mobile charge on either side of the interface is called the depletion region (sometimes called the depletion zone or space charge region). The size of the depletion region depends on physical properties such as doping concentration and charge mobility of the two materials selected. The size of the depletion region can, however, be increased or decreased through biasing, that is the application of an external electric potential across the interface. In a forward biased state the applied potential opposes the potential already set up in the depletion region. This means that the depletion region will decrease in size and eventually vanish at large enough forward bias. Conversely, a reverse biased sate is one where the applied potential reinforces the built-in potential causing the depletion region to grow.

The principal characteristic of the p-n junction is that it allows significant conduction in one direction

only. The condition for conduction is a forward bias which is larger than the built in potential of the junction. Under such a bias the depletion region vanishes and charge carriers are allowed to freely traverse the material. It is possible, however, for a junction to conduct in the reverse direction given large enough reverse bias. This phenomenon of "backward" conduction is known as junction breakdown.

The dominant mechanism of breakdown is called avalanche breakdown. In this mechanism, electronhole pairs are produced by thermal means. Under some voltage threshold, the production of electron-hole pairs simply leads to the normal reverse or leakage current. Above this threshold, the charge carrier (an electron and a hole) will be accelerated by the electric field and gain sufficient kinetic energy to break $\mathrm{Si}-\mathrm{Si}$ bonds. This leads to the formation of other electron-hole pairs which can, in turn, repeat this process.

A second mechanism which contributes to junction breakdown is Zener breakdown. Under high enough reverse bias voltages the covalent Si-Si bonds can be distorted to the point of being "torn" apart. Again, these broken bonds lead to conduction of electrons and holes. In the band representation, Zener breakdown corresponds to an electron tunneling through the band gap. Zener breakdown typically becomes more 


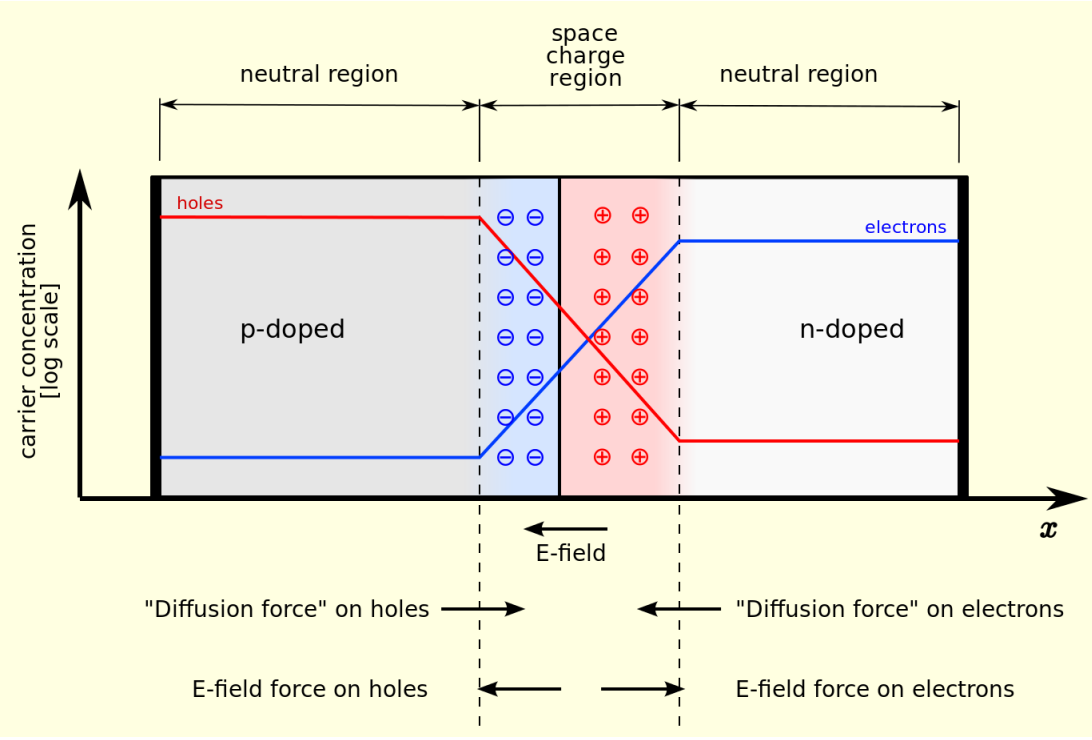

Figure 2.3: A p-n junction where electron and hole concentrations are shown schematically. Left behind dopant ions are also depicted in the depletion region. [17]

significant in materials with high doping concentrations.

\subsubsection{The Step Junction and Depletion Width}

The configuration of bringing two semiconductor materials, one n-type and one p-type, into contact with one another as previously described is known as a step junction. Although it is not necessarily practical to bring two individual materials into contact with one another, this scheme provides a useful illustration to understand the principles involved. Furthermore, step junction properties adequately resemble bulk properties of silicon strip sensors and so it is a beneficial exercise in this context.

Consider Poisson's equation reduced to one dimension since, for this setup, it is assumed that the potential varies only in one direction:

$$
\frac{d^{2} \phi}{d x^{2}}=-\frac{\rho}{\epsilon}
$$

Because the electric field is the gradient of the potential, this reduces to:

$$
\frac{d E}{d x}=\frac{\rho}{\epsilon}
$$

Where $E$ is the electric field, $\rho$ is the charge density, and $\epsilon$ is the permittivity of the material. The total width, $W$, of the depletion region will be equal to the sum of widths of the space charge regions on either side of the junction. These widths can be designated as $x_{n}$ for the width on the n-type side and $x_{p}$ for the width on the p-type side. Outside of the depleted region, there is no net charge so the electric field does not 
change. The charge density in the depletion region will be equal to the doping concentrations on each side of the junction (see Figure 2.4). The doping concentrations are denoted as $N_{D}$ for donor ions and $N_{A}$ for acceptor ions. For a constant doping concentration, it is easy to solve for the electric field on either side of the junction. First, the electric field will increase linearly up to the junction and then decrease linearly.
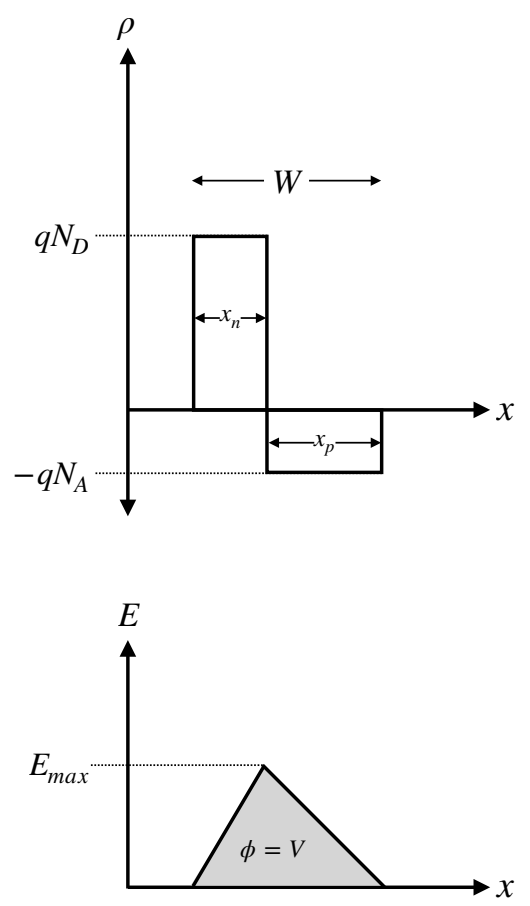

Figure 2.4: Schematic charge density and electric field strength vs distance in a p-n step junction.

So

$$
\begin{gathered}
d E=\frac{q N_{D}}{\epsilon} d x \\
E(x)=\frac{q N_{D}}{\epsilon} x
\end{gathered}
$$

in the space charge region on the n-type side of the junction and

$$
\begin{gathered}
d E=-\frac{q N_{A}}{\epsilon} d x \\
E(x)=-\frac{q N_{A}}{\epsilon} x
\end{gathered}
$$

in the space charge region on the p-type side of the junction. Imposing the requirement that the electric field 
be continuous at the boundary, it becomes apparent that the maximum electric field can be expressed as

$$
E_{\max }=\frac{q N_{A}}{\epsilon} x_{n}=\frac{q N_{D}}{\epsilon} x_{p}
$$

The total potential variation across the depletion region will be given by the integral of the electric field which is the area of the triangle with base $W$ and height $E_{\max }$

$$
V=\frac{1}{2} E_{\max } W
$$

Note that this potential is the total sum of potentials across the junction. Therefore, it will include the built in potential of the junction, $\phi_{b}$ (corresponding to the electric field of the space charge region at equilibrium), plus any voltage applied across the junction, $V_{R}\left(V_{R}\right.$ is chosen to denote reverse bias which will be additive to $\left.\phi_{b}\right)$. So, $V=\phi_{b}+V_{R}$.

Using the first definition of $E_{\max }$ from equation 2.5

$$
\begin{array}{r}
V=\frac{1}{2} \frac{q N_{A}}{\epsilon} x_{n}\left(x_{n}+x_{p}\right) \\
\\
\frac{2 V \epsilon}{q N_{A}}=x_{n}^{2}+x_{n} x_{p}
\end{array}
$$

and using the second definition

$$
\begin{array}{r}
V=\frac{1}{2} \frac{q N_{D}}{\epsilon} x_{p}\left(x_{n}+x_{p}\right) \\
\\
\frac{2 V \epsilon}{q N_{D}}=x_{p}^{2}+x_{n} x_{p}
\end{array}
$$

Adding equations 2.7 and 2.8 yields

$$
\begin{array}{r}
x_{n}^{2}+2 x_{n} x_{p}+x_{p}^{2}=\frac{2 V \epsilon}{q N_{D}}+\frac{2 V \epsilon}{q N_{A}} \\
W^{2}=\frac{2 V \epsilon}{q} \frac{N_{D}+N_{A}}{N_{D} N_{A}} \\
W=\sqrt{\frac{2 V \epsilon}{q} \frac{N_{D}+N_{A}}{N_{D} N_{A}}}
\end{array}
$$

This reduces when the combination of doping concentrations is rewritten as

$$
N_{e f f}=\frac{N_{D} N_{A}}{N_{D}+N_{A}}
$$


and finally

$$
\begin{array}{r}
W=\sqrt{\frac{2 V \epsilon}{q N_{e f f}}} \\
W=\sqrt{\frac{2\left(\phi_{b}+V_{R}\right) \epsilon}{q N_{e f f}}}
\end{array}
$$

So then it is clear that the depletion width for a step junction is proportional to the square root of the applied potential.

\subsubsection{Step Junction Capacitance Characteristics}

The final feature of the step junction which is instructive to study is the capacitance relationship. The capacitance per unit area is defined as

$$
\frac{C}{A}=\frac{d Q}{d V}
$$

where $C$ is the capacitance, $A$ the area, and $Q$ the charge per unit area. For simplicity, take the case of the step junction where $N_{A}>>N_{D}$ so that $N_{\text {eff }} \approx N_{D}$. The charge per unit area is $Q=q N_{D} W$ so that

$$
d Q=q N_{D} d W
$$

Next, equation 2.11 is rewritten so that $V$ is a function of $W$ and taking the derivative yields

$$
d V=\frac{q N_{D} W}{\epsilon} d W
$$

Putting these two together into equation 2.12 finally gives the capacitance per unit area

$$
\frac{C}{A}=\frac{\epsilon}{W}
$$

Interestingly, this is precisely the capacitance given by a parallel plate capacitor separated by distance $W$. By substituting in the equation for depletion width as a function of voltage, the capacitance voltage relationship of a step junction is obtained

$$
\frac{C}{A}=\sqrt{\frac{q \epsilon N_{e f f}}{2 V}}
$$

This relationship can be rewritten as

$$
\frac{1}{C^{2}}=\frac{2 V}{q \epsilon N_{e f f} A^{2}}
$$

or, equivalently

$$
\frac{1}{C^{2}}=\frac{2\left(V_{R}+\phi_{b}\right)}{q \epsilon N_{e f f} A^{2}}
$$


so that the effective doping concentration can be extracted from the linear slope of a plot of $1 / C^{2}$ vs $V_{R}$. [16]

\subsection{Principle of Operation for Silicon Strip Sensors}

The strip and pixel sensors of the ITk harness the p-n junction as a means to generate signals from passing charged particles. In fact, the detector's tracking potential is directly related to the depletion region and the p-n junction's diode nature. In the ITk sensors, thousands of strip (pixel) sensor implants are made of $\mathrm{n}^{+}$-type silicon while the bulk is comprised of p-type silicon. This sensor configuration is referred to as $\mathrm{n}^{+}$-in-p and is chosen primarily for the larger signal-to-noise ratio which can be achieved (as compared to the p-in-n technology used in the current ATLAS SCT) even after irradiation.

The ITk strip sensor design includes an intentional layer of $\mathrm{SiO}_{2}$ across the sensor surface which has many important roles. During the fabrication process, a "blank" wafer of p-type silicon is allowed to oxidize thermally. Small windows are then opened in the oxide layer allowing the strip implants to be formed through the diffusion of donor impurities into the exposed silicon. The oxide layer also mitigates surface effects in the semiconductor material allowing for better control over the device characteristics ${ }^{1}$. Lastly, the oxide layer serves as a dielectric between the implant and the strip metal. The result is an AC-coupled configuration which protects the readout electronics against leakage current.

A variety of other structures have been implemented into the sensor design. Poly-silicon bias resistors bridge the strip implants to a common bias rail in order to maintain a potential across each strip and the bulk. A $p$-stop structure made of heavily doped p-type material surrounds each strip to reduce surface currents, increase strip-to-strip isolation, and ultimately maximize the detector's spatial resolution. Punch-throughprotection (PTP) gated diode structures allow for (nearly) unresisted discharge of potentially damaging large deposits of excess charge which may arise at a particular strip.

A highly doped p-type backplane, the bottom of which has metallization, is also implemented. Ideally, the bias voltage is large enough to cause the depletion region to extend throughout the entire width of the sensor at which point the sensor is fully depleted. This maximizes the sensor's sensitivity but also results in very high electric field strengths. The backplane serves as the negative electrode and helps to ensure that the high field in the sensor bulk will be as uniform and vertical (from implant to backplane) as possible. Additionally, the backplane is electrically connected to an edge metal structure surrounding the sensor surface. This structure controls edge field effects and reduces high field density regions which can lead to premature sensor breakdown.

Figure 2.5 illustrates the basic principle of operation of a strip sensor. Reverse bias is applied to the sensor increasing the size of the depletion region. The passage of a charged particle through a material results in ionization along its path according to the Bethe-Bloch formula. When this passage occurs throughout the depleted region of a p-n junction mobile charge carriers (electrons and holes) are liberated. These charge

\footnotetext{
${ }^{1}$ Note that in the first section of this chapter, band theory was introduced with the assumption that the crystal lattice is periodic and extends infinitely. The physical termination of the crystal lattice is what leads to these pesky surface effects.
} 


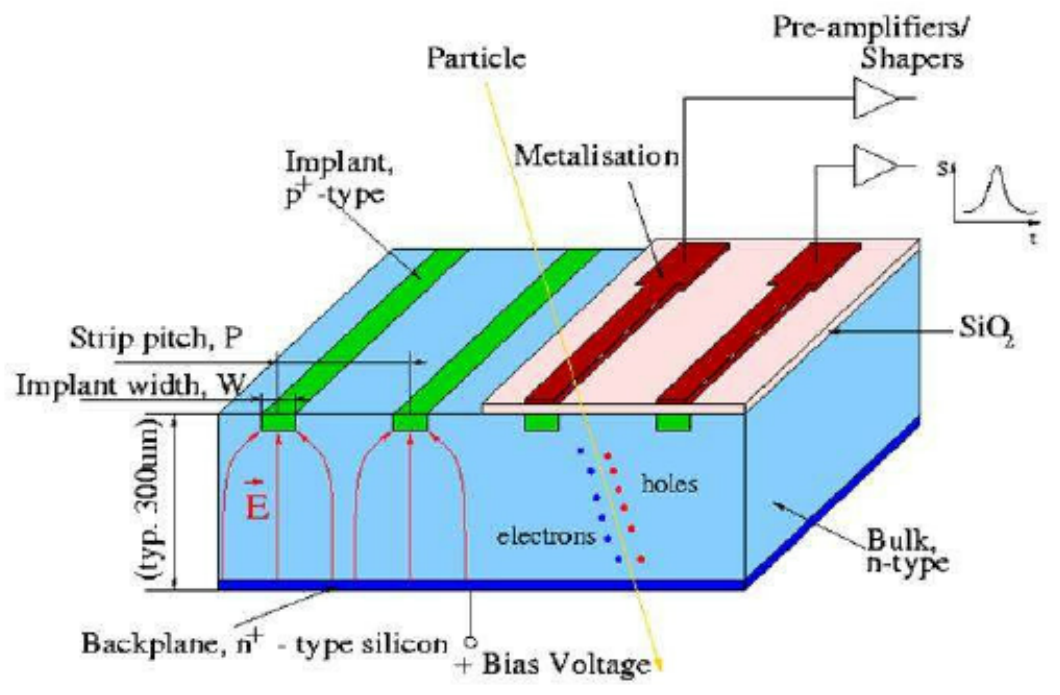

Figure 2.5: Schematic cross section of a $\mathrm{p}^{+}$-in-n silicon strip sensor. ITk sensors operate using the same basic principles but the types are reversed.[18]

carries will be swept away by the space charge field to electrodes maintaining the depletion region. The movement of charge to the electrodes induces a voltage pulse proportional to the magnitude of charge liberated by the passing charged particle. Finally, the induced voltage pulse can be interpreted by front-end readout electronics through discrimination against a set threshold in order to convert it to a digital "hit" for the associated channel.

\subsection{Effects of Radiation}

As mentioned earlier, radiation damage is a primary concern for the ITk. With a safety factor of 1.5 , the maximum expected fluence (dose) expected for the ITk strip detector as outlined in Table 1.4 is $1.2 \times$ $10^{15} n_{e q} / \mathrm{cm}^{2}$ (50.4MRad). Fluences of this magnitude translate to radiation damage which negatively impinges on the operation of the sensors. The damage incurred from radiation manifests either in the silicon bulk or in the surface oxide layer.

\subsubsection{Bulk Damage}

Bulk damage is the displacement of atoms in the crystal lattice and is the dominant effect which decreases the sensor performance over time. If enough energy is imparted to an atom during an interaction, it may subsequently remove additional atoms from their respective lattice sites before coming to rest. Bulk damage can thus be divided into two subcategories; point defects and cluster defects. A point defect occurs when just one atom is displaced from its lattice site while a cluster defect refers to a group of displaced atoms. 
The resultant vacancies and interstitial atoms form complex lattice defects whose behaviour is similar to impurities.

An interstitial atom may act similar to a donor or acceptor impurity. In this case, its energy levels can temporarily trap and subsequently release mobile charge carriers with some delay (on the order of $\mu \mathrm{s}$ ). This trapping action results in a change to the material's effective doping concentration. If the interstitial atom has an energy level near the middle of the forbidden gap it acts as a recombination or generation centre. These are sites where available energy levels in the band gap act as "stepping stones" which allow the transition of electrons or holes between the conduction and valence bands. The resultant effect here is a reduction of charge carrier lifetime and thus measured charge.

In silicon, radiation induced levels in the band gap are primarily acceptor states (p-type) [19]. This leads to type inversion which is the conversion from n-type silicon to effective $\mathrm{p}$-type after prolonged exposure to radiation. For this reason, the $\mathrm{n}^{+}$-in-p sensor configuration has an advantage in radiation hardness over alternative technologies such as $\mathrm{p}$-in-n or $\mathrm{n}^{+}$-in-n.

\subsubsection{Surface Damage}

While lattice displacements are a primary concern in the bulk, ionizing radiation is the dominant cause of degradation in the surface oxide layer, particularly when the dose is received with the sensor under bias. During irradiation, electron-hole pairs will be produced in the oxide. Under zero bias, these pairs quickly recombine and the material returns to its original, unaltered state. With some non-zero bias applied, the electric field in the oxide layer will pull the electron-hole pairs apart. The electrons will then be allowed to reach the positive electrode, the bias rail for the ITk sensors. The holes, however, may become trapped near the oxide-bulk interface. The ultimate result is a build up of positive space charge in the oxide surface which leads to deterioration in the sensor's performance.

Damage to the surface oxide layer through ionizing radiation has been shown to negatively influence total leakage current (which increases with increasing fluence) and inter-strip resistance (which decreases with increasing fluence) [20],[21],[22]. There is also a minor increase in inter-strip capacitance with increasing radiation fluences for voltages below full depletion. Lower leakage currents are desirable to minimize both the total current draw of the detector as well as the available charge carries which may contribute to avalanche breakdown. It is important to maintain high inter-strip resistances in order to maximize strip isolation and thus the detector's spatial resolution. Lastly, inter-strip capacitance contributes to the input capacitance of the readout electronics which is directly related to the detector noise. Therefore, it should be kept as low as possible. These sensor characteristics can all be used to identify potential changes in the surface oxide and are discussed in further detail in the following chapter. 


\section{Chapter 3}

\section{Sensor Testing}

With a general description of semiconductor physics, particularly as it relates to detector technology, the next pertinent topic is sensor testing. A suite of quality control (QC) tests have been established for the ITk strip sensors. The goal of these QC tests is to verify the manufacturer's specifications and ensure acceptable operational parameters. The chapter begins with a description of the sensor layouts used specifically in the ITk. This is followed by two sections, one each for physical properties and electrical tests, which outline in detail each of the QC tests associated therein.

\subsection{The ITk Sensor}

As previously discussed (see Chapter 2), the ITk sensors utilize an AC-coupled, $\mathrm{n}^{+}$-in-p configuration. There are eight sensor layouts used in total, two for the barrels and six for the end-caps, which are described in further detail below. Specifications common to all sensors, independent of the layout, are given in Table 3.1. Also common to all layouts is the implant and strip metal widths. An example cross section of the interstrip region is given in Figure 3.1. The strip metal includes $200 \mu \mathrm{m}$ long by $56 \mu \mathrm{m}$ wide $A C$ pads at either end of the strip. In addition, $60 \mu \mathrm{m}$ long by $50 \mu \mathrm{m}$ wide $D C$ pads at either end of the strip are also present. Having two sets of pads per strip allows one set to be used for direct sensor testing while the other set is left un-probed (and unscratched) providing a clean, smooth Al surface optimal for wire-bonding with the readout ASIC. Finally, there are visible chip boundary markers next to the $1^{\text {st }}$ and $128^{\text {th }}$ AC pads for each readout ASIC. The sensors implement bias resistors through poly-silicon implants connecting the strip implants to a common bias rail. Inter-strip isolation is enforced by $6-8 \mu \mathrm{m}$ wide p-stop implants which continuously surround each individual strip. A passivation layer is applied with uniform thickness over the strip side of the sensor with openings at every strip pad as well as on the bias rail and backplane top metal. ${ }^{1}$

\footnotetext{
${ }^{1}$ Some design features, such as passivation material/thickness and implant depth/doping, are proprietary to the manufacturer and have been intentionally omitted.
} 


\begin{tabular}{|c|c|}
\hline \multicolumn{2}{|l|}{$\begin{array}{l}\text { Substrate Material \& Mechanical } \\
\text { Specifications }\end{array}$} \\
\hline Wafer size \& Type & 6-inch/150mm \& p-type \\
\hline Physical thickness & $305-335 \mu \mathrm{m}$ \\
\hline Active thickness & $>90 \%$ physical thickness \\
\hline Thickness tolerance & $\pm 5 \%$ \\
\hline Sensor bow after process and dicing & $<200 \mu \mathrm{m}$ \\
\hline Resistivity & $>3.5 \mathrm{k} \Omega \mathrm{cm}$ \\
\hline Oxygen concentration & $1.5 \times 10^{16}$ to $6.5 \times 10^{17} \mathrm{~cm}^{-3}$ \\
\hline \multicolumn{2}{|l|}{ Specifications before irradiation } \\
\hline Maximum operating voltage & $500 \mathrm{~V}$ \\
\hline Full depletion voltage & $<350 \mathrm{~V}$ \\
\hline $\begin{array}{l}\text { Total initial leakage current } \\
\text { normalized to } 20^{\circ} \mathrm{C}\end{array}$ & $\begin{array}{l}<0.1 \mu \mathrm{A} / \mathrm{cm}^{2} \text { at } 500 \mathrm{~V} \text { tested with relative humidity }(\mathrm{RH}) \\
<20 \%\end{array}$ \\
\hline Leakage current stability & $\begin{array}{l}<15 \% \text { variance in temperature corrected leakage current } \\
\text { during a } 40 \text { hour period held at } 450 \mathrm{~V} \text { with } \mathrm{RH}<10 \%\end{array}$ \\
\hline Poly-silicon bias resistors & $1.5 \pm 0.5 \mathrm{M} \Omega$ \\
\hline Inter-strip resistance & $>10 \times R_{\text {bias }}$ at $300 \mathrm{~V}$ at room temperature $(\mathrm{RT})$ \\
\hline Inter-strip capacitance & $<1 \mathrm{pF} / \mathrm{cm}$ at $300 \mathrm{~V}$, measured at $100 \mathrm{kHz}$, at $\mathrm{RT}$ \\
\hline Coupling capacitance & $>20 \mathrm{pF} / \mathrm{cm}$ at $1 \mathrm{kHz}$ \\
\hline Resistance of Al read-out strip & $<30 \Omega / \mathrm{cm}$ \\
\hline Resistance of n-implant strip & $<50 k \Omega / \mathrm{cm}$ \\
\hline $\begin{array}{l}\text { Onset of micro-discharge } \\
\text { (breakdown) }\end{array}$ & $>500 \mathrm{~V}$ \\
\hline Number of strip defects & $\begin{array}{l}<1 \% \text { per segment and sensor } \& \text { no more than } 8 \text { consecutive } \\
\text { bad strips }\end{array}$ \\
\hline \multicolumn{2}{|l|}{$\begin{array}{l}\text { Specifications after irradiation } \\
\left(1.2 \times 10^{15} n_{e q} / \mathrm{cm}^{2}-50 \mathrm{MRad}\right)\end{array}$} \\
\hline Maximum operating voltage & $500 \mathrm{~V}$ \\
\hline $\begin{array}{l}\text { Total leakage current normalized to } \\
-20^{\circ} \mathrm{C}\end{array}$ & $<0.1 \mathrm{~mA} / \mathrm{cm}^{2}$ up to $500 \mathrm{~V}$ \\
\hline Leakage current stability & Same as pre-irradiation limit, normalized to $-20^{\circ} \mathrm{C}$ \\
\hline Inter-strip resistance & $>10 \times R_{\text {bias }}$ at $400 \mathrm{~V}$ at $-20^{\circ} \mathrm{C}$ \\
\hline Inter-strip capacitance & $<1 \mathrm{pF} / \mathrm{cm}$ at $400 \mathrm{~V}$, measured at $100 \mathrm{kHz}$, at $-20^{\circ} \mathrm{C}$ \\
\hline $\begin{array}{l}\text { Onset of micro-discharge } \\
\text { (breakdown) }\end{array}$ & lower of: $>500 \mathrm{~V}$ or $>50 \mathrm{~V}$ above full depletion voltage \\
\hline Inter-strip resistance & $>10 \times R_{\text {bias }}$ at $400 \mathrm{~V}$ and for $T=-20^{\circ} \mathrm{C}$ \\
\hline Collected charge & $>7500$ electrons per MIP at $500 \mathrm{~V}$ \\
\hline Number of strip defects & May increase, but must remain below the pre-irradiation limit \\
\hline
\end{tabular}

Table 3.1: Sensor specifications common to all sensor layouts. Unlisted irradiation specifications are the same as the pre-irradiation specifications. 


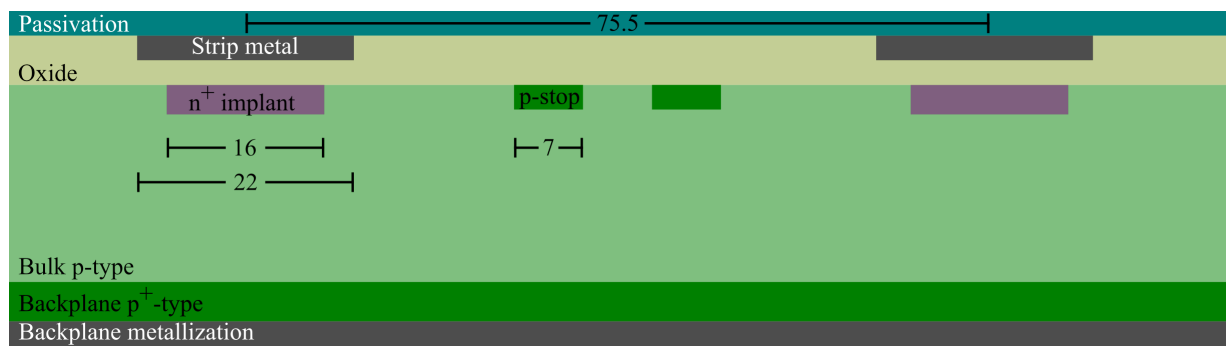

Figure 3.1: Example of a cross section through the interstrip region of a sensor. The strip pitch given is that of barrel sensors. The diagram is to scale in the horizontal direction only. All units are in micrometers.

\subsubsection{Barrel Sensor Layout}

The two barrel sensor layouts are designated as short strip (SS) and long strip (LS). Both layouts incorporate a nearly square sensor with dimensions $97.620 \mathrm{~mm} \times 97.950 \mathrm{~mm}$ (length $\times$ height). The distances from the inner edge of the bias rail to the cut sensor edge (after dicing) are $450 \mu \mathrm{m}$ (longitudinal) and $550 \mu \mathrm{m}$ (lateral). This so-called "slim edge" feature is intended to maximize the active area of the sensor. Sensors are divided into segments of 1280 parallel strips with a constant strip pitch of $75.5 \mu \mathrm{m}$. Two additional field-shaping strips (one per side) in each segment parallel with the other strips are also present. The SS layout hosts four segments with a strip length of $24.1 \mathrm{~mm}$ whereas the LS layout has only two segments but with a longer strip length of $48.2 \mathrm{~mm}$, hence the naming convention.

\subsubsection{End-cap Sensor Layout}

The six endcap sensor layouts are more complex than those of the barrel sensors and have been named R0-R5 (corresponding to the 6 endcap rings). A novel sensor geometry entitled stereo annulus has been implemented. The sensors are wedge shaped with circular arcs of a common centre (the beam pipe) forming the top and bottom edges. The strips are all directed to a point which is slightly displaced from the beam pipe centre. In this way the stereo angle of $40 \mathrm{mrad}$ between sensors on opposite sides of endcap petals is achieved.

The procedure for drawing the exact geometry of the endcap sensors is shown in Figure 3.2 (left). Points ABCD enclose the sensor area. The top and bottom circular arcs, CB and DA, share the common centre point, $\mathrm{O}$. The centre of the sensor, $\mathrm{O}_{\mathrm{w}}$, is given by the intersection of an arc of radius $\mathrm{R}$ with the vertical. Point $\mathrm{F}$ is then chosen so that the angle between the line segment $\mathrm{O}_{\mathrm{w}} \mathrm{F}$ and the vertical equals the stereo angle, $\phi_{s}$. Finally, all the strips and the straight sensor edges point toward F. While the arc or edge lengths may differ between layouts, every endcap sensor shares this common general geometry. Moreover, the stereo angle is also common to every layout.

Half of the layouts (R0, R1, and R3) have four segments of strips while the other half have only two. In either case, the strip length generally increases moving further from the beam line. Because of the wedge 
shape where strips are directed to a common point rather than being parallel to one another, the strip pitch varies across a sensor but is chosen to make it as close as possible to $75 \mu \mathrm{m}$. Additionally, the wedge shape causes variance in the number of strips per segment since, on a given sensor, segments further from the beam line have a larger area. It should be noted, however, that the strip length within a sensor segment is kept constant.
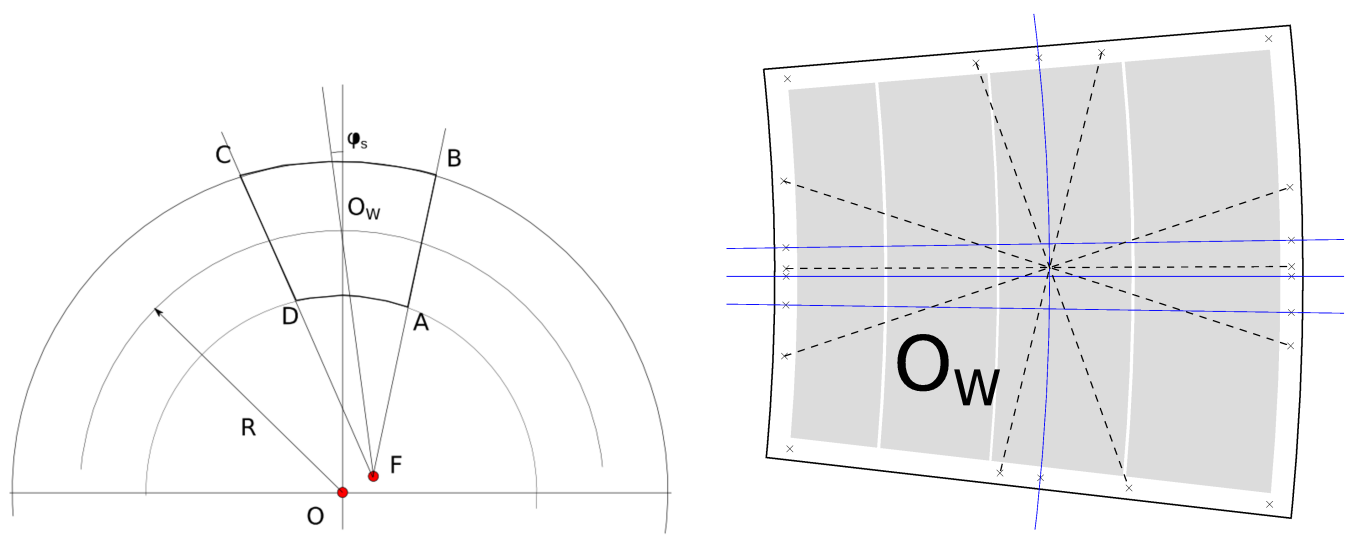

Figure 3.2: (Left) Demonstration of the stereo annulus geometry. (Right) The hashed lines define the centre of the sensor, $\mathrm{O}_{\mathrm{w}}$. The solid blue lines indicate the sensor's radial position in the global coordinates and on a petal. [6]
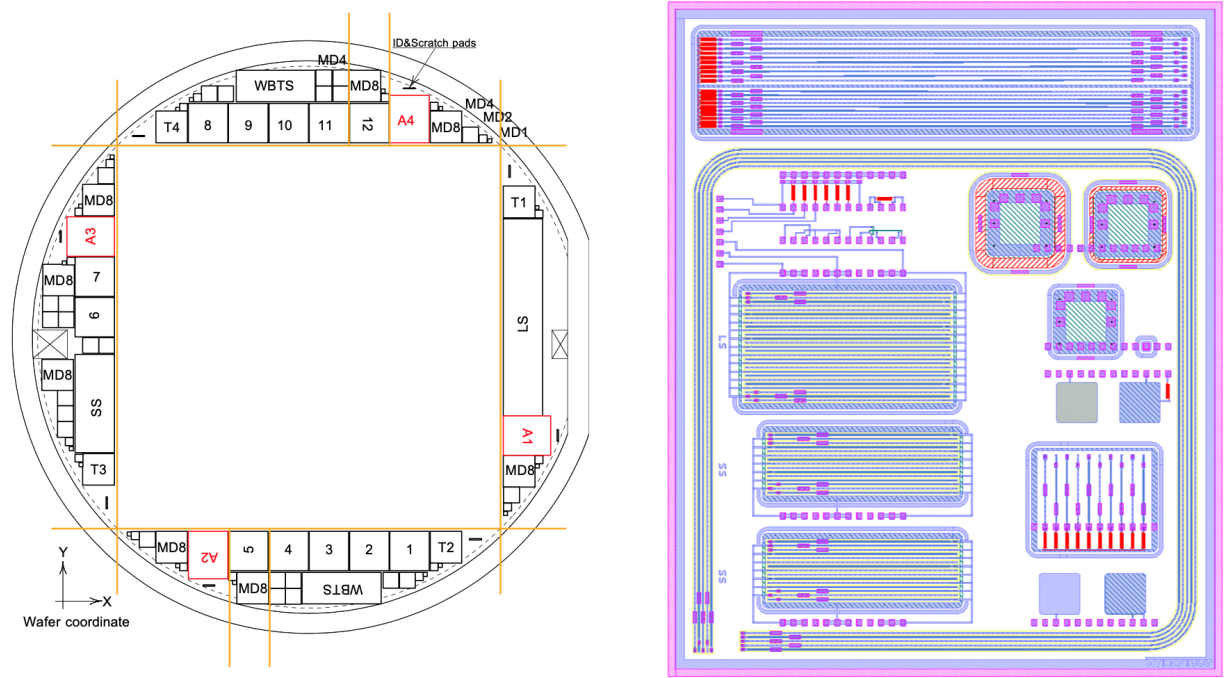

Figure 3.3: (Left) Example wafer layout of a barrel sensor demonstrating the halfmoons at the sensor periphery. Dicing lanes are denoted by yellow lines. The four ATLAS test chips are highlighted in red. (Right) Schematic of the ATLAS test chip. 


\subsubsection{Test Devices}

The manufacturing process dictates that ITk main sensors are produced from circular silicon wafers which are then diced to the appropriate sensor shape. The rounded "leftover" silicon pieces from the periphery of the wafer, referred to as halfmoons, function as convenient venues for test devices whose primary utility is quality assurance (QA). This implementation is particularly useful since the test devices, being manufactured on the same wafer, will share the same electrical characteristics as the main sensor and therefore, will reflect the most operationally relevant device parameters. The test devices, as outlined in Table 3.2, consist of three sizes of miniature strip sensors, four sizes of square diodes, and four (barrel sensor) or three (endcap sensor) identical ATLAS test chips which host a variety of test structures (see also Figure 3.3).

Examining radiation effects is one area where the test devices (especially miniature sensors) are remarkably useful. In particular, altering of the sensors' electrical properties due to the high radiation of the HL-LHC cannot be reliably predicted from non-irradiated sensor characteristics. Furthermore, it is unreasonable to irradiate a high number of main sensors for the sole purpose of QA. Irradiating and subsequent testing of miniature sensors overcomes both of these obstacles by providing data on the expected sensor performance toward the end of the detector's lifetime without sacrificing main sensors.

\begin{tabular}{|c|c|}
\hline Test Device & Description \\
\hline Miniature Sensors (1-12, SS, LS) & $\begin{array}{l}\text { Similar to main sensor with reduced area. Three sizes: } 1 \mathrm{~cm} \times 1 \mathrm{~cm} \\
(\text { minis }), 1 \mathrm{~cm} \times 2.6 \mathrm{~cm}(\text { short strips }), 1 \mathrm{~cm} \times 5 \mathrm{~cm}(\text { long strips }) \text {. }\end{array}$ \\
\hline Square Diodes (MD1-MD8) & $\begin{array}{l}\text { Features an } \mathrm{n}^{+} \text {implant in the centre, a guard ring, and an edge } \\
\text { structure similar to the main sensor. Four sizes: } 8 \times 8 \mathrm{~mm}^{2} \text {, } \\
4 \times 4 \mathrm{~mm}^{2}, 2 \times 2 \mathrm{~mm}^{2}, 1 \times 1 \mathrm{~mm}^{2} \text {. }\end{array}$ \\
\hline \multicolumn{2}{|l|}{ ATLAS Test Chip (A1-A4) } \\
\hline Bias Resistor & Identical to those on the main sensor. \\
\hline PTP Structure & Identical to those on the main sensor. \\
\hline 5-Strips Structure & $\begin{array}{l}5 \text { parallel strips similar to those of the main sensor with a length of } \\
34 \mathrm{~mm} \text {. }\end{array}$ \\
\hline Coupling Capacitors & $\begin{array}{l}\text { Two total each with the same area as a } 34 \mathrm{~mm} \text { long strip. One with } \\
\text { and one without a bias resistor. }\end{array}$ \\
\hline MOS Capacitors & $\begin{array}{l}\text { Two total each with the same area as a } 34 \mathrm{~mm} \text { long strip. One with } \\
\text { and one without p-stop. }\end{array}$ \\
\hline Gated Diodes & $\begin{array}{l}\text { Two square diodes, } 2 \mathrm{~mm} \times 2 \mathrm{~mm} \text {, each with a polysilicon gate. Two } \\
\text { gate sizes: } 60 \mu \mathrm{m} \text { and } 180 \mu \mathrm{m} \text {. }\end{array}$ \\
\hline
\end{tabular}

Table 3.2: Outline of QA test devices. The test devices are all laid out on one of four halfmoons accompanying each main sensor. The ATLAS test chip includes all structures following this entry. Corresponding labels as given in Figure 3.3 (left) are also given in brackets under the "Test Device" column. 


\subsection{Physical Properties}

There are two tests to be performed on every single main sensor before any electrical probing can take place. The entire sensor surface must be imaged with a high resolution camera and the sensor surface profile must also be obtained. Descriptions of both of these procedures are detailed below. Setups specific to the Carleton site are emphasized with examples given for clarity.

\subsubsection{Metrology}

The purpose of the metrology measurement is to extract the sensor surface profile and verify the sensor bow and thickness to be within specification (see Table 3.1). This is accomplished by a 3D measuring microscope which scans across the sensor surface while using the light-section method to perform a height measurement (see Figure 3.4). In the light-section method structured light, that is light with a known pattern (a grid or horizontal bars), is emitted through a transmitter lens, reflected off an object's surface, passed through a receiver lens, and detected by a light sensor. The height of the measured surface (relative to some calibrated flat surface) is determined through triangulation based on the measured point of reflectance and the known angle between the transmitter lens and the surface.

\begin{tabular}{ll}
\hline Parameter & Specification \\
\hline \hline Keyence VR-5200 Microscope Head & \\
\hline Field of View (Horizontal $\times$ Vertical) & $24 \mathrm{~mm} \times 18 \mathrm{~mm}$ \\
Magnification Range & $\times 12$ to $\times 160$ \\
Zoom & $\times 1$ to $\times 4$ \\
Height Measurement Range & $10 \mathrm{~mm}$ without $z$-stitching or $50 \mathrm{~mm}$ with $z$-stitching \\
Height Measurement Repeatability & $0.4 \mu \mathrm{m}$ without $z$-stitching or $1 \mu \mathrm{m}$ with $z$-stitching \\
Height Measurement Accuracy & $\pm 2.5 \mu \mathrm{m}$ without $z$-stitching or $\pm 4.0 \mu \mathrm{m}$ with $z$-stitching \\
Measurable $x y$ Range & $206 \mathrm{~mm} \times 104 \mathrm{~mm}$ \\
Stage Stroke in $x y$ & $184 \mathrm{~mm} \times 88 \mathrm{~mm}$ \\
Stage Stroke in $z$ & $73 \mathrm{~mm}$ \\
Camera Element & 4 megapixel monochrome CMOS \\
Transmitter and Receiver Lens & Double-telecentric lens \\
\hline
\end{tabular}

Table 3.3: Outline of specifications for the metrology setup at the Carleton site.

At the Carleton site, the 3D measuring microscope is the "Keyence VR-5200" which has a field of view of $24 \mathrm{~mm} \times 18 \mathrm{~mm}$ (see Table 3.3). Starting from one corner of the sensor, a motorized stage scans through a grid of points (an approximately $6 \times 7$ grid is required to measure an entire sensor) in the $x-y$ plane with the microscope being triggered at each point. The images are then stitched together to get a complete and continuous profile of the sensor surface. The determination of the grid points, control of the stage, triggering of the microscope, and stitching of images is accomplished through software developed by Keyence. The setup requires the use of the "Keyence VR-5000" controller unit along with a separate PC through which the user interfaces with the Keyence software. 


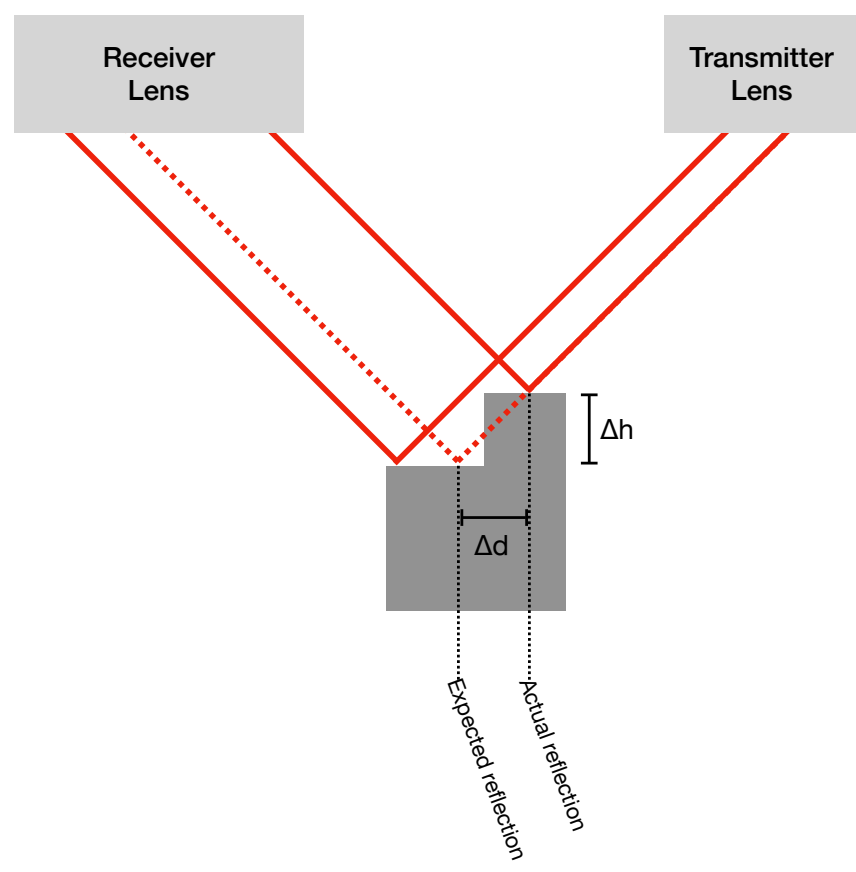

Figure 3.4: Schematic representation demonstrating the basic principle of the light-section method. In this example, $\Delta \mathrm{h}$ is determined through basic trigonometry with the known angle of transmittance and the measured displacement $\Delta \mathrm{d}$.

In order to maintain consistency in the metrology measurement output files across QC sites, a single file format has been agreed upon by all sites. A short bash script is used to convert the file output by the Keyence software into the correct format for the collaboration. This data file consists of $z$-height measurements equally spaced across the sensor surface every $10 \mathrm{~mm}$ in $x$ and $y$. The minimum $z$-height is taken as the sensor thickness where it is assumed that the sensor thickness is consistent across the sensor and changes in $z$-height are largely due to sensor bow. This parameter is also monitored by QA sites using a micrometer to measure half-moon thickness for selected wafers (for obvious reasons, this contact method for the thickness measurement is not advisable on a main sensor). The sensor bow is then taken as the difference between the maximum and minimum $z$-heights.

\subsubsection{Image Capture}

High resolution imaging of the sensor surface is the simpler of the two physical tests but is nonetheless just as necessary. This step of the QC testing process provides a baseline reference for any visual defects in the sensor. A rendering of the Carleton site setup is shown in Figure 3.5 with specifications outlined in Table 3.4. One of the key challenges in developing such a system is that it must be robust enough to accommodate 
all sensor geometries yet fast and procedurally repeatable with relative ease. In the case of the Carleton site, a 20 megapixel (MP) camera is used with a $7.1 \mathrm{~mm} \times 8.9 \mathrm{~mm}$ field of view. In order to capture the entire sensor surface (approximately $10000 \mathrm{~mm}^{2}$ ), a high precision robotic arm holds and moves a sensor beneath the camera while scanning through a predefined grid of points (see Figure 3.6). During this process the sensor is held secure on a flat plate using vacuum. A constant pitch of $8.6 \mathrm{~mm}(6.8 \mathrm{~mm})$ is used for the robot's motion in the $x$-direction ( $y$-direction). This provides $0.3 \mathrm{~mm}$ of overlap between neighbouring images. Between movement of the robotic arm and triggering of the camera at a specified grid location, a user-defined waiting period allows time for any vibrations caused by the robot's motion to settle. Because every individual main sensor passing through the QC sites must be imaged, the system developed for this purpose must do so with relative quickness. This system can successfully image the entire surface of the largest sensor in the 15 minutes allotted by the Carleton site's schedule for this QC task. Further details of the grid sizes and number of pictures required to capture each sensor geometry are outlined in Table 3.5.

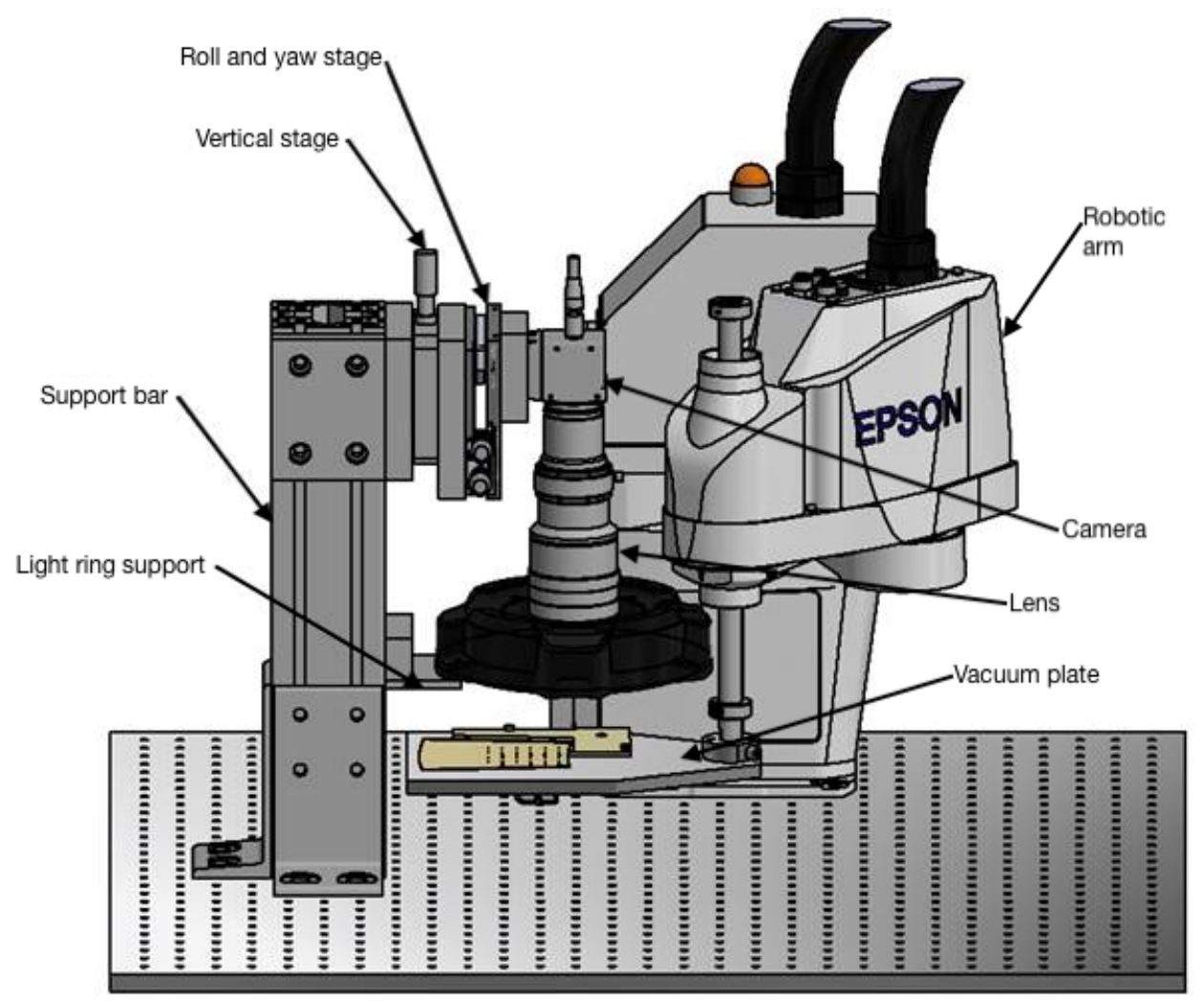

Figure 3.5: Rendering of the image capture setup used at the Carleton site. The robotic arm moves the sensor below the fixed camera. Micrometer adjustable stages are used to mount the camera to the support bar. This ensures the camera can be properly aligned and parallel to the sensor. 


\begin{tabular}{|c|c|}
\hline Parameter & Specification \\
\hline \multicolumn{2}{|l|}{ Epson Robotic Arm } \\
\hline Arm Reach in $x y$-plane $(\mathrm{J} 1+\mathrm{J} 2)$ & $400 \mathrm{~mm}(225 \mathrm{~mm}+175 \mathrm{~mm})$ \\
\hline Range in $z$-axis (J3) & $150 \mathrm{~mm}$ \\
\hline Movement Repeatability (J1,J2,J3,J4) & $\left( \pm 0.02 \mathrm{~mm}, \pm 0.02 \mathrm{~mm}, \pm 0.02 \mathrm{~mm}, \pm 0.02^{\circ}\right)$ \\
\hline Maximum Translation Speed $(\mathrm{J} 1+\mathrm{J} 2)$ & $3700 \mathrm{~mm} / \mathrm{s}$ \\
\hline Maximum Translation Speed (J3) & $1000 \mathrm{~mm} / \mathrm{s}$ \\
\hline Maximum Rotational Speed (J4) & $2600^{\circ} / \mathrm{s}$ \\
\hline Inertia (Maximum/Rated) & $0.01 / 0.003 \mathrm{~kg} \cdot \mathrm{m}^{2}$ \\
\hline \multicolumn{2}{|l|}{ Camera (CA-H2100M) } \\
\hline Image Element & $\begin{array}{l}\text { Monochrome CMOS square-pixel with unit cell size } \\
3.5 \mu \mathrm{m} \times 3.5 \mu \mathrm{m}\end{array}$ \\
\hline Pixel Count (Horizontal $\times$ Vertical) & $20.89 \mathrm{M}$ pixels $(5104 \times 4092)$ \\
\hline Electronic Shutter Speed & $0.05 \mathrm{~ms}$ to $9000 \mathrm{~ms}$ \\
\hline \multicolumn{2}{|l|}{ Lens (CA-LMH20) } \\
\hline Optical Magnification & $\times 2.0$ \\
\hline Working Distance & $80.6 \mathrm{~mm}$ \\
\hline Field of View & $8.9 \mathrm{~mm} \times 7.1 \mathrm{~mm}$ \\
\hline F-stop Range (Aperture) & 5.0 to 72 \\
\hline Resolution & $1.7 \mu \mathrm{m}$ \\
\hline Depth of Field (F-stop = 16) & $0.320 \mathrm{~mm}$ \\
\hline
\end{tabular}

Table 3.4: Outline of specifications for the image capturing setup at the Carleton site. Descriptions of working parameters for the robotic arm, camera, and lens are given. The four joints of the robotic arm are referred to as $\mathrm{J} 1-\mathrm{J} 4$. $\mathrm{J} 1$ and $\mathrm{J} 2$ translate in $x y, \mathrm{~J} 3$ translates in $z$, and $\mathrm{J} 4$ rotates about $z$.

\begin{tabular}{cccccc}
\hline Sensor & $\begin{array}{c}\text { Grid Size } \\
\mathbf{x} \times \mathbf{y}\end{array}$ & Unused Grid Points & Total Images & $\begin{array}{c}\text { Approx. Time } \\
\text { per Sensor (min) }\end{array}$ & $\begin{array}{c}\text { Approx. Storage } \\
\text { per Sensor (GB) }\end{array}$ \\
\hline LS \& SS & $12 \times 15$ & 0 & 180 & 12 & 3.6 \\
R0 & $12 \times 16$ & 10 & 182 & 12.1 & 3.64 \\
R1 & $14 \times 13$ & 7 & 175 & 11.6 & 3.5 \\
R2 & $8 \times 19$ & 1 & 151 & 10.1 & 3.02 \\
R3 & $9 \times 18$ & 1 & 161 & 10.7 & 3.22 \\
R4 & $11 \times 17$ & 13 & 174 & 11.6 & 3.48 \\
R5 & $12 \times 16$ & 7 & 185 & 12.3 & 3.7 \\
\hline
\end{tabular}

Table 3.5: Parameters for Carleton site image capture setup as shown in Figure 3.6. 

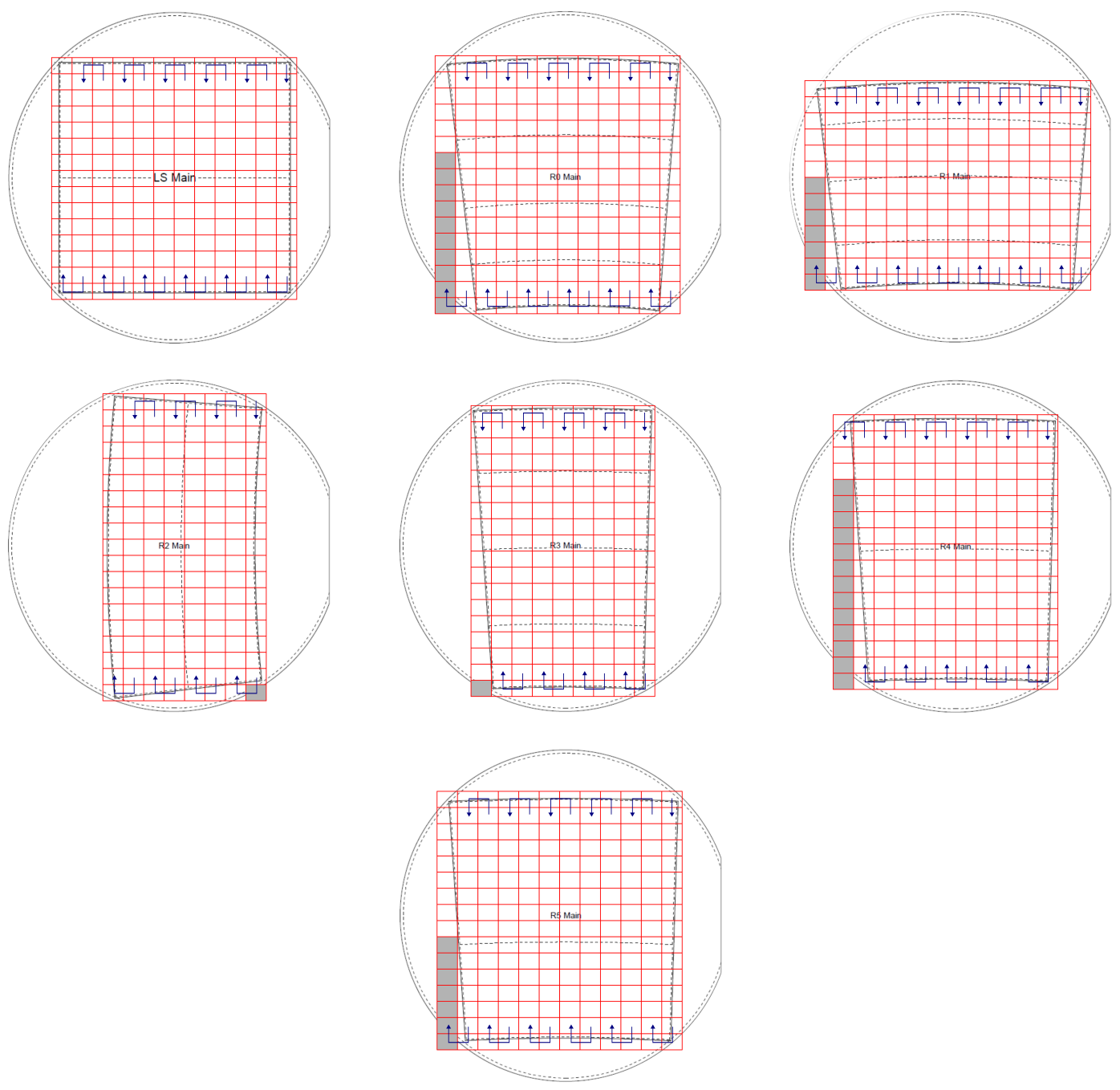

Figure 3.6: Layout of all image capture maps. Also detailed in Table 3.5.

\subsection{Electrical Tests}

The suite of electrical tests for QC are divided into two categories: tests performed on every individual sensor and those performed on a sampled basis. For every single sensor the current response and bulk capacitance are obtained as a function of bias potential. These tests performed on each individual sensor are required to validate the electrical performance of the sensor and discern whether it will eventually be built into a sensor module. For $20 \%$ of the sensors the stability of the leakage current at a set bias potential is also monitored over a 24 hour period. On a sampled basis (5\% of sensors) characteristics such as strip current, coupling capacitance, bias resistance, interstrip properties, and the PTP structure are measured in addition to the three tests above. The tests performed on a sample basis are intended to expose any inconsistencies in the manufacturing process from one batch of sensors to another. 


\subsubsection{IV/CV Characteristics}

The measurement of the sensor's current response as a function of reverse bias potential is called the IV (current-voltage). Its primary function is to determine at what voltage, up to some maximum, the sensor experiences the early onset of micro-discharge or breakdown. This voltage is designated as the sensor's breakdown voltage, $V_{B D}$. The IV has the simplest of all setups requiring one high voltage source and measure unit (SMU). The SMU should have resolution in the picoamp range. Passivation openings on the bias rail and edge metal allow biasing via the SMU to be applied either through probe needle contact or a wire-bonding setup. Increasing reverse bias is applied in $10 \mathrm{~V}$ steps and after a 10 second settling delay, the current is recorded at every step.

The measurement of the bulk capacitance of the sensor, again as a function of bias potential, is known as the $\mathrm{CV}$ (capacitance-voltage). As larger and larger reverse bias is applied the depletion region grows and the bulk capacitance will decrease with a $1 / C^{2}$ relationship until some voltage threshold. At this threshold the sensor is fully depleted such that the bulk capacitance remains constant even with increasing bias. This setup requires a single SMU as with the IV but with the addition of an LCR (inductance, capacitance, resistance) meter in parallel which measures the bulk capacitance at each 10Vbias step. A plot of the inverse capacitance squared as a function of bias potential allows the full depletion voltage to be extracted with relative ease by applying two linear fits, one sloped and one horizontal. The full depletion voltage, $V_{F D}$, is then given by the intersection of the sloped and horizontal lines

The test circuits for the IV/CV characterization along with sample measurements are shown in Figure 3.7. In both cases the sensor is modelled by a diode in parallel to a series RC (resistance-capacitance) circuit. The RC circuit includes the resistance of the undepleted bulk $\left(R_{\text {undepl }}\right)$, the bulk capacitance $\left(C_{b}\right)$, and the resistance of all bias resistors in parallel $\left(R_{a b i p}\right)$. The circuit for the IV is straightforward, but it is the basis for biasing which is used in every other QC test. In both cases, an additional resistor is added in the bias line in order to prevent large, potentially damaging currents from flowing through the circuit (there is no need to risk compromising the test equipment). A $2.2 \mu \mathrm{F}$ capacitor sits in line with the LCR meter, again to protect against any large current spikes. This circuit protection capacitor has little influence on the measurement since it is orders of magnitude larger than the bulk capacitance of the sensor (ie. the bulk capacitance dominates since they are in series). Furthermore, since it is constant, it can be accounted for either during analysis or by the LCR meter itself ${ }^{2}$.

The three examples are shown specifically to highlight the features which the IV (bottom left of figure) and CV (bottom right of figure) are used to identify. In the case of the IV, one sensor demonstrates a clear early onset of micro-discharge at $420 \mathrm{~V}$. Another sensor sees a gradual increase beginning at $500 \mathrm{~V}$ with breakdown at $650 \mathrm{~V}$. There is no indication of breakdown (or significant increase in current draw) in the third sensor up to $700 \mathrm{~V}$. In these cases, two sensors pass the QC criteria of $V_{B D}>500 \mathrm{~V}$ given by the hashed line.The $\mathrm{CV}$ plots demonstrate a difference in full depletion voltage between two different groups of

\footnotetext{
${ }^{2}$ Many modern LCR meters have a "trim" feature which allows the user to make a measurement of any capacitances or resistances intrinsic to the circuit. These are then appropriately subtracted away when the device under test is included in the circuit.
} 
sensors. The first two sensors reach full depletion at $210 \mathrm{~V}$ and the third sensor reaches full depletion at, or just before, $350 \mathrm{~V}$. Therefore, the first two sensors clearly meet the QC standard of $V_{F D}<350 \mathrm{~V}$ while the third sensor sits right at this limit.
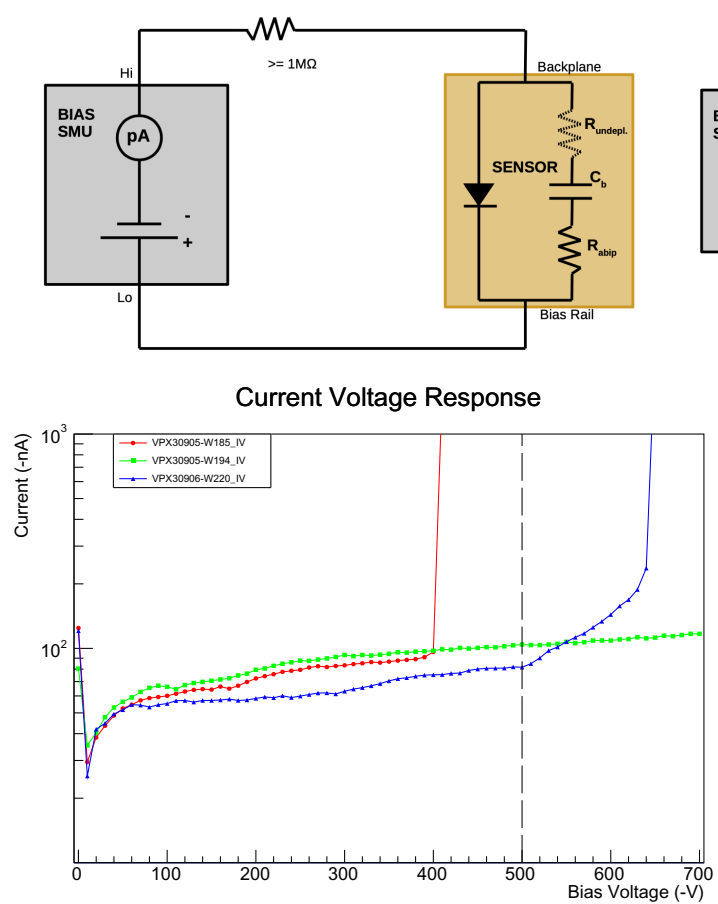

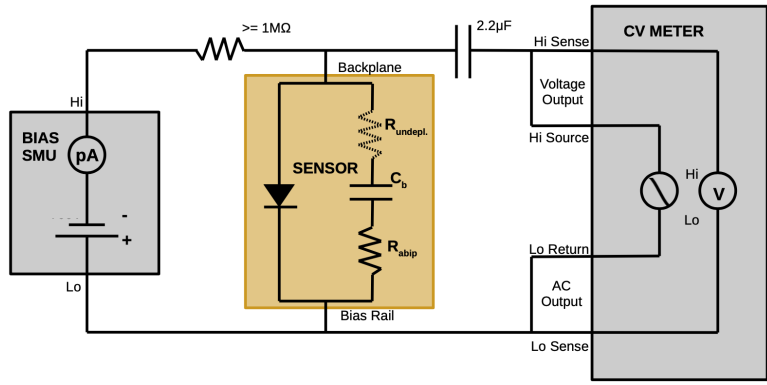

Bulk Capacitance

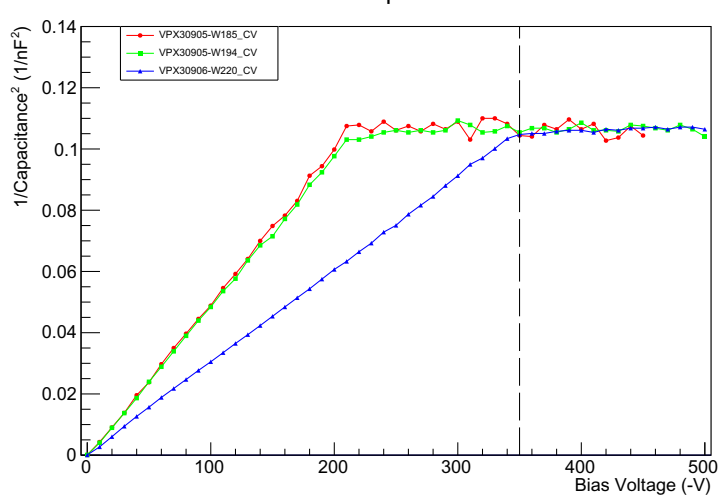

Figure 3.7: Circuit diagrams for the IV (top left) and CV (top right) test [23]. (Bottom left) Example IVs of three sensors demonstrating three different behaviours: early and immediate breakdown, late and gradual breakdown, no breakdown. (Bottom right) Example CVs of the same three sensors. One sensor reaches full depletion much later than the other two. QC limits are given by hashed lines.

\subsubsection{Stability}

The leakage current stability test is a measurement of the sensor's current draw over a 40 hour period in a temperature and humidity controlled environment. The circuit is identical to the IV test but instead of ramping up the bias voltage, the sensor is held at a constant $450 \mathrm{~V}$. There should be less than $15 \%$ variation in the temperature corrected leakage current over the entire period, and the relative humidity should be kept below $10 \%$ for the duration of the test.

\subsubsection{Full Strip Test}

The full strip test is comprised of three separate measurements made on every individual strip of a sensor while it is held at $150 \mathrm{~V}$ bias. The circuit for the strip test is shown in Figure 3.8. The three quantities obtained are strip current, coupling capacitance, and bias resistance. A high voltage SMU (separate from 
the bias SMU) applies $10 \mathrm{~V}$ then $100 \mathrm{~V}$ to the AC pad of a strip to check the current through the dielectric between the strip implant and the strip metal. In the normal case this should be $<10$ nA with a threshold of 200nA indicating a pinhole through the dielectric. The remaining two measurements of coupling capacitance and bias resistance are obtained simultaneously by an LCR meter. In this setup, the LCR meter sources a $0.1 \mathrm{~V}, 1 \mathrm{kHz}$ AC signal and assumes a series RC circuit between the strip's AC pad and the bias rail.

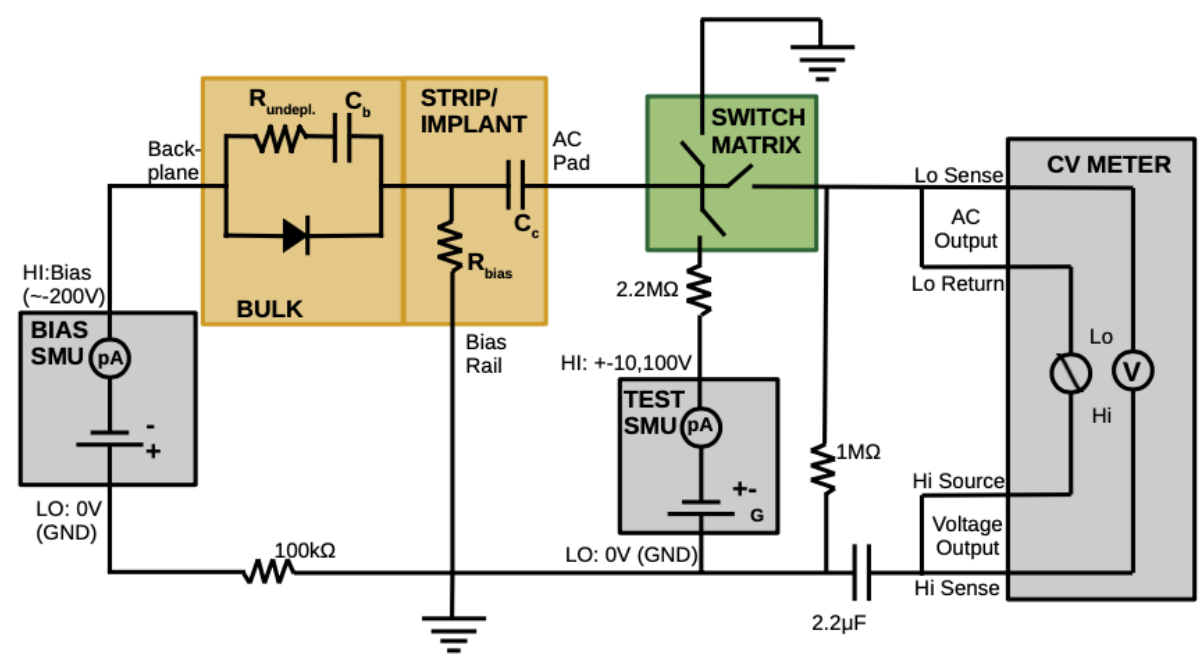

Figure 3.8: Circuit diagram for the full strip test including the optional switch matrix. [23]

\subsubsection{Interstrip Resistance}

The interstrip resistance, $R_{i s}$ measurement requires two SMUs and a high impedance voltmeter (or ammeter). One SMU is required for biasing the sensor, as usual, while the second SMU and voltmeter are used to extract the measurement. As shown in Figure 3.9, the test SMU is used to source $\pm 5 \mathrm{~V}$ to the two outer strips in $1 \mathrm{~V}$ increments while the voltmeter monitors the potential drop across the central strip. The voltmeter will see very little potential drop for values of $R_{i s}$ much higher than the bias resistance, $R_{\text {bias }}$. This measurement ensures that good strip isolation is achieved. If the interstrip isolation is compromised, then the potential drop across the central strip will tend toward the potential of the test SMU.

The following analysis of the circuit outlines exactly how a value of $R_{i s}$ is extracted. First, notice that the equivalent resistance measured by the test $\operatorname{SMU}\left(R_{s m u}\right)$ is:

$$
\begin{aligned}
\frac{1}{R_{\text {smu }}} & =\frac{2}{R_{\text {bias }}}+\frac{1}{\frac{1}{2} R_{\text {is }}+R_{\text {bias }}} \\
R_{\text {smu }} & =\frac{\left(\frac{1}{2} R_{\text {is }}+R_{\text {bias }}\right) R_{\text {bias }}}{R_{\text {is }}+3 R_{\text {bias }}}
\end{aligned}
$$

and this is, of course, just the ratio $V_{s m u} / I_{s m u}$. The first of which is sourced by the test SMU, and the 


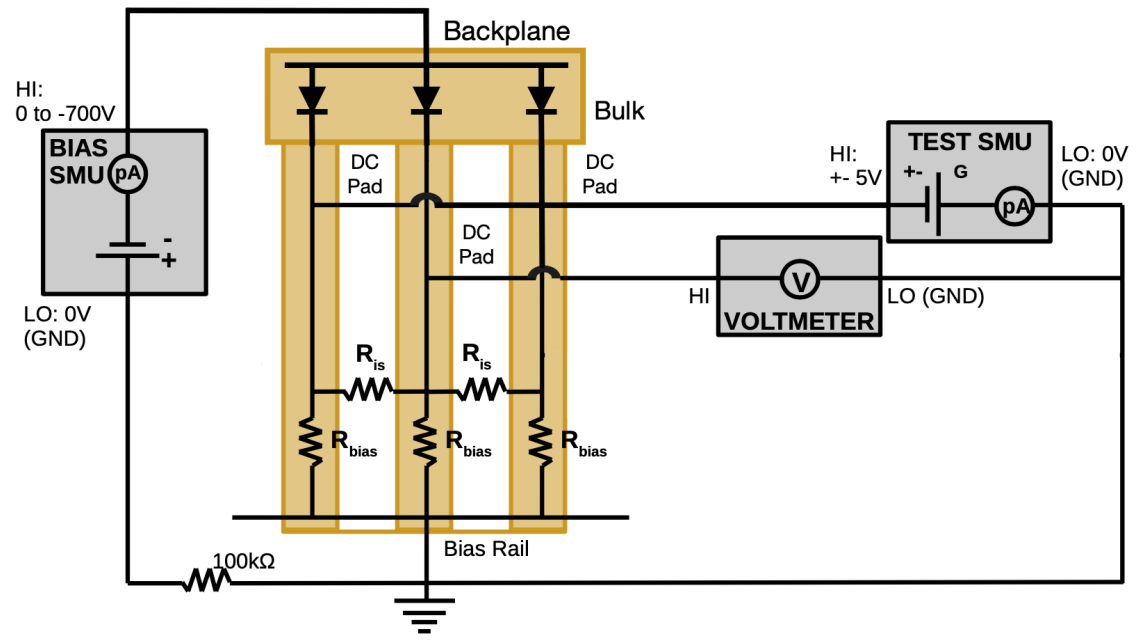

Figure 3.9: Circuit diagram for the interstrip resistance. Adapted from [23].

second of which is measured directly by the test SMU. Now, consider the following two loops of the circuit outlined in red (Figure 3.10).
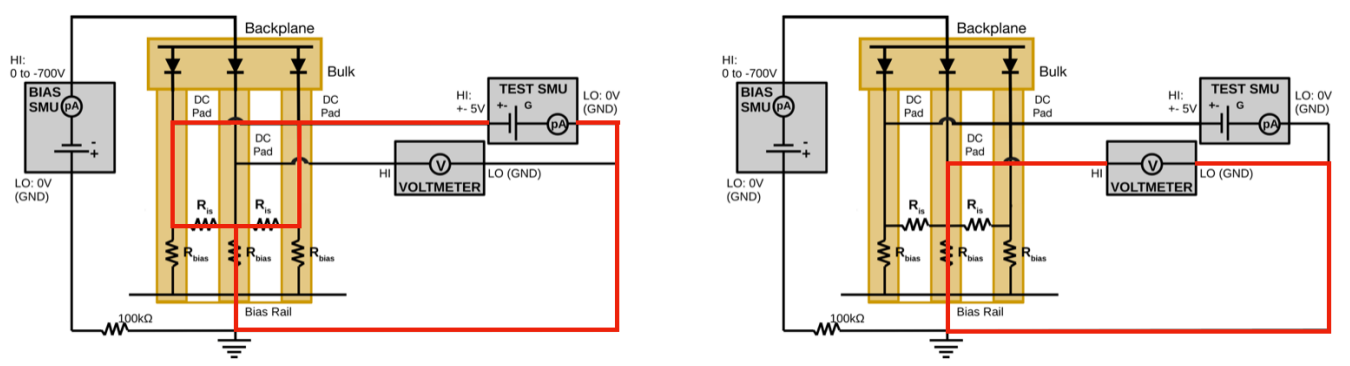

Figure 3.10: (Left) The first loop corresponding to equation 3.2. (Right) The loop corresponding to equation 3.3. Adapted from [23].

One can apply Kirchoff's loop rule in each case to find:

$$
V_{s m u}=\left(R_{\text {bias }}+\frac{1}{2} R_{i s}\right) i
$$

and:

$$
\begin{aligned}
V_{v m} & =R_{\text {bias }} i \\
i & =\frac{V_{v m}}{R_{\text {bias }}}
\end{aligned}
$$


where $i$ is the current through the middle strip. Substituting equation 3.3 into equation 3.2 gives:

$$
\begin{aligned}
V_{\text {smu }} & =\left(R_{\text {bias }}+\frac{1}{2} R_{\text {is }}\right) \frac{V_{v m}}{R_{\text {bias }}} \\
\frac{R_{\text {is }}}{R_{\text {bias }}} & =2\left(\frac{V_{\text {smu }}}{V_{v m}}-1\right)
\end{aligned}
$$

This allows $R_{\text {bias }}$ to be written as a multiple of $R_{i s}$ :

$$
R_{\text {bias }}=\beta R_{\text {is }} ; \beta=\left[2\left(\frac{V_{\text {smu }}}{V_{v m}}-1\right)\right]^{-1}
$$

Rewriting equation 3.1 and substituting in equation 3.5 gives:

$$
R_{i s}=\frac{1+3 \beta}{\frac{1}{2} \beta+\beta^{2}} R_{s m u}
$$

Thus, $R_{i s}$ and $R_{b i a s}$ are expressed in terms of measured quantities.

\subsubsection{Interstrip Capacitance}

Another important interstrip property is interstrip capacitance. This quantity contributes to the overall noise seen by the readout electronics and so should be as small as possible. As with the $R_{i s}$ measurement, and shown in Figure 3.11, a three strip setup is implemented. The strips' AC pads are probed with an LCR meter. With the central strip connected to the low output of the LCR meter and the two outer strips connected to the high end, a $0.1 \mathrm{~V}, 100 \mathrm{kHz}$ AC signal is sourced in order to directly measure the value of the interstrip capacitance. It is important that the resolution of the LCR meter is in the sub-picofarad range as the expected interstrip capacitance is of order $1 \mathrm{pF}$.

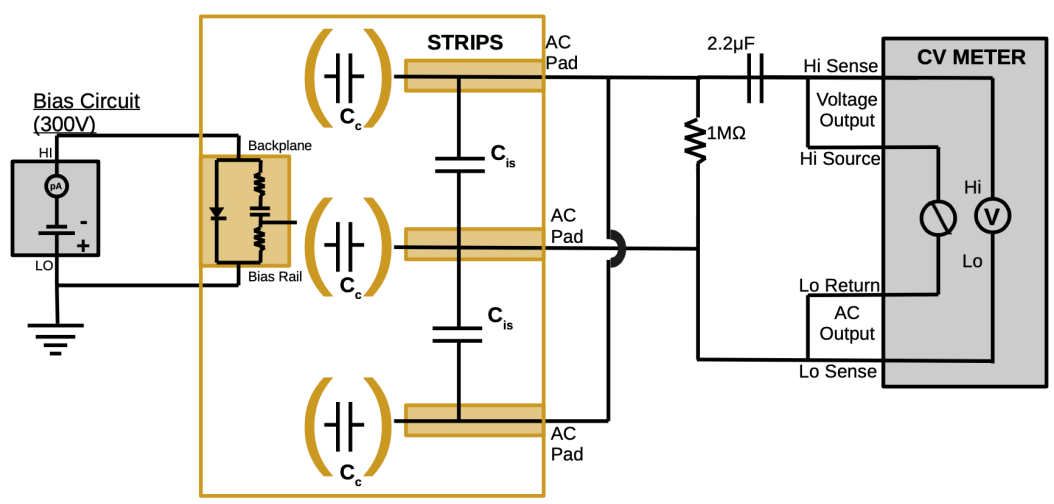

Figure 3.11: Circuit diagram for interstrip capacitance. [23] 


\subsubsection{PTP}

Punch-through-protection (PTP) structures have been implemented to protect the front-end readout electronics from any large inrush of current. The PTP structure acts as a gate between the strip implant and the bias rail so that, given a large enough voltage, the current is directed to ground via the bias rail. Figure 3.12 shows the test circuit needed to verify these PTP structures. The test requires the application of a potential between a strip implant (via the DC pad) and the bias rail using an SMU sourcing 0-40V in $1 \mathrm{~V}$ steps. This is done while the sensor is under bias to maintain strip isolation. The current measured as a function of voltage allows for examination of the onset voltage of the PTP gate. While the gate is closed, the PTP resistance will be much higher than the bias resistance and so the measured resistance (slope of the test SMU's IV curve) will be indicative of the bias resistance. After some voltage threshold, the gate will open and the measured resistance between the implant and the bias rail will decrease significantly.

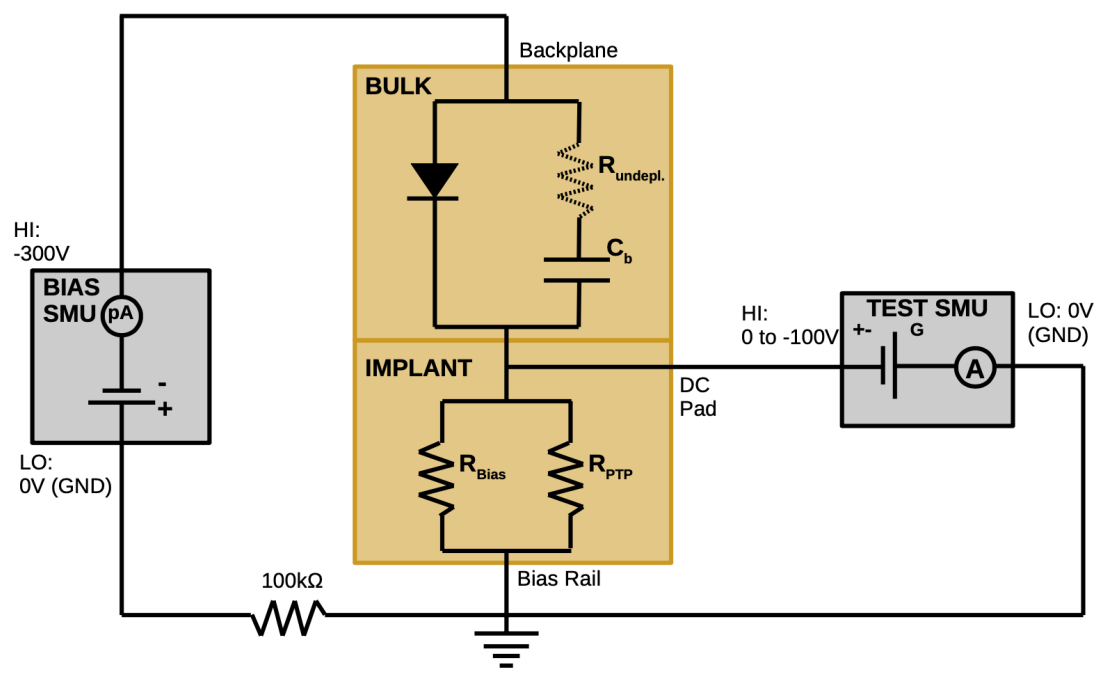

Figure 3.12: Circuit diagram for PTP. [23] 


\section{Chapter 4}

\section{Results for Prototype Sensors}

This chapter will give an outline of the analysis used to extract QC parameters for the tests described in chapter 3. In some cases, the analysis is trivial since the desired quantity is measured directly. Where this isn't the case, more detail is given to describe the process of extracting the desired parameters. The description of analyses is accompanied by results from a selection of prototype sensors. In particular, there are three groups of sensors for which results are presented. The first group are of batch 2 ATLAS12 R0 endcap sensors, the second group are of ATLAS17 LS barrel sensors, and the third group are of batch 3 ATLAS12 R0 endcap sensors. These sensors are the final iterations of prototyping before the design is ultimately solidified for preproduction and production. Further characterizations of the ATLAS12 and ATLAS17 sensors are in [24],[25],[26],[27].

\subsection{Physical Properties}

\subsubsection{Metrology Results}

Metrology results on a sample of eight ATLAS12 R0 sensors are shown in Figure 4.1. These sensors all belong to the third batch of ATLAS12 sensors. The measured thickness of each sensor lies in the range $324 \mu \mathrm{m}$ to $331 \mu \mathrm{m}$ which is within the limit specified in Table 3.1. The total bow of each sensor is also within acceptable limits with the maximum $75 \mu \mathrm{m}$ being well below the required $200 \mu \mathrm{m}$. There is a noticeable distinction between two groups in the bow measurement where three of the eight sensors have about twice as much bow as the other five. This distinction is not a cause for concern since the total bow of any one sensor is still well within the specification. It should also be noted that the relatively low statistics for this measurement is reflective of how recent the metrology setup has been implemented at the Carleton site. 

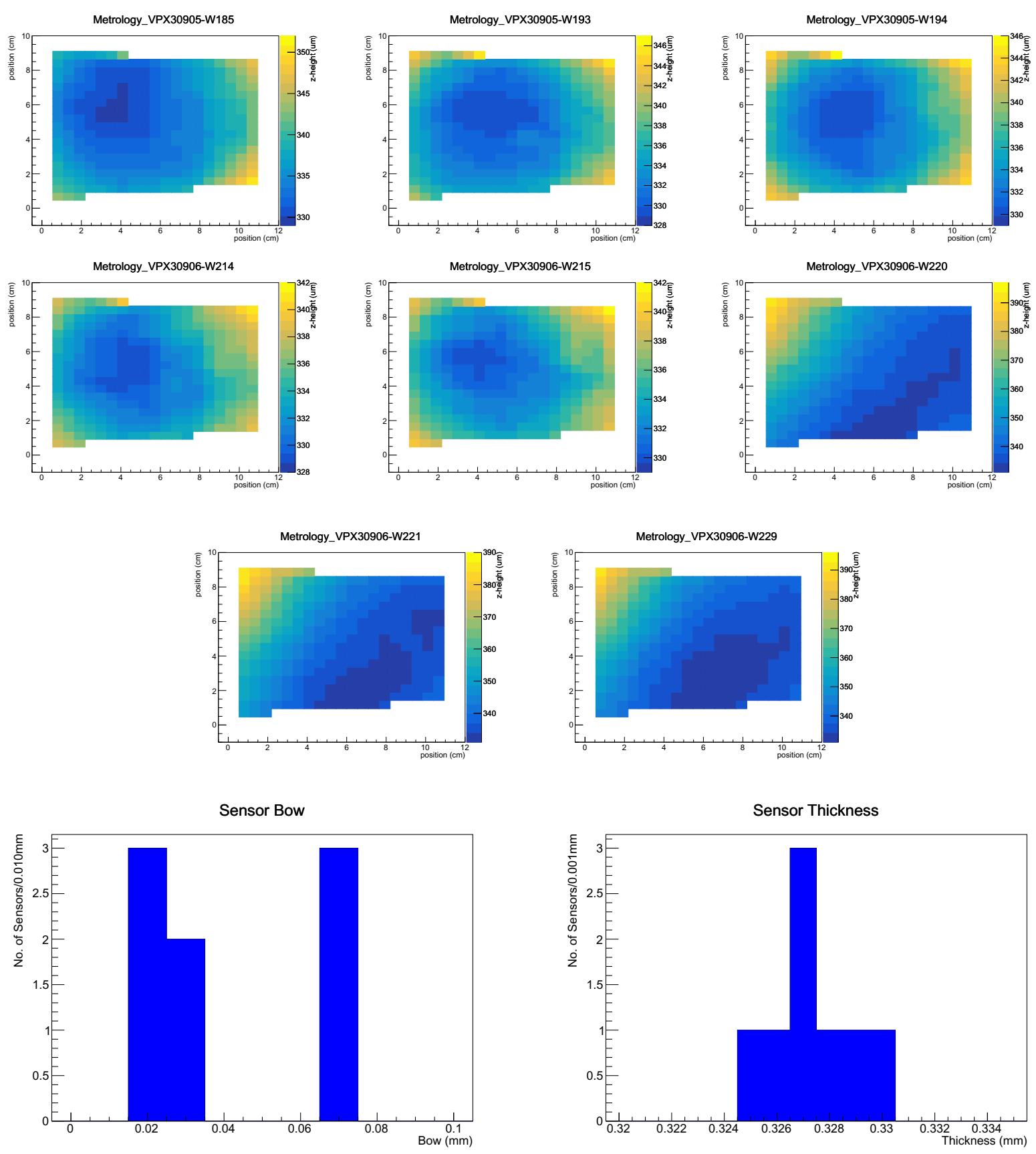

Figure 4.1: Measured sensor bow and thickness for eight batch 3 ATLAS12 R0 sensors. 


\subsection{Electrical Tests}

\subsubsection{Results}

The characteristic IV from three groups of sensors are presented (also in the next section) in Figure 4.2. These groups represent two prototype generations ATLAS main sensors. It should be noted that identifying a failed sensor in this case is not necessarily negative since many of these sensors were shared with the purpose of cross-checking the consistency of methodology and results between QC sites. The first point, at $0 \mathrm{~V}$, often reads a relatively high current. This is thought to be a result of how the Keithley 2410 sources zero volts. It is not cause for concern as this effect is swiftly eliminated as soon as a non-zero voltage is sourced to the sensor.
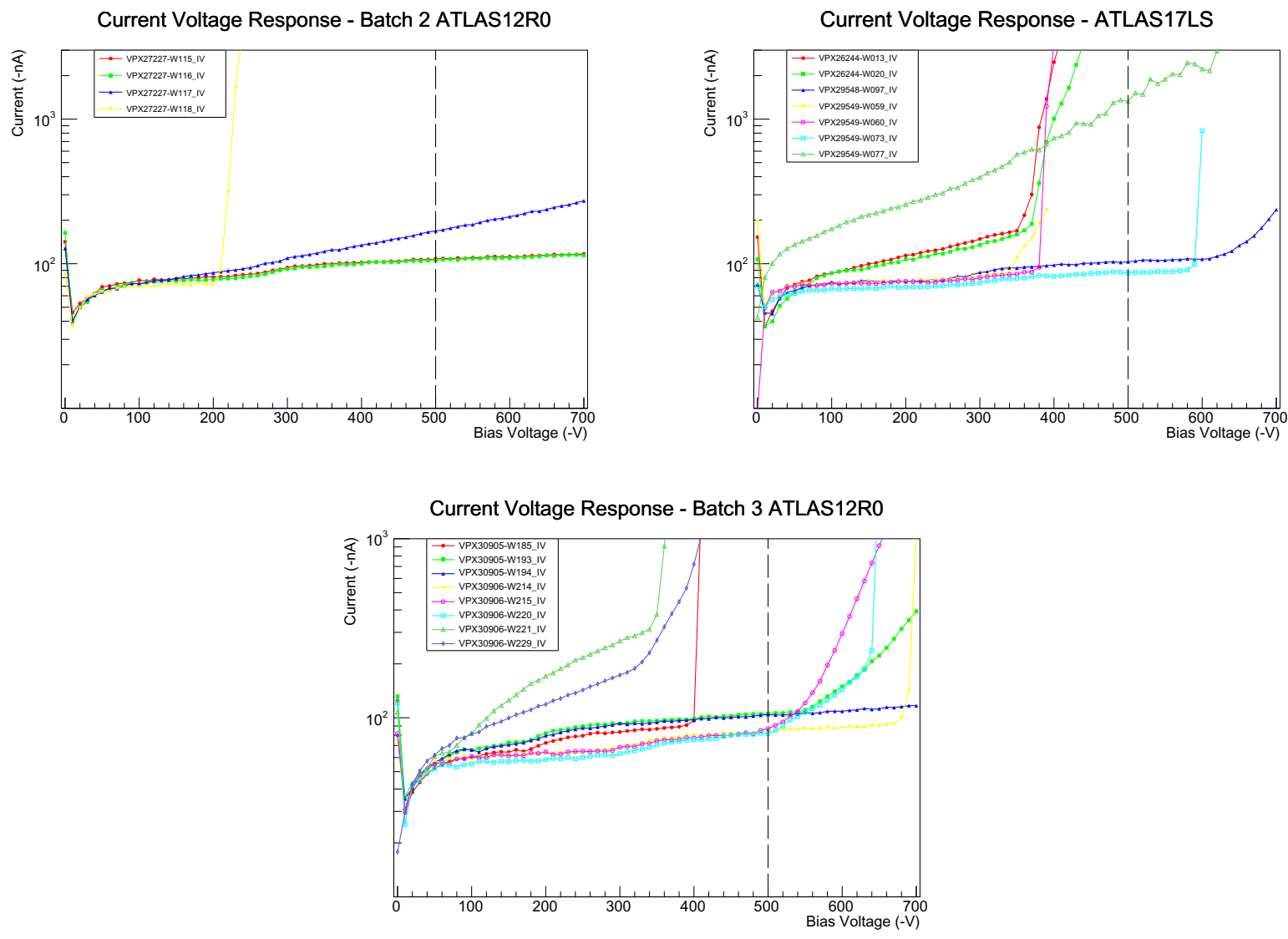

Figure 4.2: IV curves from three groupings of sensors: ATLAS12 R0 (top left), ATLAS17 LS (top right), ATLAS18 R0 (bottom left).

The first group (top left of figure) are batch 2 ATLAS12 R0 main sensors. All but one of these sensors fall within the QC limit with a well-behaved current response. In the second grouping, comprised of ATLAS17 LS barrel sensors, there are a higher number of sensors which do not meet the QC requirements. Of the failed sensors, all but one demonstrate swift and immediate breakdown. The final group consists of batch 
3 ATLAS12 R0 sensors. In this group, there is more variety in the type of breakdown observed; gradual, swift, or a combination of the two. Overall, sub-optimal performance is observed, especially in this batch of ATLAS17 LS sensors. Further investigation of sensor degradation is presented in chapter 5.

\subsubsection{CV}

Measurement of the bulk capacitance yields at least three important sensor properties. The first is the full depletion voltage which, as mentioned in the previous chapter, is given by the intersection of two linear fits applied to the $1 / C^{2}$ vs $\mathrm{V}$ plot. The collaboration has not yet finalized the algorithm used to determine this intersection point or "kink" in the CV. In this work, the suggested method is to use a combination of the Least Square Method (LSM) and a chi-squared minimization. Given CV data in the range 0V to 500V, the algorithm begins by setting the intersection point to $50 \mathrm{~V}$, well below the expected full depletion voltage. Two lines are fitted using the LSM in the ranges $0 \mathrm{~V}$ to $50 \mathrm{~V}$ and $50 \mathrm{~V}$ to $500 \mathrm{~V}$, respectively. The chi-squared value is then calculated for the total fit of each line in their respective ranges. The process is repeated as the intersection point is incremented higher until a sufficient range of values has been scanned. This gives the chi-squared value as a function of the intersection point which will be minimal when the intersection corresponds to the full depletion voltage. The other two important properties extracted from the $\mathrm{CV}$ are the depletion depth (or active thickness) and effective doping concentration. Depletion depth is given by the capacitance at full depletion using equation 2.15. The slope of the fitted $1 / C^{2}$ vs $V$ plot yields the effective doping concentration as in equation 2.17 .

Figure 4.3 gives the measured CV for the same three groups of sensors as shown in Figure 4.2. Many of the sensors with early breakdown have unstable CV curves despite the use of a current limiting resistor in series during data acquisition. In one case, sensor W118 (top left, upside down triangle), the CV is incomplete as a result of the high current draw triggering the SMU's compliance level. Of particular interest are the two subgroups clearly seen in the batch 3 R0's. The clear change in full depletion voltage between sensors with identification VPX30905 and those with VPX30906 is almost certainly indicative of a difference in the silicon manufacturing between these sensor groups.

Included in Figure 4.3 are histograms of the effective doping concentration and depletion depth at full depletion for these sensors. Since the depletion depth is basically fixed (at least it is bounded from above by the physical thickness), the effective doping concentration is the parameter largely responsible for setting the full depletion voltage of the sensor. For three sensors from the third batch of ATLAS12 R0's, the effective doping concentration is quite low resulting in a steeper slope of the CV curve. In these three cases the sensors reach full depletion well before the required $350 \mathrm{~V}$, whereas the other five sensors of this batch barely reach full depletion (much later) due to their larger effective doping concentrations (more gradual slopes in the $\mathrm{CV}$ ). The active thickness, given by the depletion depth, looks well-behaved in all sensors. Assuming physical thicknesses on the high end of accepted specification $(335 \mu \mathrm{m}$, see Table 3.1), all sensors have an active thickness $>90 \%$ of the physical thickness as required. This assumption need only be applied to those sensors whose physical thickness has not yet been measured, but for the case of the batch 3 ATLAS12 R0's 
Bulk Capacitance - Batch 2 ATLAS12R0

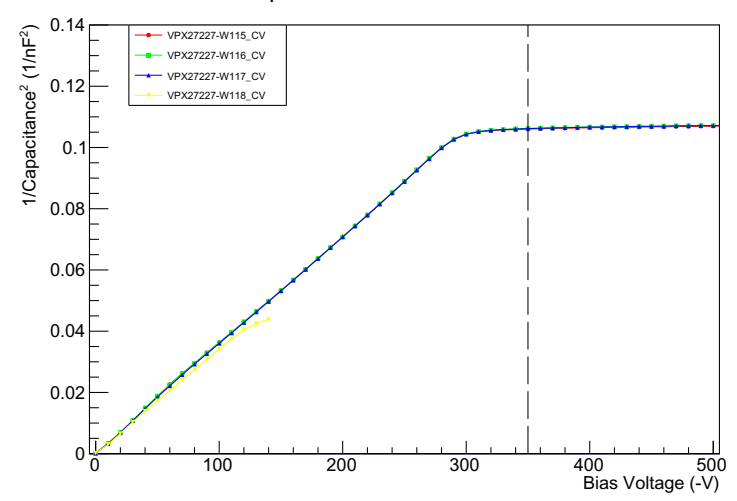

Bulk Capacitance - ATLAS17LS

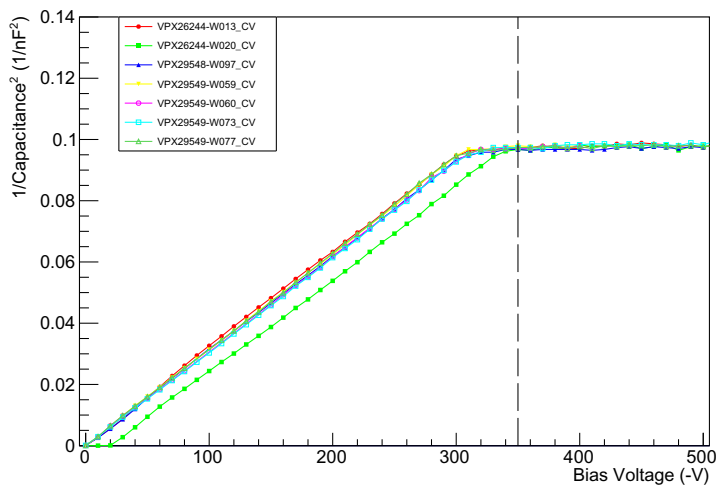

Bulk Capacitance - Batch 3 ATLAS12R0

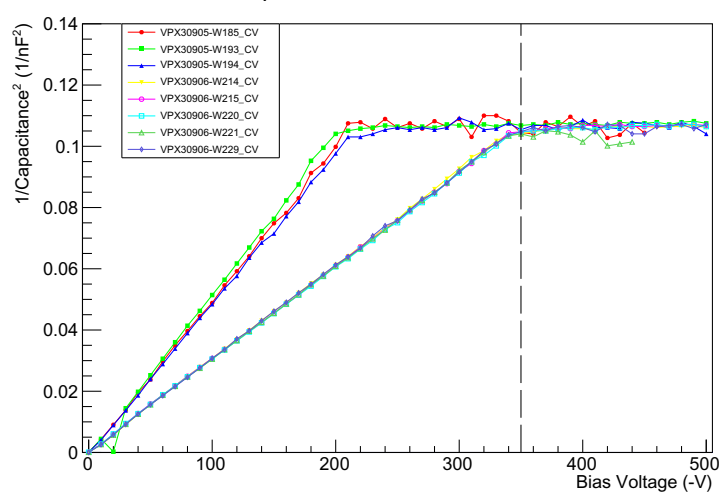

Effective Doping Concentration

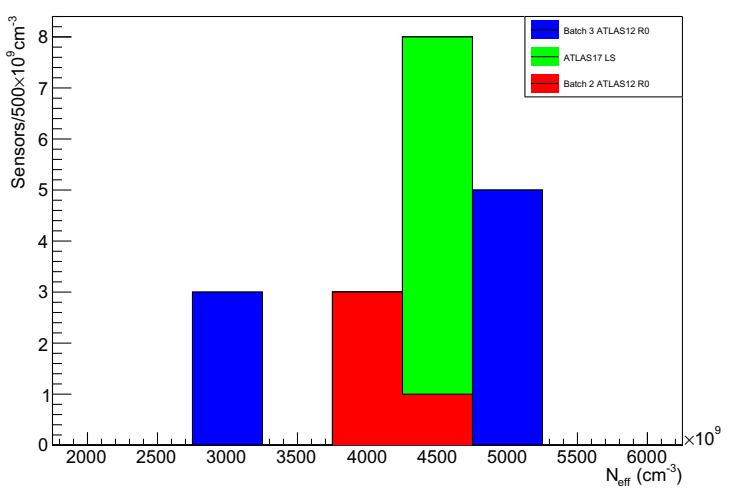

Depletion Depth

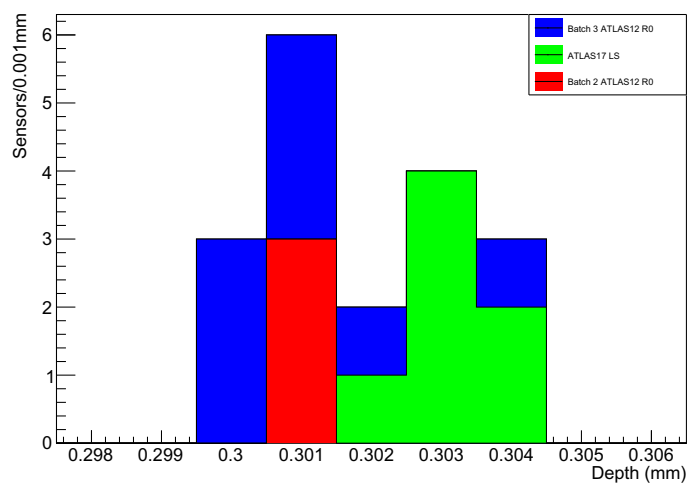

Figure 4.3: CV curves from three groupings of sensors: Batch 2 ATLAS12 R0 (top left), ATLAS17 LS (top right), Batch 3 ATLAS12 R0 (center). (Bottom left) A histogram of the effective doping concentration obtained for all sensors. (Bottom right) A histogram of the depletion depth at full depletion for all sensors. 
a comparison can be made to their measured thickness to confirm they are within specification.

\subsubsection{Striptest}

The full striptest is used to identify a variety of potential defects on a strip-by-strip basis. Although only three quantities are measured for each strip (section 3.3.3), at least five classifications of defects can be identified (Figure 4.4). The first class of defects depends only on the strip current. A strip with current above a set threshold (200nA) while $10 \mathrm{~V}$ or $100 \mathrm{~V}$ is applied is said to have a pinhole, a small electrical short between the strip implant and the top metal. Strips with low coupling capacitance but whose bias resistance is within specification (outliers included) indicate an implant break. Top metal shorts between two or more strips are identified when the coupling capacitance is increased and the bias resistance is decreased by the same factor. This multiplicative (for capacitance) or reductive (for bias resistance) factor is proportional to the number of strips whose top metal are shorted together. In the case where both coupling capacitance and bias resistance are out of specification but the defect does not fall into either of the two previous categories, the defect is identified as an open (broken) resistor. In this case, the LCR meter may also readout as an "open circuit". The final classification is broadly called "Rbias defect" since a defect is clearly present but does not fall into any of the other categories. This kind of defect can arise when there is loss of strip isolation between two or more neighbouring strips. It may also be possible for some manufacturing imperfection in the bias resistor to be present and so cause the strip to be in this category of defect.

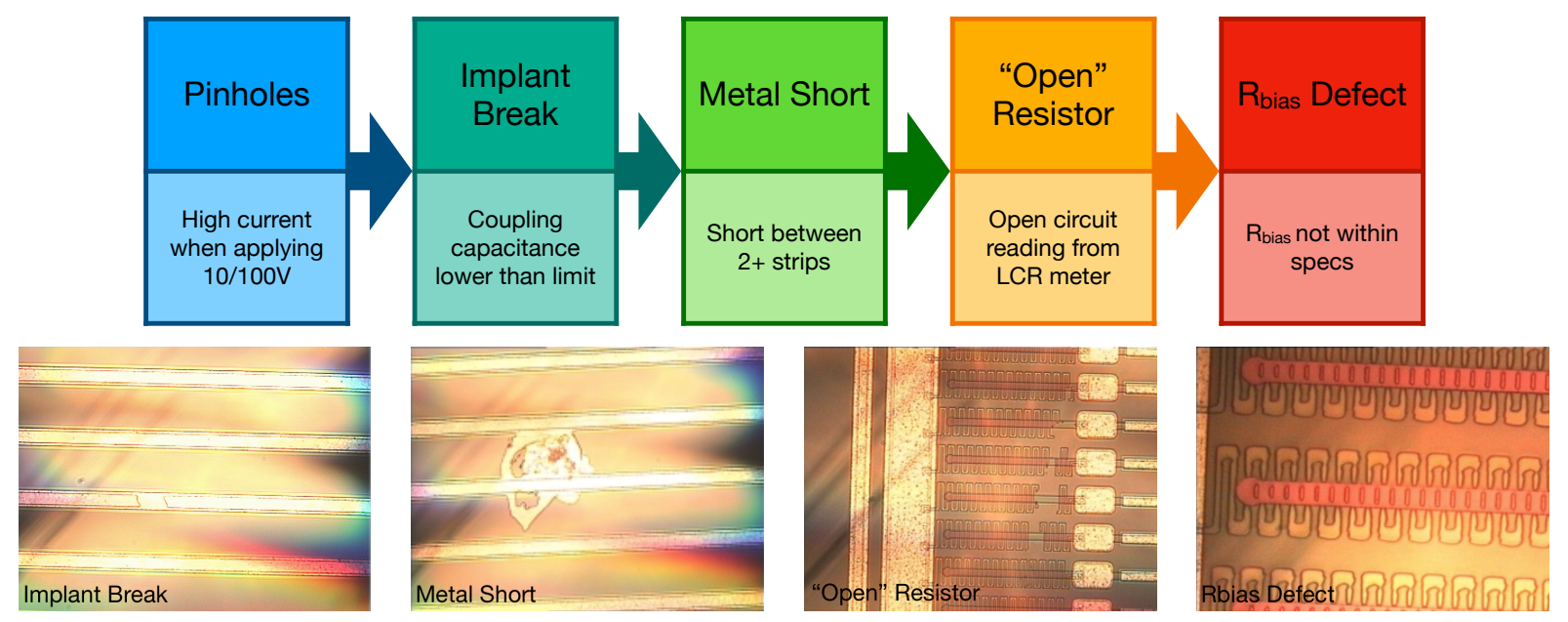

Figure 4.4: (Top) Flowchart of strip defect classification. (Bottom) Images of specific defects which may arise in the ITk strip sensor manufacturing process.[28]

Three examples are shown for the full striptest with QC limits indicated by the hashed lines. In the first case, sensor W020, every individual channel meets the requirements and so there are no failed channels identified (Figure 4.5). This sensor was further studied after some degradation was observed in the bias resistance measurement (see chapter 5). Because this is an LS barrel sensor, there are only two segments of 
strips. Additionally, the longer strips result in higher overall coupling capacitance, hence, the QC limit has been adjusted accordingly as compared to the next two examples.

The second example, sensor W194, is that of an ATLAS12 R0 (Figure 4.6). This sensor has a notable region of strips in segment 2 which have apparently very low, almost zero, bias resistance. The region is also distinguishable in the coupling capacitance measurement, however, not by a large enough amount to suspect a top metal short or an open circuit failure. The small increase to measured capacitance is likely an artifact of the LCR meter automatically changing its "range" settings in order to accommodate the lower measured resistance. The low measured bias resistance is consistent with a loss of strip isolation in this region. With low interstrip resistance the bias resistors will measure in parallel to one another and reduce by a factor equal to the number of strips contributing to the problem. Three pairs of two strips with measured bias resistance equal to exactly half of the expected value are also present to further support this notion.

The third example, sensor W214, is also an ATLAS12 R0 (Figure 4.7). This sensor performs well in its bias resistance and coupling capacitance measurements. There are three strips at the beginning of segment 3 each with about one third of the expected bias resistance which again points to loss of strip isolation. More significantly, while a large majority of channels technically pass the requirement for strip current, there is a large overall noise level observed in this measurement as compared to the previous two examples. This is indicative of a potential problem with the surface oxide which is apparently less insulating than in the previous cases. A deeper study of the surface oxide properties are examined in chapter 5.

\subsubsection{Interstrip Resistance and Capacitance}

Measurement of the interstrip properties requires great sensitivity due to the extremes at which these values fall. In the case of interstrip capacitance, this sensitivity is achieved through a high precision, sub-picofarad LCR meter.

The analysis of interstrip resistance as described in subsection 3.3.4 requires two plots be made. The first is $V_{s m u}$ against $V_{v m}$ and the second is $V_{s m u}$ against $I_{s m u}$. For each plot a linear fit is applied and the slopes are used to extract the values $\beta$ and $R_{s m u}$ (as in equations 3.5 and 3.6). Sensitivity is gained in the interstrip resistance measurement by taking multiple measurements (up to five) of the induced voltage on the central strip (and likewise the SMU current) for each voltage applied by the test SMU. The error as given in Figure 4.8 is propagated from the error of fitting those two linear plots using the LSM.

Results for measurements of interstrip properties are limited. The examples shown (Figure 4.8) serve to demonstrate the readiness of the Carleton QC site to carry out such measurements. Interstrip resistance for the two ATLAS17 LS sensors tested is well above the QC limit of $10 \times R_{\text {bias }}$. In fact, the values are much higher, on the order of tens of gigaohms, which has already been demonstrated in the ATLAS17 (and ATLAS12) sensors [24],[26]. The usefulness of the interstrip resistance measurement in identifying

problems with strip isolation is further explored in the next chapter. In the second example, interstrip capacitance for an ATLAS17 LS sensor is well below the required $<1 \mathrm{pF} / \mathrm{cm}(4.82 \mathrm{pF}$ total for an LS barrel sensor, given by the hashed line) all the way up to $700 \mathrm{~V}$. 

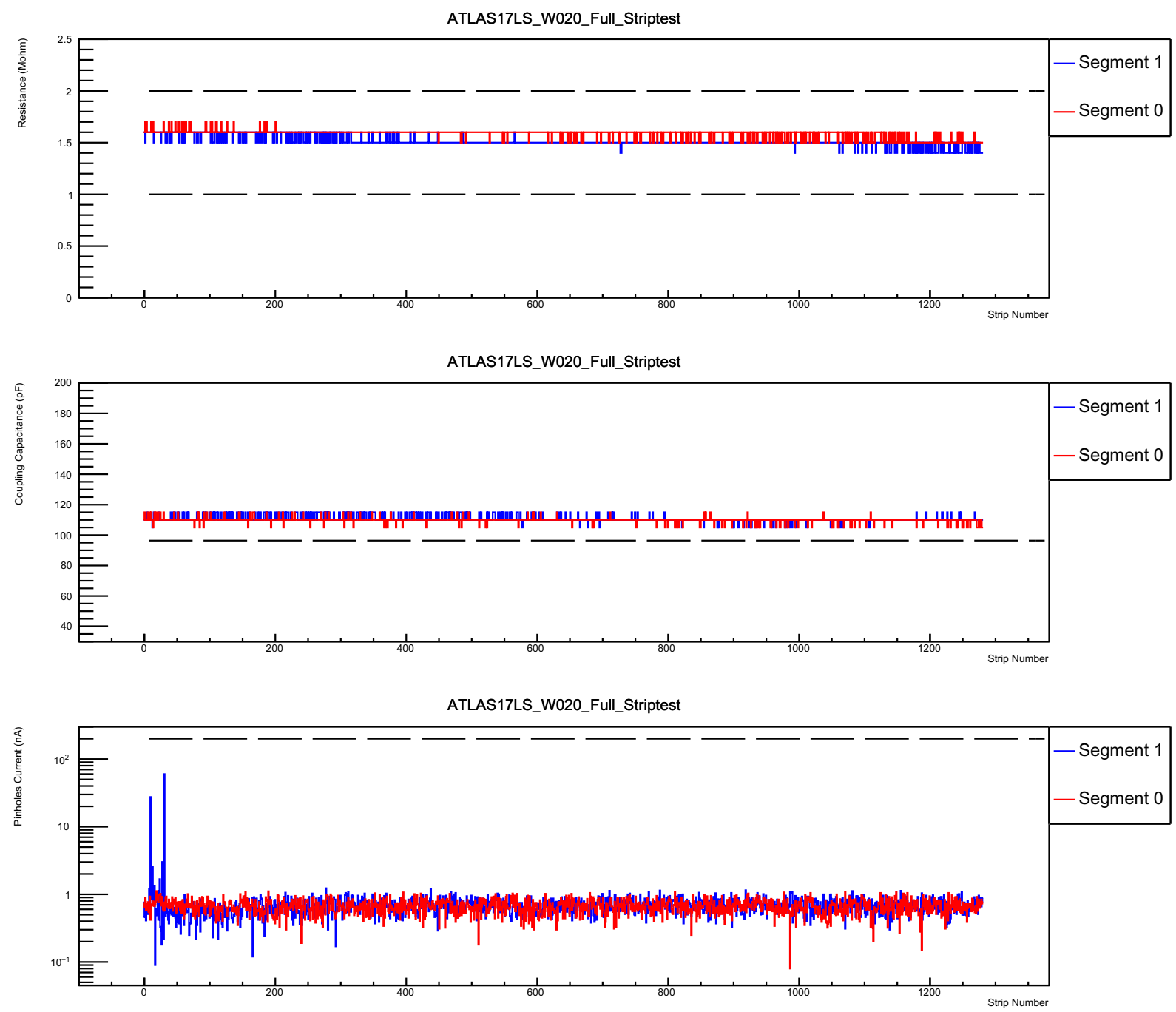

Figure 4.5: Striptest results from an ATLAS17 LS barrel sensor, W020. 

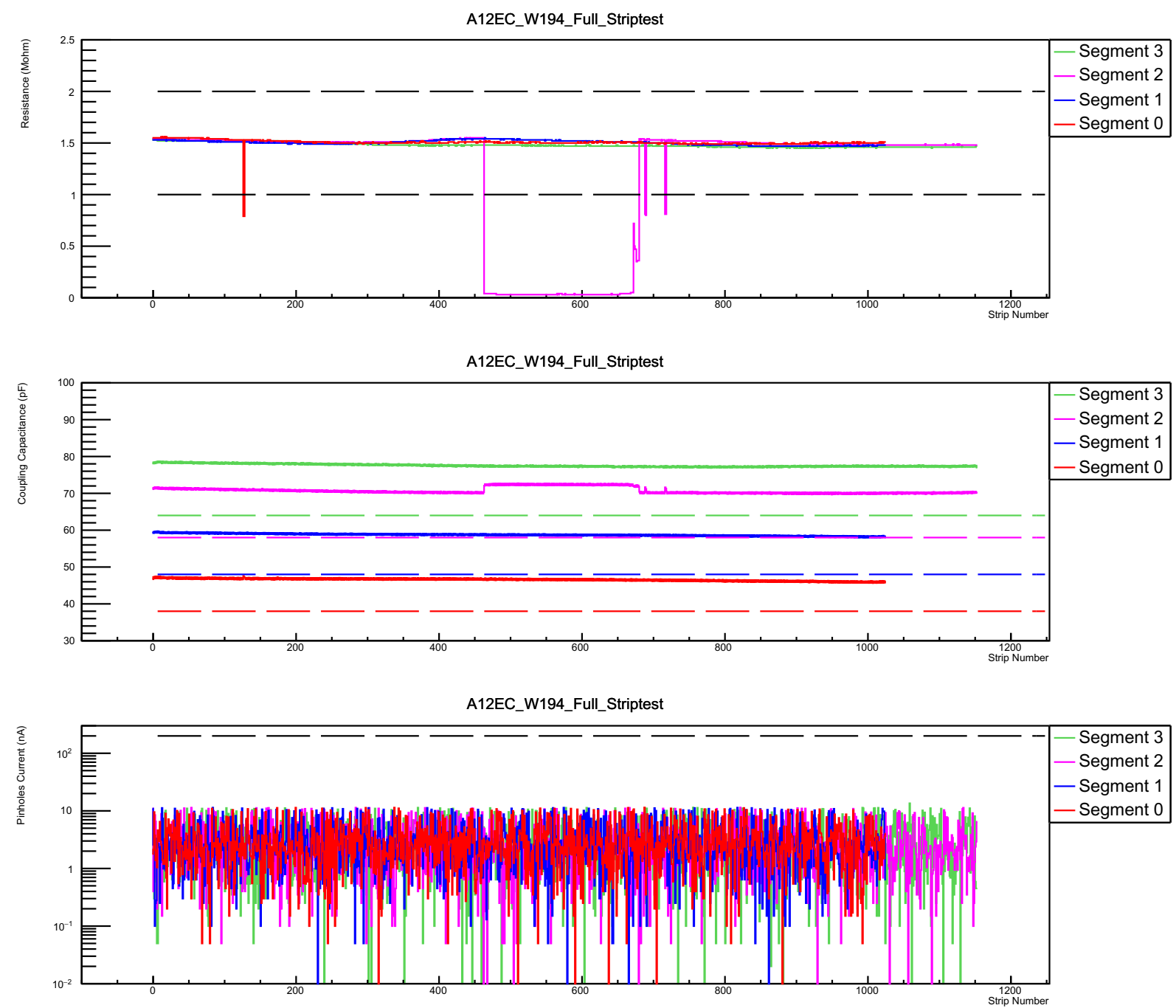

Figure 4.6: Striptest results from an ATLAS12 R0 endcap sensor, W194. 

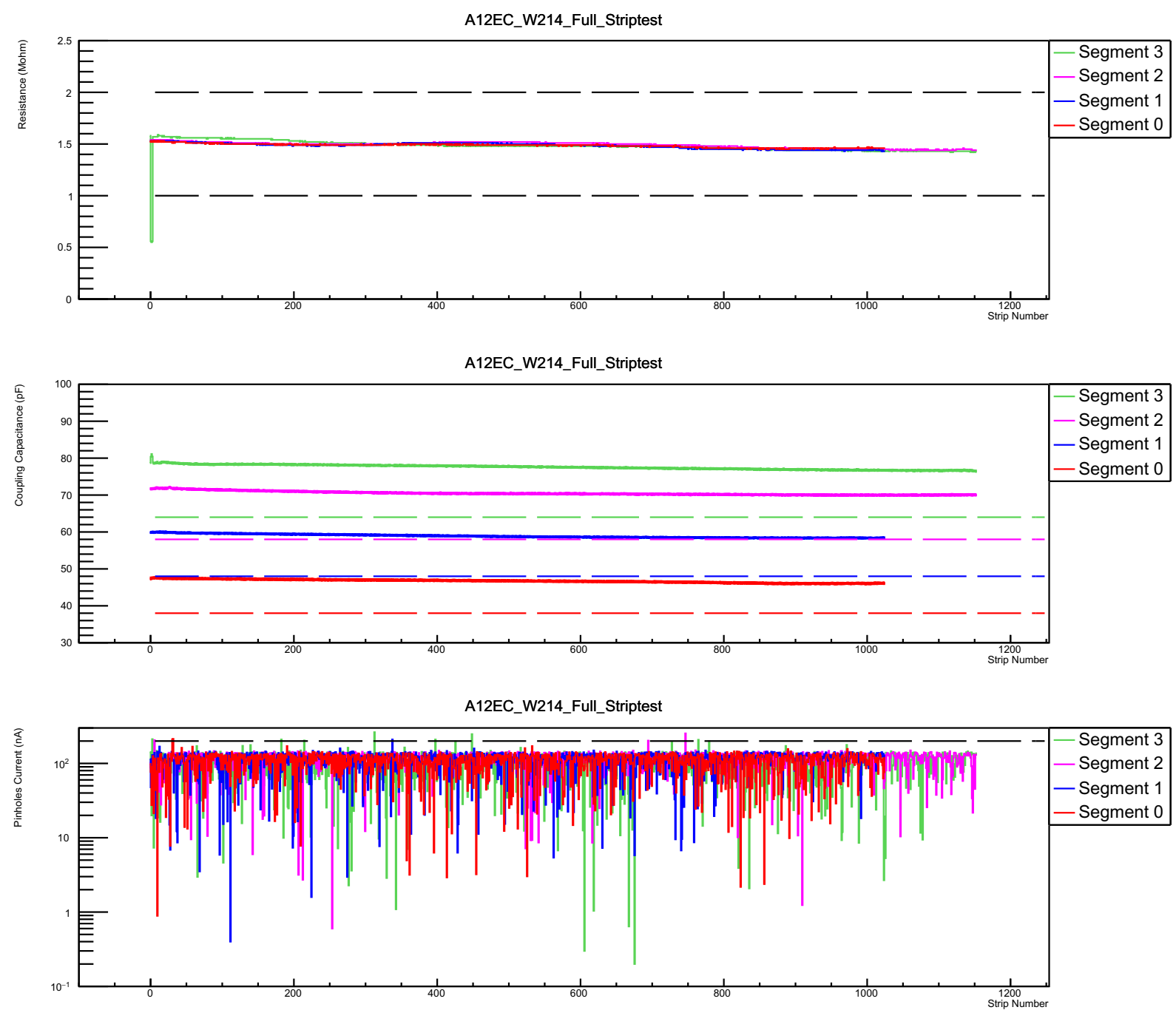

Figure 4.7: Striptest results from an ATLAS12 R0 endcap sensor, W214. 

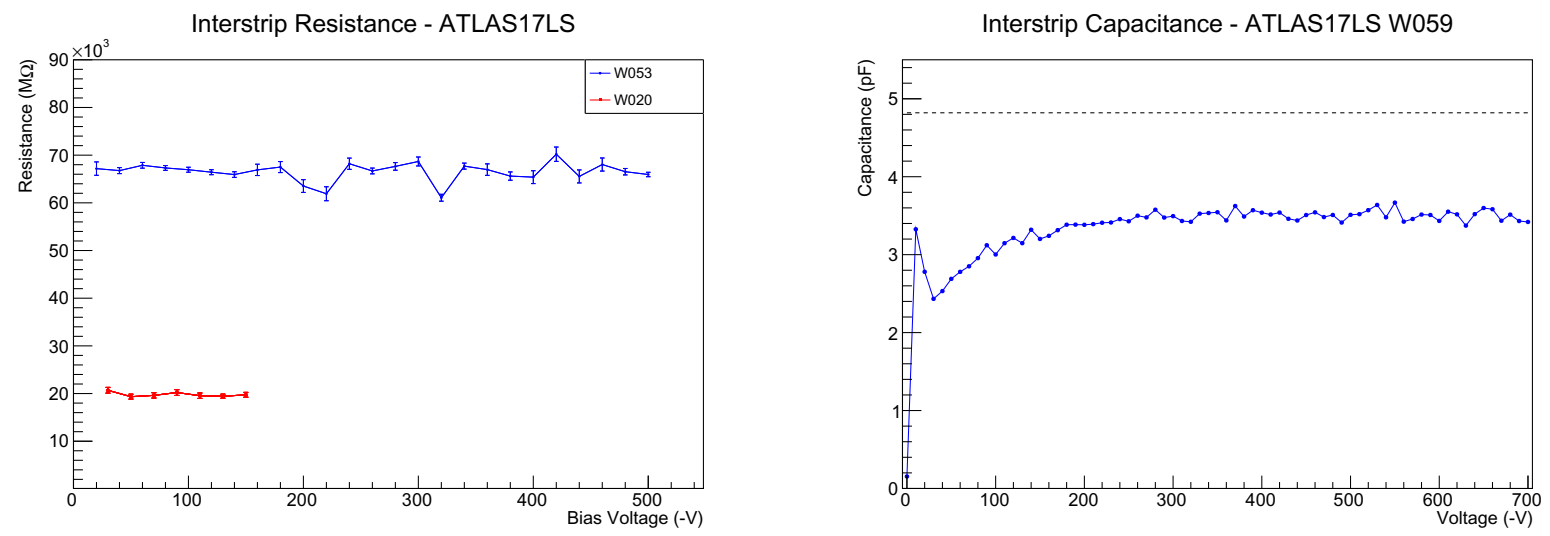

Figure 4.8: Interstrip resistance and capacitance measured against bias voltage in ATLAS17 barrel sensors. 


\section{Chapter 5}

\section{Further Investigations of Sensor Properties}

In this final chapter, three investigations beyond the scope of regular sensor QC are carried out. First, the performance of irradiated miniature sensors is examined. This examination falls in line with QA operations within the strip sensor community and holds great value in predicting the longterm performance of the ITk. Next, a case study of sensor degradation and partial recovery is presented. An attempt is made to identify the location that this degradation is most prevalent, carefully examine the sensor's test and environmental history, and finally link the degradation with prolonged exposure to high relative humidities. Finally, results of testing newly implemented MOS capacitor test structures are put forward. This work is done in part as a contribution to the ITk QA community in developing test procedures and setting acceptable limits for these structures. Beyond this goal, the MOS capacitors are further used to study field enhanced charge injection in the sensor's surface oxide.

\subsection{Performance after Irradiation}

\subsubsection{Test Setup for Irradiated Minis}

In order to achieve the appropriate conditions for testing the irradiated minis, a custom "cooling jig" was developed (Figure 5.1). The goal for the jig was to reach and maintain a temperature of $-20^{\circ} \mathrm{C}$ allowing results to be directly compared to sensor specifications (Table 3.1). Testing irradiated samples at low temperatures minimizes any annealing which is a statistically driven, temperature dependent phenomenon [21],[22],[29]. The two phase cooling scheme begins with a large copper chuck with routes for coolant milled throughout its volume. The copper serves as a good conductor of heat and is further cooled as the coolant is constantly pumped through and refrigerated. On its own, the copper chuck with coolant equilibrates around $0^{\circ} \mathrm{C}$. The second phase of cooling, between the copper chuck and aluminum enclosure, consists of two thermoelectric peltier devices. The peltiers draw heat away from the baseplate of the enclosure and deposit that heat into the large copper chuck.

One significant consideration is the relative humidity $(\mathrm{RH})$ inside the enclosure. Typical RH values in 
the test area can be as high as $45 \%$. Cooling this air down to below freezing results in condensation and the formation of frost. Gaseous nitrogen is pumped into the enclosure to dry out the air and reduce the RH to less than $2 \%$ at room temperature, which results in $\mathrm{RH}$ of less than $40 \%$ at $-20^{\circ} \mathrm{C}$.

Not used for this testing but included in the design of the cooling jig is an option to apply vacuum as a means of securing the DUT. Additionally, the copper chuck is designed to act as an adaptor to either a 6" or 8 " probe station chuck. It is foreseen that this system may be used for full sized main sensors where the use of vacuum would be beneficial to secure the sensor during testing.

Each mini is secured to a printed circuit board (PCB) via a conductive adhesive. This adhesive electrically connects the backplane of the sensor to a trace of the PCB. The bias rail is then wire-bonded to another separate PCB trace and wire leads soldered to these PCB traces. These wire leads are fed through a small opening of the aluminum enclosure and connected to an SMU for biasing. Inside the enclosure, next to the strip sensor PCB, is an identical PCB housing a temperature-humidity (TH) sensor. In this way, the TH sensor will experience the same environmental conditions as the strip sensor and can be used to monitor the conditions inside the enclosure as any testing is conducted.

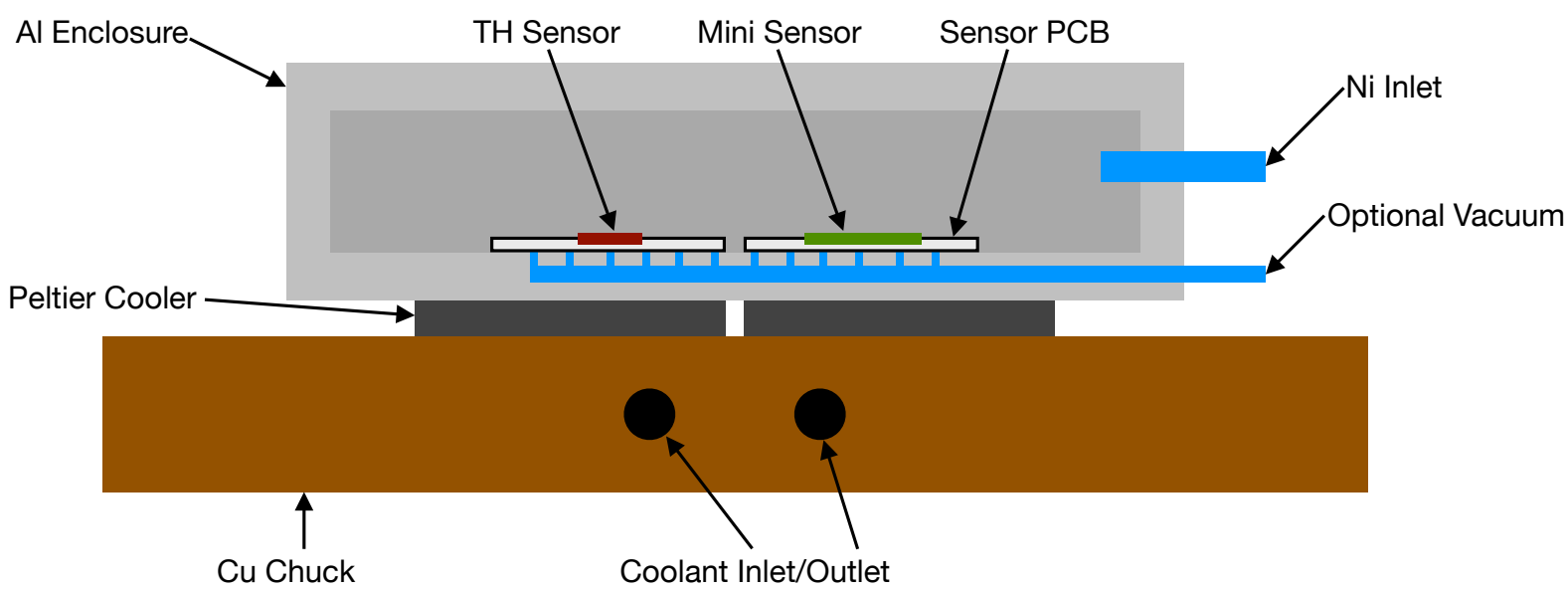

Figure 5.1: Schematic side view of the "cooling jig" setup used to test the irradiated minis. Coolant is refrigerated and pumped through the copper chuck while thermoelectric peltiers draw heat out of the aluminum enclosure and deposit that heat into the copper. Stable environments of $-20^{\circ} \mathrm{C}$ and $<35 \%$ relative humidity are achievable with this setup.

\subsubsection{Current-Voltage Response of Irradiated Minis}

The current-voltage response curves up to a bias of $-1000 \mathrm{~V}$ of 14 minis are given in Figure 5.2. These minis all belong to the same batch of ATLAS17 LS wafers and have been irradiated with protons to five fluences 
in the range $10^{14}-10^{16} n_{e q} / \mathrm{cm}^{2}$ as indicated by the legend of the figure. The irradiations of these minis were conducted at Cyclotron and RadioIsotope Center (CYRIC), Tohoku University (Japan), with a 70MeV proton beam and cooled to $-15^{\circ} \mathrm{C}$ during irradiation. Five minis total (one at each fluence) have been fully diced resulting in the "slim edge" feature identical to that of a main sensor as described in section 3.1.1. The minis were tested at $-20^{\circ} \mathrm{C}$ and $<35 \% \mathrm{RH}$ (barring one exception as indicated in the figure).

Each mini demonstrates leakage current well below the specified $100 \mu \mathrm{A} / \mathrm{cm}^{2}$ at $500 \mathrm{~V}$ even up to the highest fluence which is almost an order of magnitude higher than the required $1.2 \times 10^{15} n_{e q} / \mathrm{cm}^{2}$. A clear trend of increasing leakage current with increasing fluence is also observed as expected. There is no observed sharp breakdown of any sensor. The two sensors irradiated to the highest fluence, mini8 from W006 and mini8 from W014, exceeded the SMU's set compliance of $100 \mu \mathrm{A}$ after $860 \mathrm{~V}$ and $710 \mathrm{~V}$, respectively. The sensors with the "slim edge" do not apparently perform worse than those without the feature. Overall, this batch of sensors provide very promising indications that the total leakage current of main sensors in the ITk will not exceed expectations even at the end of the detector lifetime.

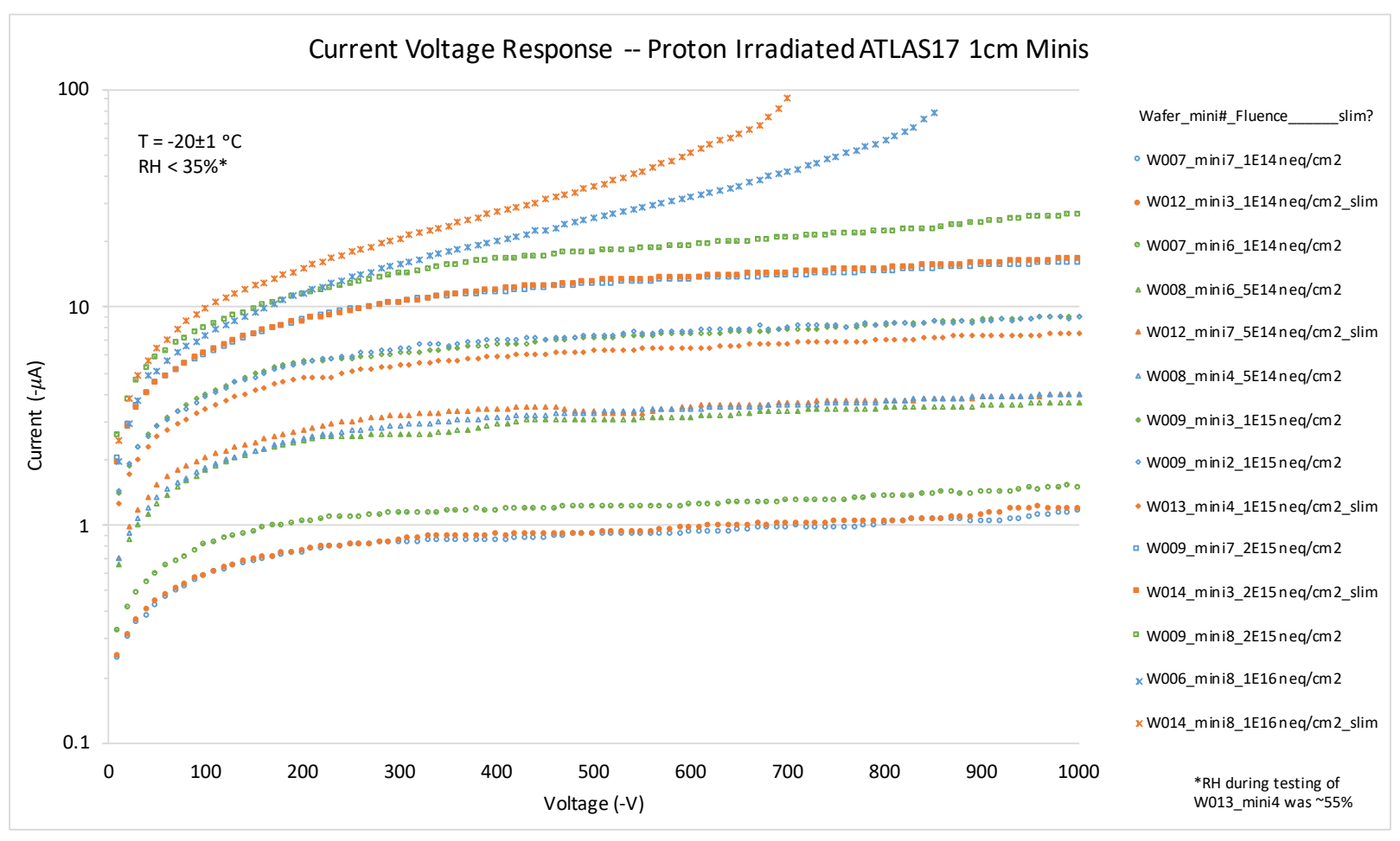

Figure 5.2: Current voltage response of 14 proton irradiated minis. The minis are irradiated to fluences $10^{14}$ (circle), $5 \times 10^{14}$ (triangle), $10^{15}$ (diamond), $2 \times 10^{15}$ (square), and $10^{16} n_{e q} / \mathrm{cm}^{2}$ (cross).

These results also qualitatively agree with those presented in [21] where the same dependence of leakage current on fluence is observed. The magnitude of the leakage currents in Figure 5.2 are overall lower which is likely due to uncertainties in the dosimetry between the irradiation sites (there can be up to $20 \%$ error in 
the calculation of equivalent proton fluence), methodology between test sites, or a combination of the two.

\subsection{Case Study: Anomalous Loss of Interstrip Isolation}

This section presents an interesting case study of sensor W020, it's observed degradation, and subsequent partial recovery. The sensor is also presented in [30] alongside two other sensors that demonstrated similar behaviour, one of which fully recovered, and two modules where high noise was observed in local regions of about 30 strips. As with the modules, an attempt is made to trace the problem with W020 back to a loss of strip isolation. The humidity sensitivity observed in the ATLAS17 sensors in [31] is suspected as the cause.

\subsubsection{Initial Sensor Performance}

The bulk sensor characteristics are given in Figure 5.3. The IV and CV were obtained by Carleton practitioners upon reception of the sensor at the Carleton site on the $13^{\text {th }}$ of June, 2018. The performance of this sensor is already sub-optimal experiencing breakdown at $370 \mathrm{~V}$. It should be noted that it is no longer common practice to bias the sensor up to $700 \mathrm{~V}$ for the $\mathrm{CV}$ since the full depletion voltage is specified to be below $350 \mathrm{~V}$ and since a new effort is being made to prevent biasing sensors beyond breakdown for prolonged periods of time. The IV and CV were performed at ambient temperature with RH around $30 \%$. After the IV and CV test, the sensor was stored in the dry cabinet with $\mathrm{RH}<5 \%$.
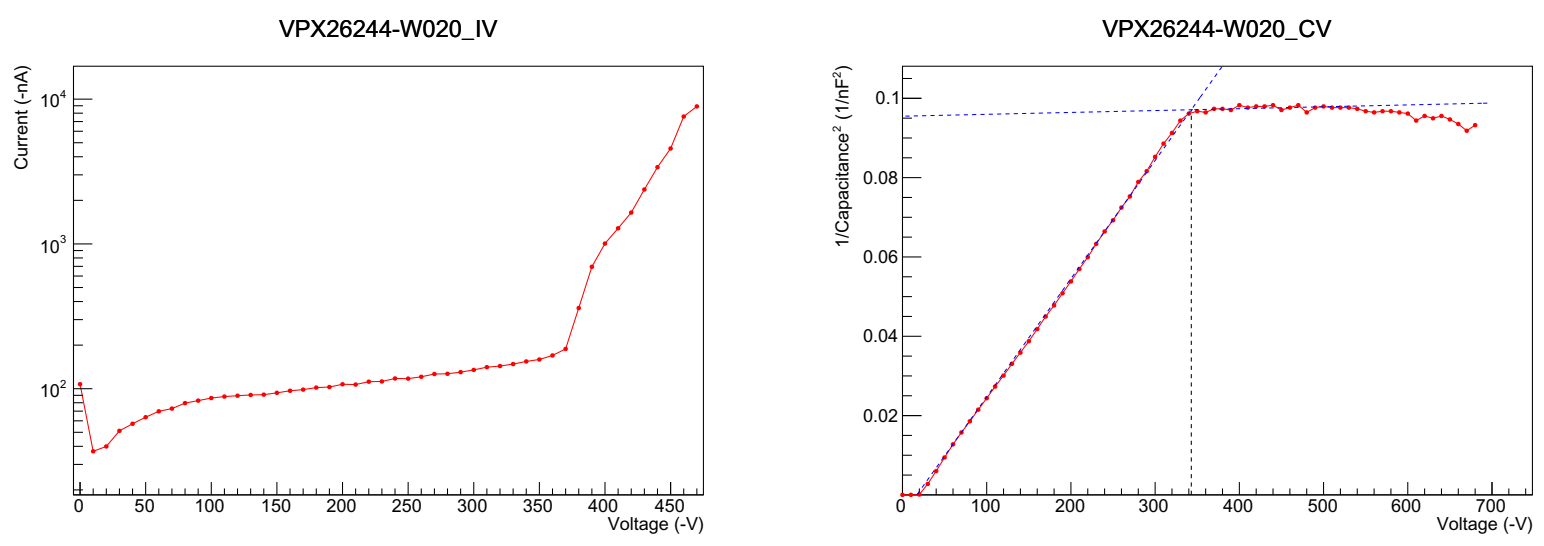

Figure 5.3: Initial sensor bulk characteristics on intake.

In the initial full strip test (see Figure 4.5) every single strip passed and no defects were identified. Upon inspection, three instances of anomalously high strip currents were observed in segment 1 . These high currents were outliers with respect to those measured on every other strip, but not high enough to pass the 200nA failure threshold. The strip test was performed on the $7^{\text {th }}$ of March, 2019, nearly 9 months after the sensor was received and the initial IV and CV obtained. The total runtime of the strip test was approximately 15.5 hours during which time the sensor was biased to $200 \mathrm{~V}$ and exposed to $\mathrm{RH}<10 \%{ }^{1}$.

\footnotetext{
${ }^{1}$ Ambient relative humidities at the Carleton site are typically quite low during winter months as a result of Ottawa weather
} 


\subsubsection{Sensor Degradation}

Almost exactly one month after the initial strip test, a second strip test was performed on the $8^{\text {th }}$ of April, 2018 (Figure 5.4, right). The key difference in this case is that the sensor was exposed to a higher relative humidity of $25 \%$ for 4 hours. The length of the test was shorter since only the resistance-capacitance measurement was performed on each strip. There are large regions of failed $R_{\text {bias }}$ in the central region of both segments with sporadic failures throughout. Notably, where failures in the $R_{\text {bias }}$ are isolated to a few consecutive strips, the measured value on each strip is approximately the nominal $1.5 \mathrm{M} \Omega$ divided by the number of strips in the sequence of failures. For example, two consecutive failures typically measure approximately $0.75-0.8 \mathrm{M} \Omega$ each and three consecutive failures measure approximately $0.5-0.6 \mathrm{M} \Omega$ each. Furthermore, there are no single isolated incidents of failure in the $R_{\text {bias }}$ measurement. These two observations corroborate the notion that the issue is not, in fact, with the bias resistors themselves but rather it is a degradation of interstrip isolation.

Following the strip-by-strip measurement of the bias resistors (and coupling capacitance) the IV was performed on this sensor again, on the $26^{\text {th }}$ of April, 2019 (Figure 5.4, left). The breakdown voltage significantly decreased to $210 \mathrm{~V}$. After this stark change in breakdown voltage, the sensor was stored in dry storage $(\mathrm{RH}<2 \%)$ and only accessed a handful of times to periodically recheck the IV for any improvements.
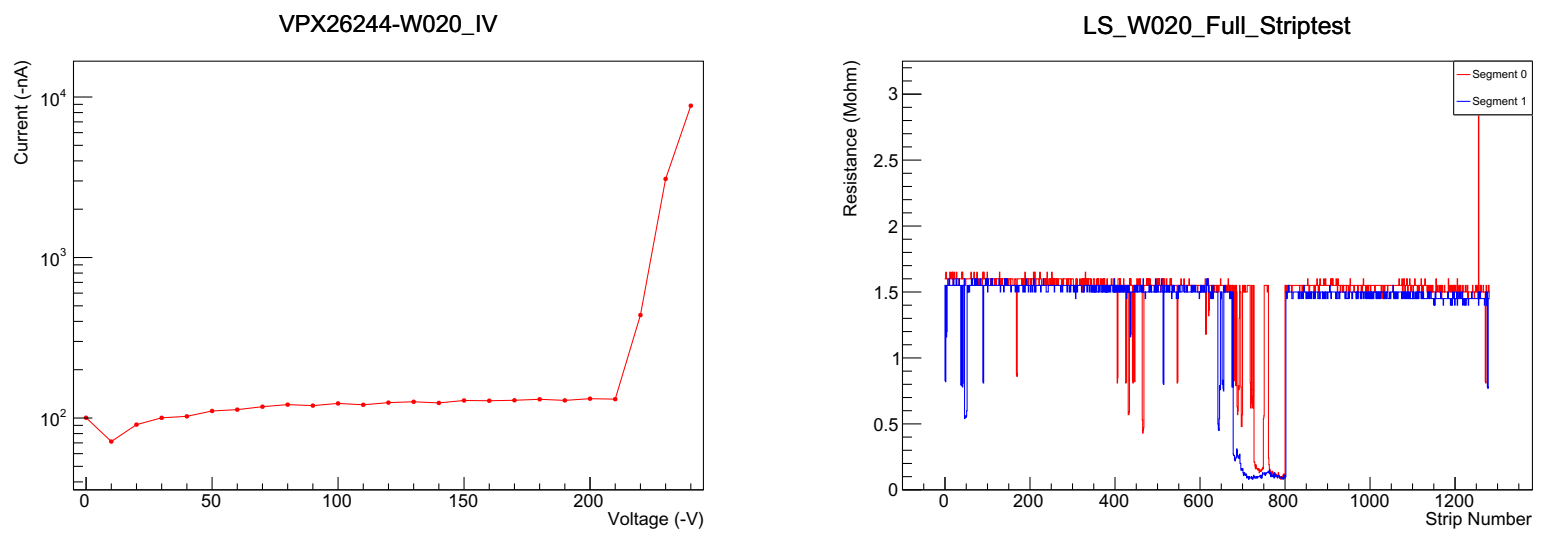

Figure 5.4: Degraded sensor performance in the IV and bias resistance strip-level test.

\subsubsection{Partial Sensor Recovery}

The final full strip test was performed on this sensor on the $20^{t h}$ of August, 2019. By this time, the sensor had spent months in dry storage and so any humidity induced effects, if reversible, were assumed to have been corrected. Because the most previous breakdown voltage of this sensor was close to the bias voltage typically used for the strip test, the sensor was only biased to $100 \mathrm{~V}$ for the duration of this final strip test. The result of the bias resistance measurement is given on the right of Figure 5.5. An apparent recovery of patterns. 
the large failed region in the centre of the sensor was observed, especially in segment 0 where only one defect was identified (this high reading of $R_{\text {bias }}$ was later confirmed to be a contact issue between the strip and probe needle).
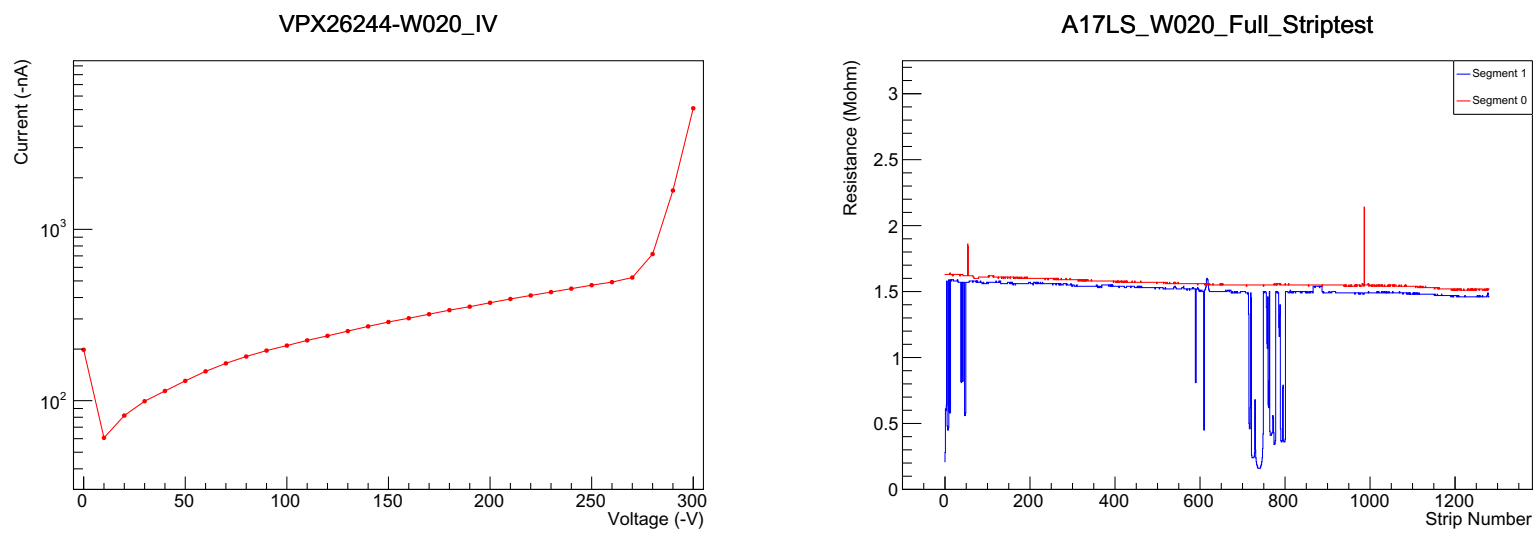

Figure 5.5: Partial recovery of sensor performance in the IV and bias resistance strip-level test.

After spending about one week in dry storage, the IV was performed again (Figure 5.5, left) but under dry conditions $(\mathrm{RH}<5 \%)$. Partial recovery of the sensor was observed with the breakdown voltage at $270 \mathrm{~V}$, but with the overall leakage current of the sensor being tens of nanoamps higher. Between the strip test and the dry IV, the sensor was wire-bonded into one of the universal ITk sensor jigs being developed at that time. It is possible that the higher overall current is an artifact of the wire-bonding operation.

\subsubsection{Examining Strip Isolation}

The interstrip resistance (Figure 5.6) was measured at two locations of segment 1 using the three probe method as described in chapter 3. Strip 600 (central strip of three probe method) was probed first. Nominal $R_{\text {bias }}$ of $1.5 \mathrm{M} \Omega$ was measured at both this strip and its neighbours (note that it is between a couple instances of failures at strips 590 and 611). Good strip isolation was confirmed with interstrip resistance of approximately $20 \mathrm{G} \Omega$ for bias potentials in the range $30-150 \mathrm{~V}$ (left of figure). The second strip probed for isolation was strip 730 which had yielded an $R_{\text {bias }}$ of $0.28 \mathrm{M} \Omega$ in the strip test measurement. The interstrip resistance measurement in this case confirmed poor strip isolation (right of figure).

The low interstrip resistance measured in this region provides an explanation to the failures in $R_{\text {bias }}$. The strip test assumes good strip isolation. Without this isolation, the parallel resistance of neighbouring strips will start to have a non-negligible impact on the $R_{\text {bias }}$ measurment. Essentially, with very low interstrip resistance the bias resistors of neighbouring strips will act in parallel to one another and reduce the overall resistance measured by the LCR meter. The coupling capacitance will remain unchanged, however, since there is still only the capacitance between one strip implant and top metal in the circuit. 

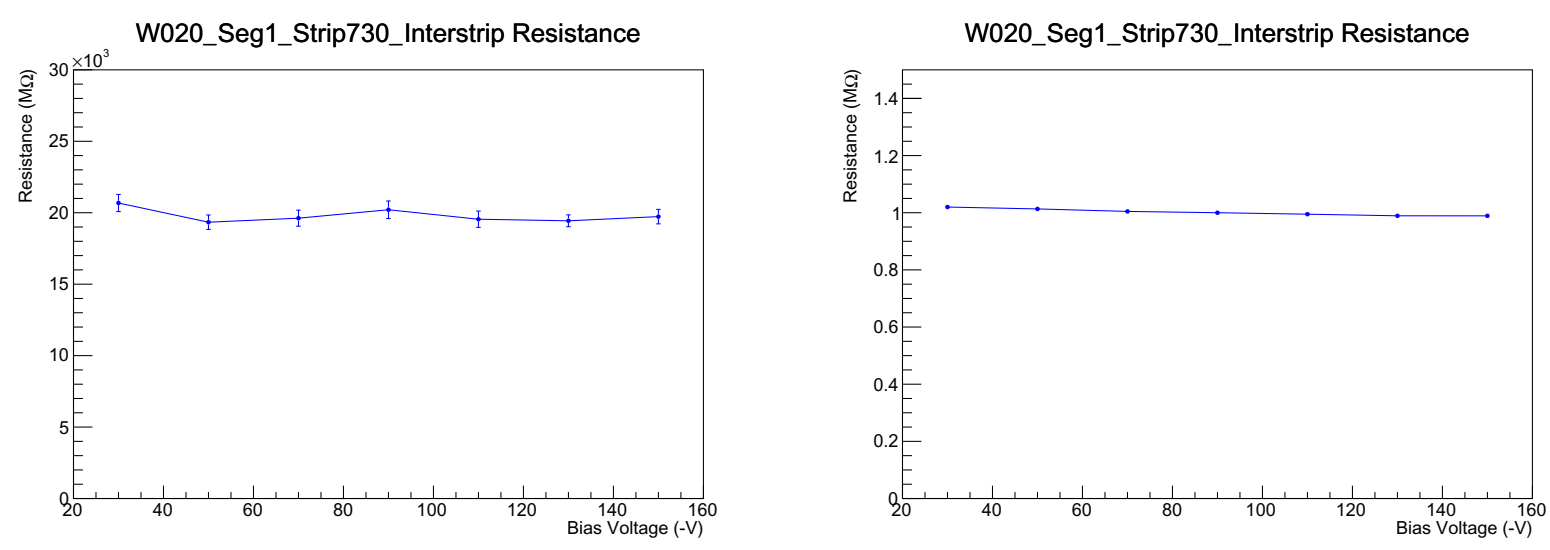

Figure 5.6: Measurements of the interstrip resistance at two different locations in segment 1 of sensor W020. (Left) A well behaved region and (right) a region with loss of isolation. Note the discrepancy in the vertical axis between the two plots. Error bars in the right graph are too small to display. The error is small in the case of low isolation because larger voltages are induced in the central strip.

\subsection{Properties of the Surface Oxide}

The surface oxide and the $\mathrm{Si}-\mathrm{SiO}_{2}$ interface are the focus of this final section. In particular, the metaloxide-semiconductor (MOS) capacitor is presented as a means to characterize two features of the surface oxide: oxide thickness, and surface charge density. First, a general description of MOS capacitors is given. This is followed by results from 21 total MOS capacitors which are a new inclusion in the suite of test structures for the pre-production run of ATLAS18 sensors. Finally, it is demonstrated that the oxide can be injected with or depleted of charge through long-term biasing of the MOS device.

\subsubsection{Characteristics of MOS Capacitors}

It is appropriate to describe the general characteristics of MOS capacitors in order to contextualize their utility in monitoring ITk sensor production. As the name suggests, the MOS capacitor is formed by layering a semiconductor substrate with an insulating oxide and then situating a sheet of metal (called a gate) above the oxide. Typically, the semiconductor is held at ground while a voltage is applied to the gate. The capacitance of this structure will depend on how the semiconductor responds to different potentials applied to the gate.

Take p-type silicon as the semiconductor of a MOS device ${ }^{2}$. With the application of some large (it will soon become apparent what is meant by "large") negative voltage at the gate, a potential difference will also be induced across the semiconductor causing positive holes to accumulate at the silicon-oxide interface. In this state of accumulation the MOS device acts like a parallel plate capacitor where the capacitance depends

\footnotetext{
${ }^{2}$ It is instructive to focus on one type for clarity although the same arguments are true for n-type semiconductors as well. The important distinction is in the sign of the charge carriers which will lead to a relative flip of the CV in the voltage axis between n-type and p-type.
} 
on the area of the gate and the distance between the gate and the silicon-oxide interface, that is, the thickness of the oxide. The measured capacitance is called the oxide capacitance and is given by

$$
C_{o x}=\frac{\epsilon_{o x} A}{t_{o x}}
$$

where $\epsilon_{o x}$ is the permittivity of the oxide, $A$ is the gate area, and $t_{o x}$ is the oxide thickness.

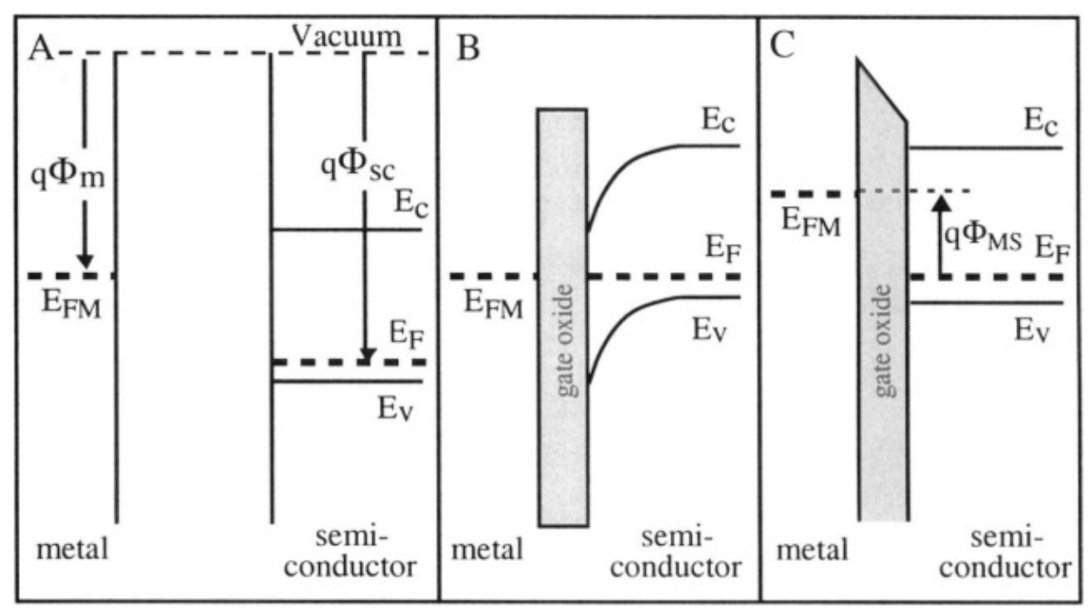

Figure 5.7: Energy band representation of the flatband condition. (A) The materials on their own with different fermi levels and associated work functions. (B) The materials in a MOS capacitor with no voltage applied to the gate. The energy bands of the semiconductor bend down. (C) The band diagram of the materials when $V_{F B}$ is applied to the gate and the flatband is restored in the semi-conductor. [16]

Increasing the voltage will eventually result in a state where positive holes no longer pile up near the interface. This is known as the flatband condition and is best understood through examination of the energy bands in the material (see Figure 5.7). On their own, the fermi levels of the metal and the semiconductor are not equal. Alignment of the fermi levels occurs when the metal and silicon are brought near to each other in the MOS device. The associated charge transfer in this process bends the energy bands of the silicon downwards. In order to restore the flatband condition in the silicon, a gate voltage equal to the difference in the work functions of the materials must be applied. This is called the flatband voltage and is defined as

$$
V_{F B}=\Phi_{M}-\Phi_{S} \equiv \Phi_{M S}
$$

where $\Phi_{M}$ and $\Phi_{S}$ are the work functions of the metal and silicon, respectively. The flatband capacitance can also be defined as ${ }^{3}$

$$
\frac{1}{C_{F B}}=\frac{1}{C_{o x}}+\frac{\lambda_{d}}{A \epsilon_{S i}} ; \quad \lambda_{d}=\sqrt{\frac{\epsilon_{S i} k T}{q^{2} N_{A}}}
$$

\footnotetext{
${ }^{3}$ This definition is essentially taken for granted here. The flatband capacitance consists of the oxide capacitance plus a series capacitance contribution from the silicon.
} 
where $\lambda_{d}$ is the Debye length, $\epsilon_{S i}$ the permittivity of silicon, $k$ the Boltzmann constant, $T$ the temperature of silicon, $q$ the elementary charge, and $N_{A}$ the doping concentration of the p-type silicon.

Increasing the potential applied to the gate beyond the flatband voltage will cause the silicon to begin depleting as a step junction (section 2.3). The series capacitance contribution of the silicon will decrease the total capacitance of the MOS device as the depletion width grows. This will continue until a threshold when significant conduction of the minority carriers (electrons for p-type silicon) occurs. This is called inversion and corresponds to bending the conduction band of the silicon downwards (as in Figure 5.7-B). The total capacitance of the MOS device eventually returns to $C_{o x}$ with strong inversion.

A sheet charge at the interface of the silicon and oxide will result in a total shift of the flatband voltage, and indeed, any well-defined point of the CV curve. This leads to a correction of equation 5.2

$$
V_{F B}=\Phi_{M S}-\frac{q A N_{o x}}{C_{o x}}
$$

where $N_{o x}$ is the surface charge density. [15],[16],[32]

\subsubsection{MOS Capacitors of ATLAS18R0 Sensors}

As mentioned in the preamble of this section, MOS capacitors have been newly added to the suite of QA test structures implemented on the half-moons of the pre-production ATLAS18 sensors. Measurement of the ATLAS18 MOS capacitors will allow QA sites to monitor the consistency of sensor processing, specifically the surface oxide. For QA purposes, the parameters of interest are the oxide capacitance, oxide thickness, flatband voltage, and surface charge density. Being newly implemented test structures, the collaboration has yet to establish a rigid test procedure or acceptance criteria. Here, a suggested procedure is outlined and results given from testing 21 total MOS devices.

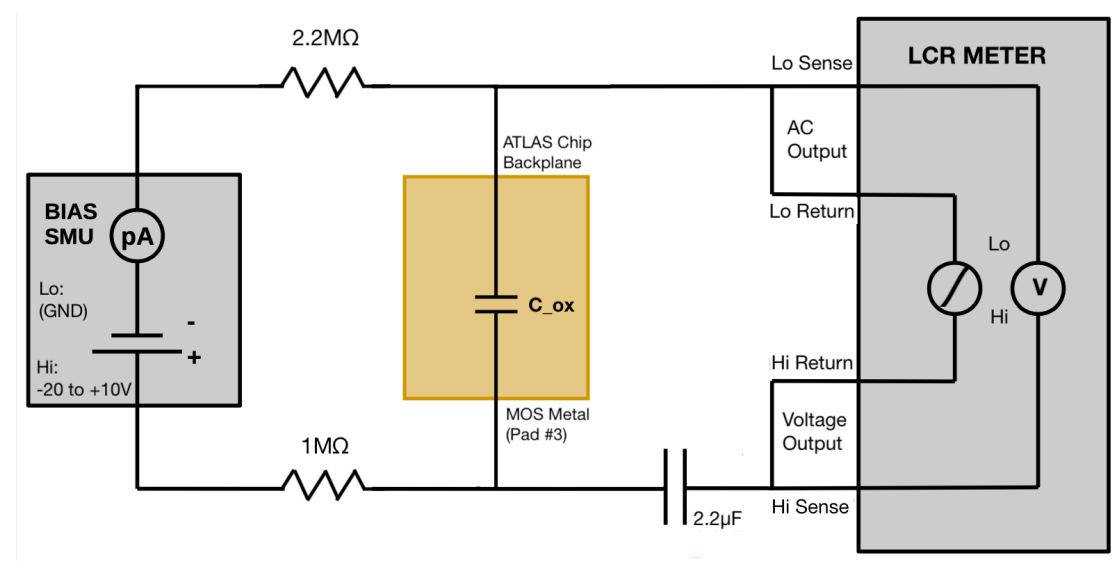

Figure 5.8: Circuit diagram of the test setup used to obtain the CV of the ATLAS18 MOS capacitor.

The test circuit for obtaining the MOS CV is shown in Figure 5.8. The setup requires an LCR meter 
with sub-picofarad precision and a biasing SMU. The AC output of the LCR meter and SMU ground (low output) are connected to the half-moon backplane. The voltage output of the LCR meter and high output of the SMU are connected to the contact pad for the MOS gate. The $2.2 \mu \mathrm{F}$ capacitor is used to protect the LCR meter from any large current spikes, for instance, in case the oxide breaks down. The resistors in line with the SMU outputs act as current limiters and help reduce any noise induced in the LCR meter. The capacitance is measured by the LCR meter sourcing a $10 \mathrm{kHz}, 100 \mathrm{mV}$ AC signal as the gate potential is varied from $+2 \mathrm{~V}$ to $-20 \mathrm{~V}$ in $0.1 \mathrm{~V}$ steps.

Figure 5.9 shows the CVs of 21 MOS capacitors along with four histograms of the parameters of interest for QA. The top right image gives a reference for the locations of each half-moon on the wafer as labelled in the plot legends. The oxide capacitance is taken as the capacitance measured at a gate voltage of - $20 \mathrm{~V}$. This is then used according to equations 5.1 and 5.3 to calculate the oxide thickness and flatband capacitances, respectively. The flatband voltage is taken as the linearly interpolated value where the $\mathrm{CV}$ intercepts the flatband capacitance on the accumulation side of the curve. With the flatband voltage and oxide capacitance determined, the oxide surface charge density is calculated using equation 5.4.

Overall, each of the four distributions are qualitatively normal with almost all of the data falling within 2.5 standard deviations of the mean. There is only one exception in the distribution of flatband voltages. Less than $10 \%$ total variation in the oxide capacitance and thickness is observed which indicates consistency in the oxide processing.

\subsubsection{Field Enhanced Charge Injection}

Besides the scope of the parameters monitored for QA, the MOS devices can be used to study the effects that long term biasing has on the surface charge density of the oxide. The measurement consists of taking multiple CVs of a single MOS device in succession. The first CV is taken in the forward direction, that is, scanning the gate voltage from inversion to accumulation. Next, the $\mathrm{CV}$ is taken by scanning in the reverse direction, accumulation to inversion. The device is then held at a constant positive bias for some time, $t_{b i a s}$, and the CV taken again in the forward direction. Lastly, the device is held at constant negative bias for the same $t_{\text {bias }}$ and the reverse scan performed. This process is repeated for various values of $t_{\text {bias }}$. Translations of the CVs in the voltage axis correspond to changes in the oxide charge density.

The phenomenon of field enhanced charge injection has been observed for three electric field strengths on time scales of $<2$ hours (Figure 5.10). The change in surface charge density is plotted against bias time. For each of the three field strengths shown, the initial surface charge density, $N_{o x}^{0}$, is calculated from the first forward scan CV curve. The $N_{o x}$ is calculated from each consecutive curve and then $\Delta N_{o x}=N_{o x}-N_{o x}^{0}$. The electric field strength under bias is calculated according to

$$
E \approx \frac{\left|V_{g a t e}-V_{F B}\right|}{t_{o x}}
$$

where $V_{\text {gate }}$ is the potential the gate is held at between scans. 



Figure 5.9: MOS Capacitors. 
In the case of the smallest field $(0.22 \mathrm{MV} / \mathrm{cm})$ during positive bias (inversion), electrons are discharged from the oxide to the metal gate faster than they are injected into the oxide from the silicon. Under negative bias (accumulation), the negative charges in the oxide move toward the interface and decrease the total charge density. This negative bias effect saturates around one hour with no additional change seen after two hours of bias. In the intermediate field $(0.50 \mathrm{MV} / \mathrm{cm})$ the charges moving to and away from the interface are balanced and only a net difference between forward and reverse directions is observed. Finally, for a large field $(1.00 \mathrm{MV} / \mathrm{cm})$ in the positive bias case, the strong inversion allows for the electrons to be injected into the oxide. This injection happens faster (or more strongly) than the discharging of electrons and injection of positive charges during accumulation.

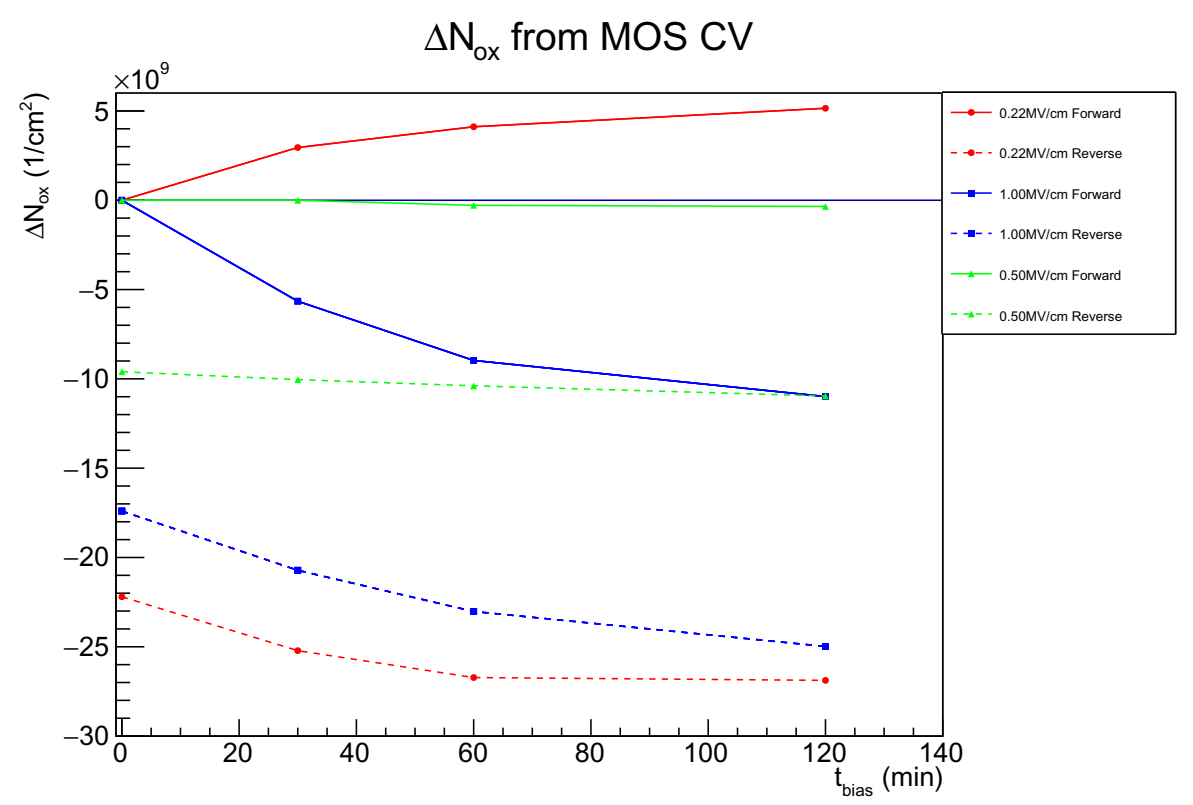

Figure 5.10: Field enhanced charge injection of a MOS capacitor for three field strengths. 


\section{Chapter 6}

\section{Summary and Outlook}

\subsection{Summary}

This thesis has presented a broad description of the HL-LHC upgrade and the need for the ITk to be installed in the ATLAS detector. The deficiencies of the ID, namely its inability to withstand the instantaneous luminosities expected at the HL-LHC, were outlined with the intention of motivating its replacement by the ITk. Details of the ITk layout and expected performance were also given. A brief excursion into semiconductor theory provided the background for a better understanding of the principle of operation of silicon strip sensors and their bulk properties.

The ITk strip sensors are the focus of this document. The long list of sensor specifications, both physical and electrical, reveal the need for QC testing. The suite of QC tests as described provide a way for the ITk strip sensor collaboration to verify these sensor specifications. Especially during manufacturing of preproduction and production generation sensors, it is critical that institutions tasked with QC are prepared. Establishing the readiness of the Carleton site to effectively carry out QC is partly the goal of chapters 3 and 4. This is crucial as the first pre-production sensors have been manufactured and await QC validation.

The effects of radiation on leakage current put forward in chapter 5 provide evidence that the expectations of sensor performance after 10 years of HL-LHC operation are reasonable. An example of a sensor experiencing loss of strip isolation demonstrates an aspect of sensor design which requires more attention. Finally, the MOS device was introduced as a means to monitor the processing of the oxide and the topic of field enhanced charge injection was briefly explored.

It has been confirmed that the QC procedures developed by the ITk collaboration are effective in characterizing both physical and electrical sensor properties. A great deal of work has been done to develop test setups at the Carleton site. The image capture and metrology systems were completely established from non-existent to working setups throughout the duration of this Master's work. Similarly, this work also resulted in the crucial development of the full strip test as implemented with the semi-automatic probe station and switch matrix. As the ITk project moves forward, Carleton is ready to serve as a QC site during sensor production. 


\subsection{Outlook}

Along with the success in developing Carleton as a full-fledged QC site and the work done to validate the ITk sensor design there are areas requiring further attention. These areas are detailed below. In all cases, action is being taken to rectify these situations and to better understand and classify the more exceptional sensor properties.

Carleton is being further streamlined to have a total throughput of up to 20 sensors per week. This includes semi-automated acquisition of the IV and CV tests in a dry environment. The setup involves a customized low humidity storage container which can hold 20 sensors at once each in individual jigs. A software controlled switch matrix will be used to sequentially connect each sensor to the test circuit and perform the IV and CV tests.

Campaigns to irradiate pre-production sensors are underway. This is perhaps the most effective way to experimentally predict what the ITk detector performance will look like at the end of lifetime. Carleton is poised to participate in these studies of irradiated sensors employing the same cold jig presented in chapter 5. This jig was designed with the testing of main sensors in mind and would allow any of the QC parameters to be measured in the irradiated sensors.

A humidity sensitivity has been observed in the prototype sensors. Only a dependence of the breakdown voltage and leakage current have been extensively tested. The Carleton site is prepared to continue studying this humidity sensitivity with the recent development of humidity controlled test environments. This will allow any of the QC tests to be performed at a broad range of relative humidities ( $0 \%$ up to $90 \%$ ). Additionally, any of the QA test structures can also be subjected to the same scrutiny.

The use of the MOS device to study the oxide marks the beginning of efforts in the strip community to better understand surface effects in the ITk sensors. The initial tests of these devices demonstrate that the phenomenon of charge injection is present. The gated diode structures also appear promising for the revelation of surface current contributions and the sheet resistance at the oxide interface. The characterizations of the surface oxide and silicon-oxide interface will prove valuable to inform sensor models, which have not included these effects.

As alluded to in chapter 5, there are anomalous cases where strip isolation is lost across large regions of sensors. The origin of this issue is not well defined although there has been some success in letting problematic sensors "rest" for extended periods of time in dry storage. A publication describing these anomalous cases has resulted but a better understanding of the surface effects and humidity sensitivity seem most likely to enlighten this situation. 


\section{Bibliography}

[1] Mobs E. "The CERN accelerator complex Complexe des accélérateurs du CERN"[photo]. 2016[updated 2017 Aug 11; accessed 2020 Feb 1]. https://cds.cern.ch/record/2197559

[2] HL-LHC Industry: Industry Relations and Procurement Website for the HL-LHC project. "Project Schedule". [accessed 2020 Feb 1]. https://project-hl-lhc-industry.web.cern.ch/content/projectschedule

[3] The ATLAS Collaboration. "The ATLAS Experiment at the CERN Large Hadron Collider". JINST 3 S08003; 2008.

[4] Klein CT. "Investigation of performance and the influence of environmental conditions on strip detectors for the ATLAS Inner Tracker Upgrade"[doctoral dissertation]. Cambridge, England: University of Cambridge; 2019.

[5] ATLAS Inner Detector Community. "Inner Detector Technical Design Report". Volume I. CERN/LHCC 97-16 / ATLAS TDR 4; 1997.

[6] The ATLAS Collaboration. "Technical Design Report for the ATLAS Inner Tracker Strip Detector". CERN-LHCC-2017-005 / ATLAS-TDR-025; 2017.

[7] The ATLAS Collaboration. "Measurement prospects for VBF $H \rightarrow W W^{(*)} \rightarrow e \nu \mu \nu$ production with $3 \mathrm{ab}^{-1}$ of HL-LHC pp-collisions”. CERN / ATL-PHYS-PUB-2016-018; 2016.

[8] Miyagawa PS, Dawson I. "Radiation background studies for the Phase II inner tracker upgrade". CERN / ATL-UPGRADE-PUB-2013-012; 2013.

[9] The ATLAS Collaboration. "Measurement of the Inelastic Proton-Proton Cross Section at $\sqrt{s}=$ $13 T e V$ with the ATLAS Detector at the LHC". CERN-EP-2016-140; 2016. arXiv:1606.02625v4 [hep-ex]

[10] Ferrari A, Sala PR, Fasso A, Ranft J. "FLUKA: A multi-particle transport code (Program version 2005)". CERN-2005-010 / SLAC-R-773 / INFN-TC-05-11; 2005. 
[11] Sjöstrand T, Mrenna S, Skands PZ. "A Brief Introduction to PYTIA 8.1”. Comput Phys Commun. 2008; Vol.178: p.852-867. arXiv:0710.3820 [hep-ph].

[12] The ATLAS Collaboration. "Performance of the ATLAS Silicon Pattern Recognition Algorithm in Data and Simulation at $\sqrt{s}=7 T e V "$. CERN / ATLAS-CONF-2010-072; 2010.

[13] Salzburger A. "Optimization of the ATLAS Track Reconstruction Software for Run-2". J Phys Conf Ser. 2015; Vol.664: 072042. doi: 10.1088/1742-6596/664/7/072042

[14] The ATLAS Collaboration. "A neural network clustering algorithm for hte ALTAS silicon pixel detector". JINST 9 P09009; 2014

[15] Colinge JP, Colinge CA. "Physics of Semiconductor Devices". Dordrecht (Netherlands): Kluwer Academic Publishers; 2002.

[16] Grove AS. "Physics and Technology of Semiconductor Devices". New York (NY): John Wiley and Sons, Inc.; 1967.

[17] Wikimedia Commons, TheNoise. "File:Pn-junction-equilibrium.png”[photo]. 2007[accessed 2020 Mar 1]. https://commons.wikimedia.org/wiki/File:Pn-junction-equilibrium.png

[18] THF Group.“Silicon Detector gif”[photo]. [accessed 2020 Mar 1].

[19] Terada S et al. "Proton irradiation on p-bulk silicon strip detectors using $12 \mathrm{GeV}$ PS at KEK". Nucl Instrum Methods Phy Res A. 1996; Vol.383: p.159-165. doi: https://doi.org/10.1016/S01689002(96)00748-6

[20] Hara K et al. "Performance of irradiated n+-on-p silicon microstrip sensors". Nucl Instrum Methods Phy Res A. 2006; Vol.565: p.538-542. doi: https://doi.org/10.1016/j.nima.2006.06.051

[21] Mikestikova M et al. "Electrical characterization of surface properties of the ATLAS17LS sensors after neutron, proton and gamma irradiation". Nucl Instrum Methods Phy Res A. 2020[available 2020 Jul 23]; Vol.983. doi: https://doi.org/10.1016/j.nima.2020.164456

[22] Mikestikova $M$ et al. "Study of surface properties of ATLAS12 strip sensors and their radiation resistance". Nucl Instrum Methods Phy Res A. 2016; Vol.831: p.197-206. doi: https://doi.org/10.1016/j.nima.2016.03.056

[23] Hunter RHF. "Development and Evaluation of Novel, Large Area, Radiation Hard Silicon Microstrip Sensors for the ATLAS ITk Experiment at the HL-LHC'[master's thesis]. Ottawa ON: Carleton University; 2017. Copyright permission obtained[multiple photos].

[24] Hommels LBA et al. "Detailed studies of full-size ATLAS12 sensors". Nucl Instrum Methods Phy Res A. 2016; Vol.831: p.167-173. doi: http://dx.doi.org/10.1016/j.nima.2016.03.042 
[25] Hunter RFH et al. "First bulk and surface results for the ATLAS ITk Strip stereo annulus sensors". Nucl Instrum Methods Phy Res A. 2019; Vol.924: p.142-146. doi: https://doi.org/10.1016/j.nima.2018.08.031

[26] Klein CT et al. "Initial tests of large format sensors for the ATLAS ITk strip sracker". Nucl Instrum Methods Phy Res A. 2021[available 2020 Sep 25]; Vol.986. doi: https://doi.org/10.1016/j.nima.2020.164677

[27] Unno $Y$ et al. "Development of n+-in-p large-area silicon microstrip sensors for very high radiation environments - ATLAS12 design and initial results". Nucl Instrum Methods Phy Res A. 2014; Vol.765: p.80-90. doi: http://dx.doi.org/10.1016/j.nima.2014.06.086

[28] Robinson D. “ATLAS QA Procedures for Silicon Microstrip Detectors”. 1st Workshop on QA Issues in Silicon Detectors. 2001.

[29] Wiik-Fuchs L et al. "Annealing studies of irradiated p-type sensors designed for the upgrade of ATLAS phase-II strip tracker". Nucl Instrum Methods Phy Res A. 2019; Vol.924: p.128-132. doi: https://doi.org/10.1016/j.nima.2018.10.014

[30] Helling C et al. "Strip sensor performance in prototype modules built for ATLAS ITk". Nucl Instrum Methods Phy Res A. 2020[available 2020 Jul 18]; Vol.978. doi: https://doi.org/10.1016/j.nima.2020.164402

[31] Fernández-Tejero J et al. "Humidity sensitivity of large area silicon sensors: Study and implications". Nucl Instrum Methods Phy Res A. 2020[available 2020 Jul 17]; Vol.978. doi: https://doi.org/10.1016/j.nima.2020.164406

[32] Mego TJ. "Guidelines for Interpreting CV Data”. Solid State Technology. Cleveland (OH). Keithley Instruments; 1990. 